\title{
Glossaries and Logograms with Cuneiform Signs
}

\section{Uncertain Readings and Words}

bappak (syllabic): NY 5'

GI.ZÚ.LUM.MA 'date reed plant' $\rightarrow z \bar{e} r /$ arti GI.ZÚ.LUM.MA

huhahi (syllabic): IGI 1: 70'

ITI.1.KAM 'one month': IGI 3: 36'

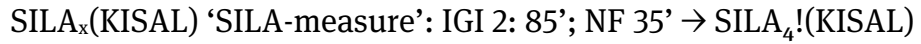

SILA $_{4}$ !(KISAL) 'SILA-measure': IGI 1: 8', 12', 29', 41' $\rightarrow$ SILA $_{x}\left(K_{I S A L}\right)$

ŠU.BI.AŠ.ÀM 'ditto': IGI 1: 158', 162'

ŠU.BI.GIN7.NAM 'ditto': IGI 1: 124', 131', 135', 149’, 156'; IGI 3: 84’

tamadraš (syllabic) 'magical spell': IGI 1: 147'

TU6.ÉN.É.NU.RU: IGI 1: 76'

UD.1.KAM 'first day': NZ 11'

UD.3.KAM 'third day': IGI 1: 9', 200'; IGI 2: 1, 6, 75', 78', 114'

UD.9.KAM 'nine days': IGI 1: 85'

UD.14.KAM 'fourteen days': NV 5'

UD.15.KAM 'fifteen days/fifteenth day': IGI 2: 114'

UD.20.KAM 'twenty days': IGI 1: 64'; NU 6'

UD.21.KAM 'twenty first day': IGI 3: 36'

upak (syllabic): NY 5, 6'

\section{Hurrian}

hu-tu-ul 'to glorify?': see notes to IGI 1: 182'

\section{Numbers}

$1 \rightarrow i s ̌ t e ̄ n$

1-niš $\rightarrow$ ištēniš

$1-\check{s} u$ $\rightarrow$ ištiššu

$2-m a \rightarrow$ šitta

$2-\check{u} u$ šinišu

2,30 or $150 \rightarrow$ šumēlu

$3 \rightarrow$ šalāš

3-šú $\rightarrow$ šalāšǐšu

$4 \rightarrow$ rebû

$5 \rightarrow$ hamiš, hamšat 
$6 \rightarrow$ šediš

$7 \rightarrow$ sebe

$8 \rightarrow$ samānat

$9 \rightarrow$ tiše, tišît

$10 \rightarrow$ ešeret

$11 \rightarrow i$ štēššeret

$14 \rightarrow$ erbēšeret

$15 \rightarrow$ imittu, hamiššer

$20 \rightarrow$ šarru, logogram MAN

$30 \rightarrow{ }^{\mathrm{d}} 30$, Sîn

$40 \rightarrow{ }^{\mathrm{d}} 40, E a$

150 or $2,30 \rightarrow$ šumēlu

\section{Dividing and Ditto Signs}

: 'a dividing sign': IGI 1: 34’, 35’, 47’, 135', 155’, 156', 158', 164’, 165’, 166', 167', 168', 169', 170', 171', 172'; IGI 2: 5, 20, 21, 70', 74', 101', 116', 141'; IGI 3: 37’, 38', 39’, 52' KIMIN $\rightarrow$ ašar šanîmma 'ditto'

MIN $\rightarrow$ šanîš ‘alternatively, ditto’

ŠU.BI.AŠ.ÀM (̌̌U, no. 567) $\rightarrow$ ŠU.BI.AŠ.ÀM in Uncertain Readings and Words ŠU.BI.GIN $7 . N A M(G I M$, no. 686) $\rightarrow$ ŠU.BI.GIN $7 . N A M$ in Uncertain Readings and Words

\section{Divine Names}

Adad ('IŠKUR) 'the weather god Adad': IGI 3: 62'

Anu (syllabic) 'the sky god Anu': IGI 1: 102', 120', 121', 134'

Asalluhi (syllabic) 'God of incantations, son of Ea': IGI 1: 94', 106', 191'; NF 36'

Dāmu (syllabic) 'god Dāmu': IGI 1: 95’, 106’; NF 40' $\rightarrow$ dāmu 'blood'

'LAMA.MEŠ $\rightarrow$ lamassatu 'pupil of the eye'

'ŠE.TIR $\rightarrow$ ašnan 'grain'

Ea (syllabic and d40) 'god Ea': IGI 1: 94', 106', 151'; IGI 3: 85’; NU 16'

Enki ( ${ }^{\mathrm{d} E n-k i) ~ ' g o d ~ E n k i ’: ~ N F ~ 37 ’, ~ 38 ' ~}$

Enlil ('En-líl) 'god Enlil'; NF 13'

Gula ('Gu-la) 'the goddess of healing, Gula': IGI 1: 75’, (76'), 95', 100', 106', 107', 191', 192'; IGI 2: 221'; NZb 1'

Ištar ('15, 'IŠs-tár, 'IŠ-tar) 'the goddess Ištar': IGI 3: 63', 66'; NF 14'; NV 7'

Mami 'the mother goddess Mami': IGI 1: 177',

Marduk ('AMAR.UTU) 'the king-god Marduk' IGI 1: 130', 151', 191'; NF 37'

Nabû ('AG) 'god Nabû': IGI 2: 217’; NF 40'

Nammu ('Engur) 'the goddess Nammu': IGI 1: 169', 173’ 
Nanāya ( $\mathrm{d}$ Na-na-a) 'the goddess Nanāya: NF 14'

Ningirimma ('Nin-girima) 'mistress of incantations': IGI 1: 95', 107', 191'

Ninlil ('Nin-líl) 'the goddess Ninlil, consort of Enlil': NF 13'

Ninurta ('Nin-urta) 'god Ninurta': IGI 2: 221'; NF 39'

Sîn ('30) 'the moon god': IGI 1: 197’, 198'

Šakkan ('GÌR) 'the god Šakkan': IGI 1: 93'

Šamaš ('UTU) ‘the sun god': IGI 1: 197’, 198'; IGI 3: 77’; NF 19', 28'; NS 4'; NZa 10' $\rightarrow$ șit šamši

Šazu ('šà-zu) 'the god Šazu, lit. the one who knows the inside, an incarnation of Marduk: NF 37', 38'

Šeriš ( $\left.{ }^{\mathrm{d}} \mathrm{GU}_{4}\right)$ 'the ox god' $\rightarrow$ kabūtu

Šulpaea (syllabic) 'god Šulpaea': IGI 3: 62'

Tašmētu (syllabic) 'goddess Tašmētu': IGI 2: 217’

\section{Month Names}

Nisannu (itiÁRA) 'month Nisannu': NZ 11'

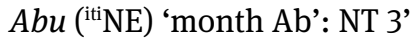

Tebētu (iti $\mathrm{AB})$ 'month Tebet': NT 3'

\section{Personal names}

Aššur-bāni-apli (' ${ }^{\mathrm{m}}$ š-šur-DÙ.A) ‘Ashurbanipal’: IGI 2: 217’

\section{Akkadian Words}

\section{Notes:}

- References are given even if reconstructed from broken bits.

- Materia Medica is printed in bold.

- $\quad$ The relevant parallels of the Nineveh IGI are not listed in the glossary.

- Mss. NI (V.1 UGU 1 = BAM 480) and NX (V.2 UGU 2 = BAM 482) are indexed by Worthington 2005; 2007, and Attia and Buisson 2003. The Mss. in chapter VI Diagnostic Medical Omens Concerned with Sick Eyes (Diagnostic Handbook, Sakikkû Chapter 5) will be indexed in the forthcoming publication of Eric Schmidtchen. Therefore, these texts are excluded from the present glossary, in order to omit unnecessary repetition.

- Akkadian follows $C D A$ and not $C A D$. 


\section{A}

$a b \bar{a} k u$ (syllabic) 'overturned, reversed'

G-stem: $a b-k u$ : NZb 6'

abālu (HÂD.DU) 'to dry'

G-stem: ablat(HÂD.DU): IGI 2: 71', 157'; itablu(HÁD.DU): IGI 2: 71', 157’

D-stem: tubbal(HÂD.DU): IGI 2: 74', 154', 168'; tu-bal: NZa 10'

abālu (syllabic) 'to stir ingredients' < wabālu

Št'ㄹ-stem: tuš-tab-bal: IGI 1: 33’

aban gabî (NA 4 ga-bi-i) 'alum': IGI 1: 35’; IGI 2: 8, 10, 57', 66'; NK 8'; NR 2', 7'

aban hurāṣ̦i (NA4 KUG.GI) 'golden bead'; NT 10'; NZb 4'

aban sābi (NA $\left.\mathrm{NA}_{4} \mathrm{~s} a-a-b i\right)$ 'sābu-stone': NZb 4'

aban tašrīti (NA $\left.{ }_{4} \mathrm{BAL}\right)$ 'tašrìtu-stone': IGI 1: 47'; IGI 3: 4

abāru (A.GAR $)$ 'lead': IGI 2: 198'; IGI 3: 95', 105' $\rightarrow$ irri / itqūr abāri

abnu ( $\left.\mathrm{NA}_{4}\right)$ 'stone, bead': IGI 3: 34', 37’ $\rightarrow$ aban hurāṣi/mūṣi/sābi; qēm aban suluppī abu (AD) 'father': IGI 1: 151'

$a b u \rightarrow$ Month Names

$a b \hat{u} \rightarrow a p \hat{u}$

abukkatu (LI.TAR/DUR) 'abukkatu-plant' $\rightarrow$ hìl abukkati

adappu (syllabic) 'canal': IGI 3: 25'

adi (EN) 'until’: IGI 2: 71', 119', 157', 220'; IGI 3: 54'

agalapû (syllabic) 'agalapû-lagoon': IGI 1: 92'

agašgû (A.GA.ÁŠ.GA) 'novice (physician)': IGI 1: 70'

agubbû (A.GÚB.BA.A) 'holy water vessel': IGI 3: 76'; NF 17'

ahātu (syllabic) 'sister': IGI 1: 101', 121', 126'

ahāzu (syllabic) 'to take, acquire'

G-stem: $e$-hu-uz-zu: IGI 2: 218', 219'

ahê (syllabic) 'by itself, separately': IGI 2: 161'

ahennû (syllabic) 'separately, individually': IGI 1: 82', 117', 143', 180'; IGI 2: 136'

ahû (syllabic and BAR) 'stranger': IGI 2: 12; 'non-canonical (material)': IGI 2: 220'

ai ( $a-a)$ 'not', vetitive particle: NZb 9', 10'

akalu (NINDA) 'loaf of bread': IGI 2: 67'; IGI 3: 78'

akālu (syllabic and $\mathrm{GU}_{7}$ ) 'to eat, consume, hurt, cause pain'

G-stem: e-kal: IGI 1: 74’; ik-kal-ki-na-ši: IGI 1: 113’; ikkalma(GU $\left(\mathrm{GU}_{7}-\mathrm{ma}\right)$ : IGI 2: 131’;

ikkal(GU $\left(\mathrm{GU}_{7}\right)$ : IGI 3: 82', 88'; ikkalšu(GU $\left.\mathrm{GU}_{7}-\mathrm{šu}\right)$ : NV 6'

aktam ('ak-tam and 'at-kám) 'aktam-plant': IGI 1: 18'; IGI 2: 125', 195'; IGI 3: 72'; NR 4';

NV 1'

alādu (Ù.TU) 'to give birth' < walādu

G-stem: ullad(Ù.TU): IGI 1: 67’; ūlid(Ù.TU): IGI 1: 195'; ūlidu(Ù.TU): IGI 2: 110', 148';

NR 5'; ul-du: NRa 3' $\rightarrow$ larsīni ša halli lahri ša šumēli ša māšì ūlidu, šizbi

musukkati ša zikara ūlidu

alāku (syllabic and DU) 'to go (+ ventive to come)' 
G-stem: al-ka-ni: IGI 1: 114'; ta-la-ka-ni: IGI 1: 115'; il-lak: IGI 1: 121'; illakma(DU-

ma): IGI 2: 12, 14 ; illak(DU-ak): IGI 2: 22, 121’ $\rightarrow$ ālik mahri

alallû (syllabic) 'water pipe': IGI 1: 194'

alapû (syllabic) 'alapû-algae’: IGI 1: 92', 188'

alātu (syllabic) 'to swallow'

D-stem: ú-al-lat': IGI 1: 27'

ālik mahri (syllabic) 'the one who walks in front = a predecessor': IGI 2: 219'

ăliku (syllabic and DU(-ka/ka $\left.a_{16}\right)$ 'moving, loose tissue in the eyes': IGI 2: 19, 22, 26

alluharu (IM.SAHAR.BABBAR.KUR.RA) 'alluharu-mineral': IGI 1: 82'

alpu (GU $\left.{ }_{4}\right)$ 'ox' $\rightarrow$ kabūt/lipi/lipi kalīti alpi; dām kurșipti alpi șalmi; kalìt alpi șalmi

- $a(m)$, - ni(m) (syllabic) ventive suffix: IGI 1: 74', 103', 114', 115', 122', 123’, 163’, 187’,

189', 190'

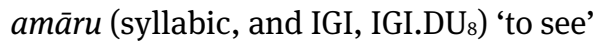

G-stem: i-mur-ma: IGI 1: 169'; immar(IGI.DU $\left.{ }_{8}\right)$ IGI 3: 73’, 74’; a-mur: IGI 3: 86’;

i-mar-ma: NF 20'; e-mur-šú-ma: NZb 7'; ìmaru(IGI.DU 8 ): NZa 10'

Gtn-stem: ìtanammaru(IGI-ru): NF 13'; ìtanammar(IGI(.IGI)-mar): NT 4'

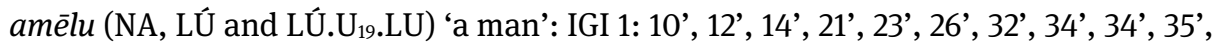

36', 40', 45', 54', 57', 59', 61', 71', 77', 79', 87', 88', 163', 166', 168', 169', 172', 173',

174', 184', 200', 201'; IGI 2: 1, 9, 12, 16, 17, 19, 22, 25, 27, 53', 54', 56', 58', 60', 61',

63', 76', 115', 116', 117', 121', 124', 126', 137', 139', 140', 141', 196', 199', 200', 202',

204', 207', 209', 213', 215', 216'; IGI 3: 1, 9, 10, 49', 51', 68', 70', 73', 74', 75', 77', 81';

NF 13', 17', 20', 23', 26', 32'; NK 4’, 6', 9'; NQ 5’, 8', 10'; NR 3’, 5’, 6’, 10'; NS 1', 6’; NT

4’; NV 1', 6'; NZ 19'; NZa 8' < awīlu $\rightarrow$ kalli gulgul amēli

amēlūtu (syllabic and NAM.LÚ.U ${ }_{19} . \mathrm{LU}$ ) 'human' $\rightarrow$, eșemti/šīnāti amēlūti

$\operatorname{ammin}(i)$ (syllabic) 'why': IGI 1: 112', 113', 178', 187’, 189'

\section{amurdinnu $\rightarrow$ murdinnu}

amurriqānu (IGI.SIG. $\mathrm{SIG}_{7}$ ) 'jaundice’: IGI 2: 115', 116' < awurriqānu

amurru (IM.4) 'west wind': IGI 1: 115'

ana (DIŠ) 'to, for, on, onto (also in compound prep.)': IGI 1: 8', 12', 102', 121', 151', 194';

IGI 2: 12, 14, 22, 106', 134'; IGI 3: 79', 80'; 'in, into, within': IGI 1: 3’, 80', 165', 185',

198'; IGI 2: 84', 156'; IGI 3: 34', 35', 53'; NY 7a'; 'for' a specific purpose: IGI 2: 222';

introducing a final sentence, 'in order to': IGI 2: 19, 22, 98', 124', 137’, 193'; IGI 3: 3,

11, NF 17', 20', 23'; NQ 11'; NS 1'; NU 12'; NV 7' $\rightarrow$ ana ašri šanîmma; ana imitti; ana

lèt; ana libbi; ana muhhi

ana ašri šanîmma (DIŠ KIMIN) 'alternatively’: IGI 1: 55’, 56’, 86’; IGI 2: 52’, 65’, 67’-74’,

208', 212'; IGI 3: 7, 23', 37'

ana imitti (ana ZAG) 'on, to the right': NF 15'

ana lèt (syllabic) 'to the side of someone': IGI 1: 121'

ana libbi (ana lìb-bi/ŠÀ) 'i/onto': IGI 1: 7’, 18', 26', 42', 48', 50', 56', 72', 81, 83', 84', 86',

87’, 88', 76', 78', 104'; IGI 2: 76', 78', 89', 115', 132', 145', 148', 158', 169', 175', 194';

IGI 3: 30', 71', 95'; NF 17', 21'

ana muhhi (ana UGU) 'onto, over': IGI 2: 4, 6, 52', 134' 
ana pān (ana IGI) 'in front of': IGI 1: 177'; IGI 3: 28', 77'

anāku (syllabic) 'I, me': IGI 1: 100', 152', 192'; NF 14'

annakku ( ${ }^{\text {na4 }}$ AN.NA) 'tin': IGI 3: 3; NF 10'

annânam (syllabic) 'this here; from now on': IGI 3: 36'

annitu (syllabic) 'this': IGI 1: 94'; IGI 3: 87'

annû (syllabic and ŠEŠ) 'this, these': IGI 1: 62', 64', 85’, 97’, 109'; IGI 2: 10, 11, 26; IGI 3:

5, 29', 34', 43'; NF 11'; NS 3'; NU 14'

annuharu (syllabic) 'annuharu-mineral': NZ 23'

anzahhu ( $\left.{ }^{\mathrm{na} 4} \mathrm{AN} . \mathrm{ZAH}\right)$ 'anzahhu-glass (bead)': NT 8'

apsû (syllabic) 'underground water': IGI 1: 171'

aptu (syllabic) 'window': NF 29'

ap/bû (syllabic) ‘cloudy, veiled (said of eyes)': IGI 1: 90', 99', 111', 123', 138', 176'; IGI 3:

26'; NK 9'; NQ 5', 10'; NS 1'

$a p / b \hat{u}$ (syllabic) 'to become cloudy, veiled (said of eyes)'

G-stem: tab-ba-a: IGI 1: 112'; ip-pa- $a$ : IGI 2: 54'

arādu (syllabic) 'go down, descend, plunge' < warādu

G-stem: ú-ri-du: IGI 1: 104'; ur-da: IGI 1: 194'

arāmu (syllabic) 'to cover'

G-stem: ár-mat: IGI 2: 121', 124'

arāpu $\rightarrow$ erēpu

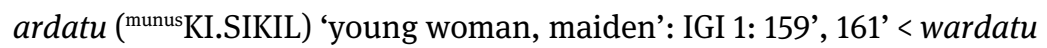

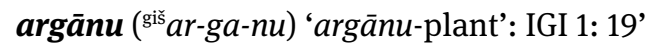

arhu (ITI) 'month': IGI 2: 138', 144'

āribu (UGA ${ }^{\text {mušen }}$ 'raven': IGI 2: 106' $\rightarrow$ pel āribi

arkabu (ARGAB ${ }^{\text {mušen }}$ ' 'bat' $\rightarrow$ rikibti arkabi

arki (EGIR) 'after(wards)': IGI 2: 53', 79'

arkǐšu (EGIR-šú) 'afterwards': IGI 3: 30', 54'

arkìtu (EGIR-tì) 'afterwards' $\rightarrow$ ina arkīti

arku (GID.DA) 'long (of sheep's bones)': IGI 2: 53'

arqu (SIG) 'yellow-green': IGI 1: 28' $\rightarrow$ mușa' 'irānu

arqūtu (SIG $\left.{ }_{7}-\mathrm{Su}\right)$ 'freshness', as qualification for 'fresh-green' plants (arqūssu, lit.

'concerning his freshness'): IGI 1: 10', 21', 80'; IGI 2: 63', 153’; NU 7'

arti bīni (PA giš bi-ni) 'leaf of tamarisk': IGI 2: 144'

arti ēri (PA gišsMA.NU) 'leaf of èru-tree'; IGI 2: 144'

arti GI.ZÚ.LUM.MA (PA GI.ZÚ.LUM.MA) 'leaf of date reed’: IGI 3: 71'

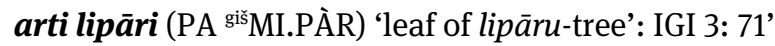

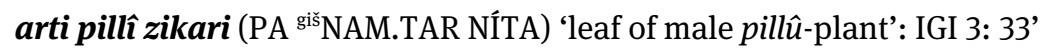

arti šunê (PA gišŠE.NÚ.A) 'leaf of šunû-tree': IGI 3. 70'

arti titti (PA giš PĚŠ) 'fig leaf': IGI 3: 70'

artu (PA) 'foliage, leafs' $\rightarrow$ arti bīni/êri/GI.ZÚ.LUM.MA/pillî zikari/šunê/titti

aru (syllabic) 'stalk, chaff': IGI 1: 114' 
ashar ( ${ }^{\text {na4 } a s ̌-h a r, ~}{ }^{\text {na4}}{ }^{a} a \check{s}$-har, presumably not ŠIM.BI.SIG SIG $_{7} \rightarrow$ dāmātu) 'ashar-stone':

IGI 1: 11', 13', 38', 58', 60', 72'; IGI 2: 21; IGI 3: 5, 104'; NR 2', 4'

askuppu $\left(\mathrm{KUN}_{4}\right)$ 'threshold': IGI 2: 28, 59', 103'

asu ( ̌̌m GÍR) 'asu-aromatic' $\rightarrow$ šaman asi

asû (lúA.ZU) 'physician’: IGI 3: 105’; NF 42’ $\rightarrow$ qātu

asûtu (A.ZU-ti) 'medical skill': IGI 1: 192'

așu (syllabic and È-a) 'to go, come out' < wașû

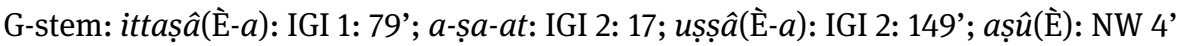

Gt-stem: lit-ta-și: IGI 1: 174'

Š-stem: ana ... šu-și-i: IGI 2: 98'; tušeșșēma(È-ma): IGI 1: 39’; tušeșși(È): NU 9'

ašāgu ( $\left.{ }^{g i s ̌ K I S ̌ I}{ }_{16}\right)$ 'ašāgu-acacia': NF 35' $\rightarrow$ šuruš ašāgi

ašar šanîmma (KIMIN) 'ditto’: IGI 1: 55’, 56’, 86’; IGI 2: 13, 15, 152’; IGI 3: 38’, 39’, 40’; NF

5', 6', 33'; NS 5', 7'; NU 7'; NZ 6', 7' $\rightarrow$ ana ašri šanîmma

ašgikû ( ${ }^{\mathrm{na} 4}{ }^{\mathrm{A}} \mathrm{Š}_{\text {.GI }}$. $\left.\mathrm{GI}_{4}\right)$ 'ašgikû-stone': NT 7'

ašitu (syllabic) 'blurring (of the vision)': IGI 1: 80'

aškāpu (' ${ }^{\text {lu }} \mathrm{AŠG} A B$ ) 'leatherworker’ $\rightarrow$ kammu ša aškāpi; šuhtu ša aškāpi

ašlu (syllabic) 'rush' $\rightarrow$ zēr ašli zikari

ašnan ('ŠE.TIR) 'grain' $\rightarrow$ qēm ašnan

aššum (syllabic) 'because of': IGI 1: 94'

Aššur (AN.ŠÁR ${ }^{\mathrm{ki}}$ ) 'Assyria': IGI 2: 217'

ašû (syllabic) 'blurred said of eyes (vision)': IGI 1: 90', 99', 111', 123', 137’, 167', 176'; IGI

2: 17; IGI 3: 26’; NK 9'; NQ 5', 10’; NS 1'; NY 3'

ašû (syllabic) 'to be blurred said of eyes (vision)'

G-stem: taš-šá-a: IGI 1: 112'; $a$-šâ- $a$ : IGI 1: 178'

$a$ šû (syllabic and MAŠ.TAB.BA) 'ašû-disease'; NY 4'

ašūhu (gišÙ.SUH ${ }_{5}$ ) 'ašūhu-pine’: NF 18', 21'

atā'išu ('KUR.KUR) 'atā'išu-plant': IGI 1: 32', 62'; IGI 2: 66', 189': IGI 3: 97’, 99', 102'; NR

6’; NU 10'

attā (syllabic) 'you' (2 m. sg. pron.): IGI 1: 130', 152'

attī (syllabic) 'you' (2 f. sg. pron.): IGI 1: 76'

attina (syllabic) 'you' (2 f. pl. pron.): IGI 1: 187'

awìlu $\rightarrow$ amēlu

awurriqānu $\rightarrow$ amurriqānu

ayabba (A.AB.BA) 'sea': IGI 1: 103'

aуyu (syllabic) 'which': IGI 1: 128', 129'

azugallūtu (syllabic) 'the medical knowledge/art': IGI 2: 221'

\section{B}

ba'ālu (syllabic) 'to be large'

G-stem: IGI 1: 67’ 
bābu (KÁ) 'entrance-door’: IGI 2: 12, 14

bakû (syllabic) 'to weep'

G-stem: i-bak-ki: IGI 1: 168'; i-bak-ka: IGI 1: 177’

balālu (HE.HE) 'to mix'

G-stem: taballal(HE.HE): IGI 1: 11', 13', 28', 40', 46’, 57', 63’; IGI 2: 6, 18, 24, 53', 76', 78', 92', 94', 113', 120', 123', 125', 146', 150', 152', 154', 155', 165', 192', 193', 194',

206', 215'; IGI 3: 1, 48', 53', 69', 72', 83'; NF 20', 23'; NK 12'

balāṣu $\rightarrow$ balșu

balāțu (syllabic and TI(.LA)) 'life’: IGI 1: 65’, 66’, 75’

balātu (syllabic and TI(.LA)) 'to live, heal, recover'

G-stem: ba-laț: IGI 1: 76'; i-bal-lu-uṭ: IGI 1: 161'; iballuț(TI): IGI 1: 13’; IGI 2: 99', 123', 125', 136', 138': IGI 3: 43’, 61', 65’, 67’, 72'; NF 14'; NU 5’, 6’; NV 3’; NZ 10'

D-stem: bullițīma(TI.(LA)-ma): IGI 1: 95', 107'; ú-bal-liț: IGI 1: 159' ; ana bulluțišu(TI-

šú) 'in order to heal it': IGI 2: 19, 22, 55', 137' IGI 3: 3; NQ 11'; NS 1'; (ana bulluṭi) NU

8', 12'; NV 7'; ana ... bul-lu-ți: IGI 2: 98'; bul-li-ți-ma: NZb 1'

ballukku (šmBAL / šmMUG) 'ballukku-aromatic': IGI 2: 69', 109'; NK 16’ $\rightarrow$ šaman

ballukki

balșu (syllabic) 'staring': IGI 3: 80', 86'

baltu ša țābti (syllabic) 'salted baltu-(thorn) plant': IGI 2: 112'

balțu (syllabic and TI.LA) 'healthy’: IGI 1: 97', 118', 181'; 'freshly’: IGI 2: 68'

balû $\rightarrow$ belû

baluhhu (šimBULUH) 'baluhhu-aromatic': NZ 9'; 'baluhhu-granule (in the eye)': IGI 1: 79'

$\rightarrow$ hì baluhhi

bāmâ (syllabic) 'in half': IGI 1: 70'

bāqilu (SAR.MUNU6; for MUNU6.SAR) 'malster' $\rightarrow$ mê bāqili

barāru (syllabic) 'to flicker (said of eyes)'

G-stem: bar-ra: IGI 2: 58'; i-bar-ru-ra: NZ 8'

barīrātu (syllabic) 'barīātu-sagapenum': IGI 1: 19'

barû (syllabic and IGI.KÁR) 'to collate (a tablet); to check (a remedy)'

G-stem: abrēma(IGI.KÁR-ma): IGI 2: 221'; ba-ri: IGI 3: 96’

bāṣu (syllabic) 'sand': IGI 1: 113'

bašālu (ك̌EG $6(-\check{a} a l))$ 'to be cooked'

Š-stem: tušabšal(ŠEG6(-šal)) ‘to cook, boil’: IGI 1: 18', 35’; IGI 3: 100’; NQ 8' bašāmu (syllabic) 'to create'

G-stem: ba-ša-mu: IGI 1: 194’; ba-áš-mu: IGI 2: 221’

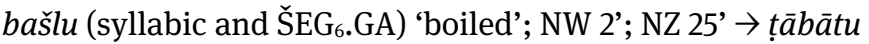

bâtu (syllabic) 'spend the night (under a star)'

Š-stem: tuš-bat: IGI 1: 81'; IGI 3: 34'; NF 19', 22'; NU 7'

bēltu (EN) 'mistress': IGI 1: 95', 107', 191', 192'

belû (syllabic) 'to end, terminate'

G-stem: li-be-la-a: IGI 1: 123’

beri/u- $\rightarrow$ ina beri/u- 
berqu (NIM.GÍR) 'lighting’: IGI 3: 9

billatu (syllabic and DIDA) 'beer mash': IGI 2: 168'; NZ 15'; NZ 18'

bīnu (syllabic and siššINIG) 'tamarisk': IGI 1: 80'; IGI 2: 5; IGI 3: 23', 51'; NF 17', 21' $\rightarrow$ arti/dām/mê/zēr bīni

biri $\rightarrow$ beri

birki immeri (bir-ki UDU) 'sheep testicle (lit. 'knee')': IGI 1: 185'

bi/urșu (syllabic) 'flash of light': IGI 3: 2; NF 13', 17', 20', 23'; NT 4'

bitu (É) 'house': IGI 2: 12, 14; NF 19'; 'domestic': NS 4'

bulālu (syllabic) 'bulālu-plant': IGI 3: 37'

bultu (syllabic and TI) 'cure, remedy, medical prescription': IGI 1: 76’; IGI 2: 220’

buqlu $\left(\mathrm{MUNU}_{6}\right)$ 'malt' $\rightarrow$ qeem buqli

burāšu ('̌imLI) ‘juniper’: IGI 2: 5, 16; IGI 3: 11, 55’, 77’, 93’; NF 34’; NU 3’; NV 4'; NZ 9' $\rightarrow$ $q \bar{e} \bar{m} / z \bar{e} r$ burāši

burșu $\rightarrow$ birșu

būrtu (PÚ) 'well' $\rightarrow$ mê būrti

buṣinnu (syllabic) 'a wick’: IGI 2: 90'

\section{D}

dagālu (syllabic) 'to see, look'

Gtn-stem: id-da-nag-ga-la: IGI 3: 2

dalāhu $\rightarrow$ dalhu

dalhu (syllabic and LÙ.LÙ) ‘troubled': IGI 1: 123', 166’, 184'; IGI 2: 215’; IGI 3: 1; NR 6' daltu (gišIG) 'door': IGI 3: 88'

dām bīni (MÚD gišsi-ni) 'tamarisk 'blood' (i.e. resin)': NW 5'

dām erēni (MÚD gišEREN) 'cedar 'blood' (i.e. resin)': IGI 2: 24

dām kurșipti alpi șalmi (MÚD kur-șib-ti $\mathrm{GU}_{4} \mathrm{GE}_{6}$ ) 'blood of a black ox-fly’: IGI 2: 214' damqu $\left(\mathrm{SIG}_{5}\right)$ 'good' $\rightarrow$ himèti damiqte

dāmātu (ŠIM.BI.SIG $7 . \mathrm{SIG}_{7}$ ) 'dāmātu-paste’: IGI 1: 42', 45'

dāmu (syllabic, MÚD or MUD) 'blood': IGI 1: 36', 40’, 45’, 48', 61', 68', 71', 74', 75’, 77', 79', 99', 178'; IGI 2: 25; NR 3', 5', 6', 10'; NW 4'; NZ 4'; NZa 8' $\rightarrow$ dām kurșipti alpi

șalmi; dāma ša libbi šahî; pursìt dāmi; 'blood' of a plant (i.e. resin)' $\rightarrow$ dām erēni/bīni

dāmu ša libbi šahî (MÚD ša ŠÀ ŠAH) 'blood from a pig’s 'heart': IGI 1: 48'

dannu (KALA.GA) 'strong': IGI 1: 81'

daqqiqu (syllabic) ‘tiny’ $\rightarrow$ zēr šammi daqqiqi

diga/ilu $\rightarrow$ diglu

digil īnī (di-gi-il IGI.MIN) 'eyesight': IGI 3: 51'

diglu (syllabic) 'vision': IGI 1: 80'; IGI 2: 124', 126' $\rightarrow$ digil ìnī

dimtu (ÉR) ‘tear(s)’: IGI 1: 79’, 137’; IGI 2: 58', 121'; NK 10’; NQ 5’, 10’; NS 1'; NV 6'; NZa 8' $\rightarrow$ naqû 
diqāru (dứ̛́TUL) ‘diqāru-pot’: IGI 2: 63’, 68’

dišip šadê/î (LÂL.KUR.RA, LÀL.KUR(-e/i)) ‘mountain honey’: IGI 1: 40’, 64'; IGI 2: 18, 62', 120', 154', 163'; IGI 3: 50', 53', 69'; NS 7'

dišpu (LÀL) 'honey': IGI 2: 20, 51', 148', 195'; IGI 3: 48', 67'; NF 16'; NS 4' $\rightarrow$ dišip šadê, dišpu peșû

dišpu peșû (LÀL BABBAR) 'white honey’: IGI 2: 195', 215’; IGI 3: 1

dudubi (DÙ.DÙ.BI) 'its ritual, medical application': IGI 1: 69', 72', 97’, 109', 117', 124', 131', 135', 143', 146', 148', 149', 154', 156', 158', 162', 180', 185'; IGI 3: 27', 84', 87'; NF 44'; NZb 3'; NY 7'

\section{E}

ebbu (syllabic and DADAG) 'pure': IGI 1: 103', 122'; NF 23'

edeppu (syllabic) 'to blow, inflate'

D-stem: ud-du-pu: IGI 1: 174'; ud-du-pa-a-ma: NK 9'

edû (syllabic) 'known': IGI 2: 14

edû (syllabic) 'to know'

G-stem: i-di/du-u: IGI 1: 152': ti-di: IGI 1: 152’

egemgīru (syllabic and 'išNÍG.GÁN.GÂN) 'egemgīru-plant': IGI 2: 113’ $\rightarrow$ zēr egemgīri

egû (syllabic) 'to become lazy/tired'

G-stem: e-gu-ma: IGI 1: 30'?

ēkallu (É.GAL) 'palace': IGI 2: 217', 222'

elēnu (UGU-nu, AN.TA) 'above': IGI 1: 127'; IGI 2: 64'

eli (syllabic and UGU) 'on, over, above': IGI 1: 31'; IGI 2: 72', 119'; IGI 3: 68' $\rightarrow$ muhhu ellu (KÙ) 'clean, holy': IGI 1: 160', 173'; NT 9' $\rightarrow$ libbi elleti gišimmari

elû (syllabic) 'go up, get out, ascend'

G-stem: li-la-a: IGI 1: 198'

Š-stem: šu-li-i: IGI 1: 193', 199'; tušellâmma(E $\left.{ }_{11}-m a\right)$ : IGI 2: 28, 59', 79', 103’; NR 8';

tu-še-li-ma: NF 27'

èma (syllabic) 'whenever': IGI 1: 69', 97', 109', 117', 144', 154', 186'; IGI 3: 89'; NU 15'

emèdu (syllabic) 'to lean on, apply'

D-stem: tu-mad: IGI 2: 185'

Št-stem: tuš-te-mid: IGI 1: 83’

emesallu ( ${ }^{\mathrm{mun}}$ eme/me $e_{5}(\mathrm{~A})$-sal(-lì), ${ }^{\mathrm{mun}}$ eme-sal-la, A.MEŠ-sal, me $e_{5}(\mathrm{~A})$-SILIM, ${ }^{\mathrm{mun}}$ eme-sala)

'emesallu-saline solution': IGI 1: 41', 61', 82', 86'; IGI 2: 8, 10, 23, 57', 116', 146', 195';

IGI 3: 50', 52', 58'; NK 8'; NQ 12'; NS 2', 7'; NW 3'

eтmu (syllabic) 'hot': IGI 2: 3

enqu (syllabic) 'expert': IGI 1: 75'

enūma (UD-ma) 'when': IGI 1: 17’; IGI 2: 71', 157’; IGI 3: 2; NF 13’; NT 12'

enzu (UD) ' 'goat': IGI 3: $10 \rightarrow$ šizbi enzi

eperu (SAHAR) 'dust': IGI 1: 138' 
epēšu (syllabic and DÙ(-šú/uš)) 'to do, make'

G-stem: tēpušu(DÙ-šú): IGI 1: 17’; teppuš(DÙ-uš): IGI 1: 63’; IGI 2: 24; teppušma(DÙma): IGI 3: 36', 45’; NT 3'; te-pu-uš: IGI 3: 53’; e-pu-uš: IGI 1: 152'

Gtn-stem: tēteneppuš(DÙ.DÙ-uš): IGI 1: 9' ; tēteneppuš(DÙ.MEŠ): IGI 1: 85' epû (syllabic) 'bake'

G-stem: te-ep-pi: IGI 2: 65’; te-pi: NR 8’

eqû (syllabic and MAR) 'to daub, instil'

G-stem: teqqi(MAR): IGI 1: 11', 14', 15', 20', 33', 34', 35', 38', 40', 41', 44', 45', 46', 47', 49', 58', 60', 63', 64'

IGI 2: 4, 18, 20, 21, 24, 50', 51', 60', 61', 64', 66', 70', 71', 72', 73', 74', 75', 81', 83', 84', 93', 95', 99', 101', 102', 103', 104', 116', 119', 123', 127', 129', 139', 141', 142', 143', 144', 146', 147', 148', 150', 151', 154', 155', 157', 158', 161', 162', 164', 167', 168', 170', 173', 178', 187', 192', 193', 194', 208'

IGI 3: 6, 16', 17', 19', 30', 50', 53', 54', 95', 103'; NF 7', 12'; NP 2'-5', 7', 8'; NR 2', 4', 5'; NS 4', 8'; NU 6', 9'

te-(eq)-qí: IGI 1: 2', 4', 17', 22', 25', 28', 39', 41', 69'

IGI 2: 53', 85'-88', 97', 107', 114', 180', 182'-184', 186'

IGI 3: 69'; NF 2', 3', 16'

Gtn-stem: tēteneqqi(MAR.MEŠ): IGI 1: 13', 55’; IGI 2: 7, 55', 99', 125’, 136'; IGI 3: 43’, 61', 65', 67’, 83’; te-te-né-eq-qí: IGI 1: 54'; te-te-né-qí: IGI 2: 82', te-te-neq-qí: IGI 2:

120 '

erbēšeret (14) 'fourteen': IGI 1: 69'

erbēt (4) 'four': NK 13', 14'

erēbu (KU $\left.\mathrm{K}_{4}-u b\right)$ 'enter, go down, set (for sun)'

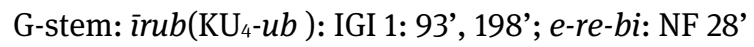

erēnu (EREN) 'cedar tree'; NF 34': $\rightarrow$ dām/šaman erēni

erēpu, arāpu (syllabic) 'to cloud over, grow dark'

G-stem: e/i-ru-pu: IGI 1: 30’; ar-pa: IGI 2: 61'

erēru 'to dry out'

G-stem: ir-ri-ra: IGI 2: 99'

erru I (syllabic) 'parched': IGI 2: 2

errû ('ÚKUŠ.LAGAB) 'errû-colocynth': IGI 2: 122'; IGI 3: 52' $\rightarrow$ inib errî

eršu ( 'išs NÁ) 'bed': NF 31'

$\bar{e} \boldsymbol{r u}$ ( ${ }^{\mathrm{giš} M A . N U) ~ ‘ e ̄ r u-t r e e ’: ~ N F ~ 18 ’ ~} \rightarrow$ arti/šuruš/zēer ēri

erû (URUDU) 'erû-copper’; IGI 3: 6; NF 12’ $\rightarrow$ erû labīru, hīl erî

erû ( $\left.\mathrm{TI}_{8}{ }^{\text {mušn }}\right)$ 'erû-eagle': $\rightarrow$ muhhu ša erî

erû labīru (URUDU SUMUN) 'old copper’: IGI 2: 99', erû zikaru (URUDU NITA) 'male copper (bead)’: IGI 3: 4

esēpu (syllabic) 'to collect'

G-stem: te-sip: IGI 2: 28, 174'

eședu (syllabic) 'to harvest'

G-stem: e-ṣi-id: IGI 1: 197’; ina e-șe-di-šú: IGI 1: 197’ 
eșemti amēlūti (GÌR.PAD.DU NAM.LÚ.U $19 . L U)$ 'human bone': NK 3'

eșemtu (GÌR.PAD.DU/DA) 'bone': IGI 1: 6'; NZ 22' $\rightarrow$ eșemti amēlūti, lipi eșemti arikti,

lipi eșemti, lipi eșemti kurīti immerim, lipi eșemti kurīti ša immeri, lipi eșemti

immeri, lipi eșemti șabīti, muhhu ša eșemti kurīti immeri

eșēpu (syllabic) 'to twine thread (two-ply)'

G-stem: te-eș-și-ip: IGI 1: 185'

ešeret (10) 'ten': IGI 1: 8', 12'; NF 35'

ețemmu (GIDIM) 'ghost': NT 5'; NU 12'; NZb 8' $\rightarrow$ harbātu

ețlu (GURUŠ) 'young man, lad': IGI 1: 159', 161', 198'

ețû (syllabic) 'to become dark'

Gtn-stem: i-te-né-ța-a: IGI 2: 54'

ezzu (syllabic) 'anger': NZa 9'

\section{G}

gabīdu (syllabic) 'liver’: $\rightarrow$ makūt gabìdi (ša imēri)

gabû (syllabic and IM.SAHAR.NA4.KUR.RA) 'gabû-alum': IGI 2: 161' $\rightarrow \boldsymbol{a} \boldsymbol{b a n}$ gab̂

galābu (syllabic and SAR(-ab)) 'to shave'

D-stem: tugallab(SAR-ab): IGI 1: 24'; NZ 21'; tu-gal-lab: IGI 2: 7, 109', 118'

giddagiddû (GÍD.DA.GÍD.DA, GÍD.DA.GÍD- $d u$, GÍD.GÍD) 'giddagiddû-fibers': IGI 2: 12, 13,

15

gišimmaru ( ${ }^{\text {iš }}$ GIŠIMMAR) 'date palm': NF 18', 21' $\rightarrow$ libbi elleti gišimmari, šuruš

gišimmari zikari, taltallû ša gišimmari

gišrinnu (GIŠ.ÉRIN) 'scale'; NS 4'

gulgullu (syllabic) 'skull' $\rightarrow$ kalli gulgul amēli

gūqānu $\rightarrow q \bar{u} q \bar{a} n u$

gūru ša qanî (gu-ra šâ GI) 'reed leaf': IGI 2: 109'

gurušgaraš ša šahî (gu-ru-uš-ga-ra-aš ša šahî) 'vulva of a pig': IGI 2: 27

H

habburru (syllabic and HÉNBUR) 'sprout': IGI 1: 195', 196' $\rightarrow$ habbur še'i

habbur še'i (HÉNBUR ŠE.AM) 'grain-sprout': IGI 1: 10'

hallu (syllabic) 'hind leg' $\rightarrow$ larsīni ša halli lahri ša šumēli ša māšī ūlidu

hallūru (GÚ.GAL) 'chickpea' $\rightarrow$ qēm hallūri

hamātu (syllabic) 'to burn, inflame'

G-stem: ha-an-ța: IGI 1: 14'; IGI 2: 9; hamiț(TAB.BA): IGI 3: 49'; NS 6'

hamiš $\rightarrow$ hamšat

hamiššer (15) 'fifteeen': IGI 2: 139', 141'

hamšat, hamiš (5) 'five': IGI 1: 29', 44'; IGI 2: 92' 
hāmū (syllabic) 'chaff': IGI 1: 187', 193'

handabillu (syllabic) 'handabillu-shell': IGI 1: 188'

hapāpu (syllabic) 'to rinse'

D-stem: tu-ha-pap: IGI 3: 31'

harāṣu (syllabic) 'to break off, cut into'

G-stem: ta-har-ra-aṣ: IGI 3: 81', 90'?

harbātu (syllabic) 'abandoned land'; NT 5' $\rightarrow$ ețemmu

harištu (syllabic) 'woman in labour': IGI 1: 104'

hașab igulê (ŠIKA i-gu-le-e) 'potsherd of fine salve': IGI 2: 134'

hașab nurmî (ŠIKA gišNU.ÚR.MA) 'pomegranate peel': IGI 2: 116'

hașab țābti (ŠIKA MUN) 'flake of salt': IGI 2: 194'

haṣbu labīru ša kibša išû (ŠIKA LIBIR.RA šá ki-ib-ša? TUK-ú) 'old fungus-ridden

potsherd': IGI 2: 62'

hașbi šammi peșî (ŠIKA Ú.BABBAR) 'peel of white plant': IGI 2: 139'

hașbu (ŠIKA) 'potsherd, sherd, peel, flake': IGI 2: 141'; NZ 17' $\rightarrow$ hașab

nurmî/țābti/igulê, hașbu labīru ša kibša išû, hașbi šammi peșî

hašālu (GAZ) 'to crush'

G-stem: tahaššal(GAZ): IGI 1: 16’; IGI 2: 56', 153', 162', 168'; IGI 3: 5, 72'; NF 11'

hašhūru (iišHAŠHUR) 'apple': IGI 1: 47'; NF 18', 21'

hašlu (syllabic) 'crushed': IGI 1: 170'

hašû (syllabic) 'to chop (foodstuffs)'

D-stem: ú-haš-šá: IGI 1: 26'

hašû ('HAR.HAR) 'hašû-thyme': IGI 3: 100'; NU 4', 10'

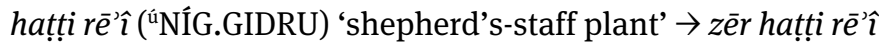

hepû (syllabic) 'to break'

G-stem: te-he-pi: IGI 1: 160'

herșu (syllabic) 'cut (in the liver)': IGI 3: 89'

hesû (syllabic) 'to squeeze out drugs'

D-stem: tu-ha-sa: IGI 1: 80’; IGI 2: 63’

hīl abukkati (ILLU LI.TAR/DUR) 'gummy resin of abukkatu-plant': IGI 1: 78'; IGI 2:

208'; IGI 3: 13, 27'

hīl baluhhi (ILLU šmBULUH) 'resin of baluhhu-aromatic': IGI 2: 138'

hīl erî (ILLU URUDU) 'exudation of copper': IGI 1: 13', 82', 87'

hīl nuhurti (ILLU NU.LUH) 'resin of nuhurtu-plant': IGI 2: 137'

hìl tīyati (ILLU 'ti-iá-tu) 'resin of tīyatu-plant': IGI 3: 52'

hīlu (ILLU) 'resin' $\rightarrow$ hīl abukatti/baluhhi/erî/nuhurti/tīyati

himēti damiqte (İ.NUN SIG - -te) 'good quality ghee': IGI 1: 16'

himētu (İ.NUN(.NA)) 'ghee': IGI 1: 11', 13', 14', 25', 28', 33', 34', 35', 38', 41', 42', 44', 45', 46', 58', 60', 72', 84'; IGI 2: 18, 21, 51', 55', 58', 66', 70', 72', 76', 79', 81', 99', 119', 120', 139', 141', 142', 143', 144', 148', 150', 152', 157', 163', 165', 168', 198', 215'; IGI 3: 1, 38', 48', 50', 67', 69', 83', 103'; NF 16'; NR 4', 8'; NS 8'; NW 5' $\rightarrow$ himēti damiqte hulālu ( $\left.{ }^{\mathrm{na} 4} \mathrm{NÍR}\right)$ 'banded agate (onyx)': IGI 1: 103’, 122' 
huliam (syllabic) 'huliam-vessel': IGI 1: 81', 83'

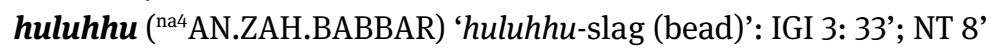

hurāṣu (KUG.GI) 'gold'; NF 20' $\rightarrow$ aban hurāṣi

hūratu (gišHAB) 'dyeing sumac': IGI 2: 4

hușābu (syllabic) ‘twig’: IGI 1: 189’, 193’

¡, ya (syllabic) 'my’: IGI 1: 66', 74'; IGI 2: 219', 220'

idru (syllabic) 'idru-saltpetre': NZ 13'

igāru (É.GAR8) 'wall' $\rightarrow$ șurār igāri

igulû (syllabic) 'fine ointment': $\rightarrow$ hașab igulê

ikil pāni (syllabic) ‘darkness of face, gloom': IGI 1: 130’

iklu (syllabic) 'gloominess, darkness' $\rightarrow$ ikil pāni

iltānu (IM.2) 'north wind': IGI 1: 115'

imbu' tâmti (KA.A.AB.BA) 'algae': NF 19', 22'; NU 3'

imēru (ANŠE) 'donkey': $\rightarrow$ makūt gabìdi ša imēri

imhur-ešrāa ('IGI-NIŠ) 'imhur-ešrā-plant': NU 4'

imhur-līm ('IGI-lim) 'imhur-lìm-plant': IGI 2: 142'; NF 45'; NU 4'

imittu (ZAG, 15) 'right side': IGI 1: 146'; NV 6' $\rightarrow$ in imittǐšu; ana imitti

immeru (UDU, UDU.NÍTA) 'sheep, male sheep': IGI 1: 91'; IGI 2: $18 \rightarrow$ birki immeri,

kabūt immeri, lipi eșemti immeri, lipi eșemti kurî immeri, lipi eșemti kurî ša

immeri, lipi kalìti immeri, marat immeri, muhhu ša eșemti kurî immeri, sikkat șēli immeri

in imittǐšu (IGI(.MIN) ZAG/15-šú) 'man's (lit. his) right eye': IGI 2: 200'; NV 6', NZa 8' in šumēlišu (IGI GÙB-šú) 'man’s (lit. his) left eye’: IGI 2: 202'

ina (AŠ and syllabic) 'in, on': IGI 1: 7', 10'-16', 18'-20', 22'-29', 33'-35', 37', 38', 41'47', 52', 57', 58', 60', 62'-65', 67', 69', 72', 78', 79', 81', 84', 86'-88', 97', 109', 118', 144', 146', 148', 154', 161', 163', 168', 181', 186', 194', 200'

IGI 2: 1, 2, 4, 7, 16, 17, 18, 20, 21, 24, 28, 50', 51', 53', 54', 55', 56', 58', 59', 61'-66', 68', 69', 70'-78', 80'-82', 85', 86', 88', 93', 94', 97'-99', 101'-104', 111', 113', 116'120', 123', 125', 139'-142', 144', 145', 148'?, 150', 152'-155', 157'-159', 162', 163', 165', 167', 168', 174', 180', 181', 184', 185', 192'-195', 198', 205', 215', 221'

IGI 3: 1, 6, 28', 29', 31', 35’, 36’, 38', 39', 40’, 44', 48’, 50’, 53’, 56’, 67’, 69', 71', 72’, 75', 76', 88', 100', 103'

NF 5', 11', 15', 16', 19', 20', 22', 27', 28', 33', 35', 44', 46'; NK 4', 7', 12'; NQ 8'; NR 3'5', 7', 8'; NS 4', 7'; NT 3', 4', 6; NU 2'-4', 10', 13', 15'; NV 2', 4’; NW 5', 6’; NZb 11'; NZ 2', 10', 11', 18', 20'

'through, with': IGI 1: 7’, 38', 84', 86'-88', 160', 173'; IGI 2: 2, 7, 73', 105', 115', 117', 132', 166', 201', 203'; 'over (charcoal)': IGI 1: 33'; IGI 2: 74', IGI 3: 64', 100', 103'; NK 5’, NU 8'; NY 4'; 'from': IGI 1: 67’, 174', 180', 188', 189'; IGI 2: 17; NU 9'; NW 4'; 
'under': IGI 1: 81'; IGI 2: 28, 59', 103’; IGI 3: 34'; NF 19', 22'; NU 7'; 'among': IGI 2: 219'; 'during, when': IGI 3: 28'; NZa 10'

$\rightarrow$ ina arkīti, ina beri/u-, ina libbi, ina pāni

innā/i (IGI.MIN) '(both) human eyes': IGI 1: 4', 7', 10'-15', 17', 18', 20', 21', 22'-23', 25', 26', 28', 29', 32'-46', 48'-50', 56', 57', 59', 61', 63', 64', 68', 71', 72', 77', 78', 79', 80', 84'-88', 90', 91', 99', 105', 109', 111', 112', 123', 136'-138', 165'-167', 172', 184', 187', 193', 199', 200', 201'

IGI 2: 1-4, 7, 9-12, 16-19, 22, 24, 25, 27, 52', 53'-58', 60', 61', 63', 66', 69', 76', 78', 89', 91', 97'-99', 101', 105' (raven's eyes), 108', 115'-117', 119', 121', 123'-127', 132', 136', 137', 139', 142', 145', 148', 158', 159', 166', 169', 175', 181', 185', 190', 191' 194', 196', 199', 204', 207', 208', 213', 215', 216'

IGI 3: 1, 2, 6, 8, 9, 26', 30’-32', 35’, 37’, 39', 43’, 49’-51', 53’, 54', 57’, 61', 67’, 68', 70', 75', 78', 81', 83', 98', 103'

NF 7', 12', 16'; NK 6'-9', 15'; NP 7'; NQ 5', 7', 8', 10'; NR 2', 3', 5', 6', 10'; NS 1', 4', 6', 8'; NT 4'; NU 5', 6'; NV 1'; NZb 6'; NZ 4', 8'; NZa 8';

'vision': IGI 2: 17

$\rightarrow$ digil ìnī, lamassat ìnī, libbi īnīšu, rēš ìnī ina arkīti (ina EGIR-tì) 'afterwards': IGI 2: 4' ina beri/u- (syllabic) 'in between': IGI 1: 93', 101', 120', 126', ina libbi (ina ŠÀ) 'from the middle of': IGI 1: 79', 94'; 'into': IGI 2: 147’; NU 5', 9' ina muhhi (ina, i-na UGU) 'over': IGI 3: 6; NF 12' ina pāni (ina IGI) 'in front of': IGI 3: 9, NS 4' inbu (GURUN) 'fruit' $\rightarrow$ inib errî inib errî (GURUN úÚKUŠ.LAGAB) 'fruit of errû-colocynth': IGI 1: 22', IGI 2: 162' inu (syllabic and IGI) f. 'eye’: IGI 1: 96', 108', 116', 118', 124', 126' (eyes), 131', 135' (136'138'), 145', 149', 153', 156', 158', 159', 161', 162', 163', 173', 174'-176', 179', 181', 198';

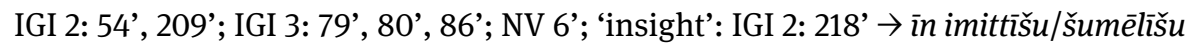
irri abāri (ir-ri A.GAR 5 ) 'lead salve': IGI 2: 4, 97' isqūqu (Zİ.KUM) 'coarse flour': IGI 1: 57'; NZ 22' ișșūr hurri (MUŠEN.HABRUD) 'partridge': IGI 3: 42' išātu (IZI) 'fire': NF 15'; NT 6'

iškūru (GAB.LÀL) 'wax': IGI 1: 33'; IGI 2: 53', 58'; IGI 3: 42', 97’, 103’ iškūru peșû (GAB.LÀL BABBAR) 'white wax': IGI 3: 100'

ištēn (1) 'one, first': IGI 1: 41', 201'; IGI 2: 54', 140', 141'; IGI 3: 49'; NS 6'; NT 8'-11' ištēniš (1-niš and UR.BI) 'together': IGI 1: 78', 83', 154'; IGI 2: 23, 53', 87’, 113', 123', 215’;

IGI 3: 1, 5, 12, 48', 72', 83', 95’; NF 11'; NK 12'; NR 7'; NU 9'; NZ 18', 24 ' ištēššeret (11) 'eleven': IGI 2: 123'; IGI 3: 34', 43’ ištiššu (1-šú) 'once' $\rightarrow$ ištiššu šinišu šalā̌̌išu ištiššu šinišu šalāšišš (1-šú 2-šú 3-šú) 'once, twice, three times’: IGI 1: 15’; IGI 2: 3, 143' ištu (syllabic) 'from': IGI 1: 164'; IGI 2: 220' išû (TUK) 'to have': iš̂̂(TUK-ú): IGI 2: 62'

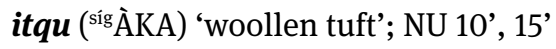


itqūr abāri (DÍLIM A.BÁR) 'lead spoon(salve)': IGI 1: 20’, 25’; IGI 2: 93’, 119’, 167’; IGI 3:

30', 54', 93', 105'

itqurtu (syllabic) 'a spoon': IGI 3: 109'

itti (syllabic, and KI) 'with': IGI 1: 178'; IGI 2: 52', 91'; IGI 3: 72'; NT 1'

ittu (syllabic) 'an ominous sign': IGI 1: 67'

ittû (syllabic) 'seeder-plough': IGI 1: 195'

ițțû (ESIR) 'liquid bitumen’: IGI 3: 94’

itūlu (NÁ-al) 'to lie down' $\rightarrow$ nâlu

Š-stem: 'to pickle, cure': tuštāl(NÂ-al): IGI 2: 71'; NR 3'

izuzzu (syllabic and GUB) 'to stand, be present'

G-stem: i-ši-za-nim-ma: IGI 1: 198'; izzazzu/ū (GUB-zu): NP 1’; izzizzu (GUB-zu): NF

15'; NT 6'; izziz(GUB-iz): NR 3', 5', 10'

Š-stem: tuš-za-as-su: IGI 3: 78'

K

$k a$ 'you, your': IGI 2: 73', 80'; NZb 9', 10' $\rightarrow$ ki

$k a b a \overline{b u}$ (syllabic) 'to burn'

D-stem: tu-kab-ba-ab: NF 15'; NT 6'

kabātu (DUGUD) 'to become heavy'

G-stem: ikabbitūšu(DUGUD-šú) IGI 1: 80'; kabit(DUGUD): IGI 2: 124', 126', 137’;

ikabbitā(DUGUD): IGI 3: 68'

kabūt alpi (ŠURUN GU 4 ) 'ox dung': IGI 3: 72' $\rightarrow$ kabūt Šeriš

kabūt Šeriš (ŠURUN d ${ }^{\mathrm{G} U} \mathrm{U}_{4}$ ) 'ox dung': NU 10' $\rightarrow$ kabūt alpi

kabūt immeri (ŠURUN UDU) 'sheep dung': IGI 1: 37'

kabūtu (ŠURUN) 'dung' $\rightarrow$ kabūt alpi/immeri/Šeriš

kadādu (syllabic) 'to rub in'

G-stem: ta-kad-da-ad: IGI 2: 2

kakkabu (UL and MUL) 'a star': IGI 1: 81', 189'; IGI 3: 34'; NF 19', 22'; NU 7'

kakkû (GÚ.TUR) 'lentil’ $\rightarrow$ qēm kakkî

kala $\rightarrow$ kalû

kala ūme 'every day': IGI 2: 2, 5; IGI 3: 60'

kalbānu (gišsMAŠ.HUŠ) 'kalbānu-plant (lit. dog-like plant)': IGI 2: 5

kalbu (UR.GI ${ }_{7}$ ) 'dog' $\rightarrow$ lišān kalbi

kalgukku ('imAL.GUG) 'kalgukku-red paste': IGI 2: 84'; NF 19'; NU 2'

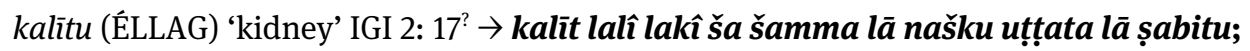

lipi kalīti alpi; lipi kalīt alpi șalmi; lipi kalīti immeri; lipi kalīti sāmu

kalìt lalî lakî ša šamma lā našku uț̣ata lā șabitu (ÉLLAG MÁŠ.TUR la-ki-i ša Ú la na-

$a s ̌-k u$ ŠE.BAR la șa-bi-tú) 'kidney of young suckling goat which has not (yet) bitten

a plant nor grasped a grain kernel': IGI 2: 111'

kalli gulgul amēli (kal-li gul-gul LÚ.U $\left.{ }_{19} . \mathrm{LU}\right)$ 'cranium of a human skull': NK 4' 
kalû ('imKL) 'kalû-paste': NF 19', 22'

kalû (syllabic) 'to hold (back)'

G-stem: ak-lá: IGI 1: 67’

kalû (syllabic and DÙ.A.BI) 'everything, the whole of': IGI 3: 73', 74' $\rightarrow$ kala ùme

kamkadu (syllabic) 'kamkadu-plant': IGI 2: 108'

kammu (syllabic) 'kammu-tanning-fungus: IGI 2: 17; IGI 3: 6, 100’, 105’; NF 12'; NR 7’ $\rightarrow$

kammu ša aškāpi

kammu ša aškāpi (kam-mu šá AŠGAB) ‘kammu-tanning-fungus of a leather worker’:

IGI 1: 33’; IGI 3: 102’

kamūnu ('GAMUN) 'cumin' NK 2'

kanaktu ( $\left.{ }^{\mathrm{Sim}} \mathrm{GIG}\right)$ 'kanaktu-aromatic': IGI 2: $23 \rightarrow$ lipi kanakti

kanānu $\rightarrow$ qanānu

kannu ( $\left.{ }^{\mathrm{dug}} \mathrm{GAN}\right)$ 'kannu-jar': IGI 1: 122'

kannu (syllabic) 'seedling': IGI 1: 196'

kânu (GIN and syllabic) 'to establish, deposit, to set up'

D-stem: ú-kin: IGI 2: 222'; tukān (GIN-an): IGI 3: 76'; NF 17'

kapātu (syllabic) 'to roll together (into a pill)'

D-stem: tu-kap-pat: IGI 1: 27'

kappu (syllabic and PA) 'eyelid': IGI 2: 97', 98', 108'

karān šēlebi ('GEŠTIN.KA.$A$ ) 'fox-vine': IGI 2: 63', 73', 86', 100', 112', 120'

karānu (GEŠTIN.NA) 'wine': IGI 1: 57'

karpat țābāti (DUG A.GEŠTIN.NA) 'vinegar vessel': IGI 1: 92'

karpatu (DUG) 'a vessel': IGI 1: 103', 122'

kâru (syllabic) 'to rub'

G-stem: ta-kar-ma: IGI 1: 85'

kaspu (KÙ.BABBAR) 'silver': NF 20'

kasû (syllabic and GAZI ${ }^{\text {sar }) ~ ' k a s u ̂-p l a n t ’: ~ I G I ~ 1: ~ 10 ’, ~ 32 ’, ~ 37 ’, ~ 170 ’ ; ~ I G I ~ 2: ~ 9, ~ 11, ~ 66 ', ~ 69 ’, ~}$

119'; IGI 3: 41', 94', 97', 99', 102', 107'; NK 16'; NR 11' $\rightarrow$ mêe/qèm kasî

kașāru (syllabic and KEŠDA) 'to tie, knot'

G-stem: takașșar(u)(KEŠDA): IGI 1: 69', 97', 109', 117', 144', 154', 186'; kàṣ-rat: IGI 1:

127

D-stem: tu-ka-șar: IGI 1: 161'

kâșu (syllabic) 'to skin, grate' $\rightarrow$ kịṣu

G-stem: ta-ka-aș: IGI 2: 65'

kașû (SED) 'cold': IGI 2: 114'

kașû (syllabic) 'to be cold'

D-stem: tu-kàṣ-ṣa: IGI 1: 24'; IGI 2: 94', 118'; li-ke-eṣ-șa-a: IGI 1: 105’

kašādu (syllabic) 'to reach'

G-stem: ik-šu-du-ki-na-ši: IGI 1: 190’

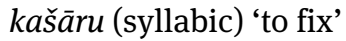

G-stem: kuš-ri-in(-ni): IGI 1: 74'

kašû (syllabic) 'to increase, benefit' 
G-stem: ik-ši: IGI 2: 97’

katāmu (syllabic and DUL) 'to cover objects, close the eye'

G-stem: katmā(DUL-(ma)): IGI 1: 21', 37’, 85’; IGI 2: 76'; takattamma(DUL-ma): IGI

2: 64'; kát-ma: IGI 2: 159'

kayyamānamma (syllabic) 'regularly, again and again': IGI 3: 6; NF 12' $\rightarrow$ kayyamān,

kayyānam

kayyamānu (SAG.UŠ) 'regularly, again and again': IGI 2: 75', 78' $\rightarrow$ kayyamānamma,

kayyānam

kayyānam (syllabic) 'regularly, again and again': IGI 2: 82' $\rightarrow$ kayyamānamma, kayyamānu

ka'inimma (KA.INIM.MA) 'invocation': IGI 1: 68', 71', 77', 96', 108', 116', 124', 131', 135', 149', 153’, 156', 158', 162', 175', 179', 184', 193’, 199’; IGI 3: 26’; NZb 2'

$k i$ 'your' (f.): IGI 1: 95', 107', 160' $\rightarrow k a$

kiam (syllabic) 'so, thus': IGI 3: 82'; NF 13'

kibrītu (PIŠ ${ }_{10}{ }^{-}$'ID) 'kibrītu-sulphur': IGI 3: 94', 105', 106'; NU 14'

kibšu (syllabic) 'fungus': IGI 2: 62'

kibtu (GIG) 'wheat' $\rightarrow$ qèm kibti

kidkidbi (KÌD.KÌD.BI) 'its ritual': IGI 1: 78'

kikkirânu (šimšE.LI) 'kikkirânu-aromatic': IGI 1: 21'

kilaltān (syllabic) 'both’: IGI 1: 39'

kìma (syllabic and $\mathrm{GIN}_{7}$ ) 'like, as’: IGI 1: 91', 92', 189', 190'; IGI 2: 12, 14, 67’, 99'; IGI 3:

6, 8, 9, 10, 37', 93'; NF 12', 28'

kināši (syllabic) ‘to you': IGI 1: 113'-115', 190'

kirbānu (syllabic) 'lump, clod': IGI 1: 188'

kìru (syllabic) 'oven': IGI 1: 65'

kišādu (GÚ) 'neck': IGI 3: 35', 76'; NU 4', 10', 16'

kișirtu (syllabic) 'thickening, brow': IGI 1: 127'

kișru (syllabic and KA.KEŠDA) 'knot': IGI 1: 69', 97', 109', 117', 144', 154', 186'; 'a bud':

IGI 1: 196'

kișșatu (syllabic) 'skin condition': NV 1' $\rightarrow$ kịsu

kịsu (syllabic) 'grated': (sahlê kiṣ̂ati) IGI 2: 98'; 'flayed, skinned, description of the

irritated area around the eyes': (inni kiṣ̂ati) IGI 2: 98' $\rightarrow$ kâṣu, kișșatu

kiššatu (ŠÚ) 'world': IGI 2: 217'

kukru (šm/giš GÚR.GÚR) 'kukru-aromatic': IGI 1: 62'; IGI 2: 11, 16; IGI 3: 11, 41', 93’, 97’,

100', 104', 110'; NK 16'; NQ 12'; NS 2'; NU 3'; NZ 14', 16' $\rightarrow$ qēm kukri

kullu (syllabic and DAB) 'to hold, contain, keep, retain'

D-stem: ú-kal: IGI 1: 85'; IGI 3: 60'; ú-kal-ma: IGI 2: 5; ú-kal-la: IGI 2: 12, 58'; NZ 4';

NZa 8'

kunšu (syllabic) 'wool braid': IGI 1: 8'

kunu (syllabic) 'your' (2 m. pl. gen. suff.): IGI 2: 13, 15

kupru (syllabic) 'kupru-dry bitumen': IGI 3: 106'

kurāru (GIG.GIR) 'kurāru-pustle': IGI 2: 108' 
kurkānû (syllabic and 'KUR.GI.RIN.NA) ‘kurkānû-plant’: IGI 2: 113’; NZb 5’

kurkû (KUR.GI ${ }^{\text {mušn }) ~ ' g o o s e ': ~} \rightarrow$ šaman kurkî

kursinnu (syllabic) 'ankle, knuckle' $\rightarrow$ lipi kursinni șabīti

kurșipti eqli (kur-șip-ti A.ŠÀ) 'field butterfly’: IGI 2: 159'

kurșiptu (syllabic) 'butterfly’ $\rightarrow$ dām kurșipti alpi șalmi, kurșipti eqli

kurû (LÚGUD.DA) 'short': - lipî eșemti kurīti, lipî eșemti kurīti immeri, lipi eșemti

kurīti ša immeri, muhhu ša eșemti kurīti immeri

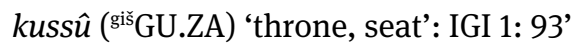

kūṣu (EN.TE.NA) 'winter': IGI 1: 63'; NK 14'; NR 7'

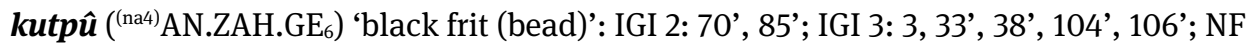
9’; NT 8'

lā (syllabic and NU) 'no, not, without': IGI 1: 23', 36', 70', 94', 104', 128', 129', 145', 191', 200’; IGI 2: 1, 6, 14', 65', 99’, 111’, 117', 219’; IGI 3: 49’, 66’, 68', 70’, 73’, 74’; NF 17’, 20', 23'; NS 6'; NW 4'; NZ 3'; NZa 10' $\rightarrow$ lām, lā (m) patān

labāku (syllabic) 'to be(come) soft'

D-stem: tu-la-bak 'you soften up': IGI 2: 81', labânu (syllabic) 'neck tendons': IGI 3: 75'

labāru (SUMUN) 'to be(come) old, long-lasting'

G-stem: ilabbirma(SUMUN-ma): IGI 2: 54’

labātu $\rightarrow$ lapātu

labīru (LIBIR.RA) 'old': $\rightarrow$ erû labīru, haṣbu labīru ša kibša išû, šaman ișșūr hurri?

labīi

labtu (ŠE.SA.A) 'roasted grain': IGI 2: 6, 52'; IGI 3: 92' $\rightarrow$ qēm labti, sīkti labti

lahābu (syllabic) 'grows'

G-stem: i-li-hi-ib: NF 33'

lahru $\left(\mathrm{U}_{8}\right)$ 'ewe': $\rightarrow$ larsīni ša halli lahri ša šumēli ša māšì ūlidu

lakû (syllabic) 'suckling’: IGI 2: 111’ $\rightarrow$ kalìt lalî lakî ša šamma lā našku utțata lā

sabitu

lalû (MÁŠ.TUR) 'a kid, young goat': IGI 2: 111’ $\rightarrow$ kalīt lalî lakî ša šamma lā našku

uț̣ata lā șabitu

$l \bar{a}(m)$ patān (syllabic) 'before eating/meal': IGI 1: 27', 43'; IGI 2: 145'

lām (syllabic) 'before': IGI 1: 115', 190', 194'; IGI 3: 29' $\rightarrow$ lā, lā $(m)$ patān

lamassat īnī ('LAMA(.MEŠ) IGI.MIN) 'pupil of the eye': IGI 1: 80'; IGI 2: 121', 124', 207'

lamû (NIGIN) 'wrap up'

G-stem: talammi(NIGIN-(mi)): IGI 1: 39’; NU 10’

lapāpu (syllabic) 'to wrap, make a wad

G-stem: tál-pap: IGI 1: 180’; NU 15’; NZb 5’; ta-la-pap: NU 15’

lapātu (syllabic) 'to touch' 
D-stem: ina la-ba-ti-šá: IGI 1: 173'

larsīni ša halli lahri ša šumēli ša māšš ūlidu (la-ar-sì-ni ša hal-li U $\mathrm{U}_{8}$ ša GÙB ša

MAŠ.TAB.BA Ù.TU) 'a hoof of the left hind leg of an ewe which bore twin(s)': IGI 2:

110 '

larsīnu (syllabic) 'a hoof' $\rightarrow$ larsīni ša halli lahri ša šumēli ša māšĩ ūlidu

lâšu (syllabic and SILA $\left.\operatorname{Si}_{11}(-a s ̌)\right)$ 'to knead'

G-stem: talâš(SILA 11 -aš) IGI 1: 10', 15’, 19', 27’, 29', 37’, 84'; IGI 3: 56', 72'; NR 8'; NZ

5'-7', 10'; ta-la/là-aš: IGI 2: 114', 145', 181'

latāku (syllabic) 'to test (a remedy)'

G-stem: la-te-ek: IGI 3: 96’

lazāzu (syllabic) 'to endure, persist'

G-stem: la-zi-iz: NF 33'

lemnu (HUL) 'evil': NU 12'

lemuttu (HUL-tì) 'evil' $\rightarrow$ mukīl rēš lemutti

leqû (syllabic and TI(-i/qé), ŠU.TI) 'to take, accept, + ventive to bring'

G-stem: teleqqe(TI(-qé)): IGI 1: 83’, 185’; IGI 2: 101'; IGI 3: 87’; NF 15’; NT 6’; NU 13’;

NW 5'; NZ 1'; liqi(TI-(i)): IGI 1: 95', 107'; i-le-qu-ni-ku: IGI 1: 160'; le-qé-ma: IGI 1:

170’; il-qa-a: IGI 2: 106'; leqâa(TI.MEŠ): NK 6'

lēru (SAHAR.KÙ.GI) 'lèru-paste’: IGI 1: 40'

lètu (syllabic) 'cheek, side' $\rightarrow$ ana lèt

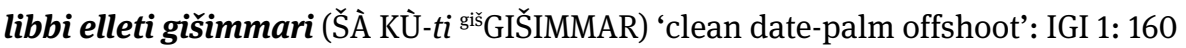
libbi īnišu (ŠÀ IGI.MIN-šú) 'inner part of patient's eyes': IGI 1: (26'), 36’, (42', 50', 56',

72', 84', 86'-88'); IGI 2: (2, 76', 78', 89', 115', 145', 158'), 159', (169'), 190', (194'); NU

5 ,

libbu (ŠÀ) 'middle, midst of'; 'internal organ/heart'; $\rightarrow$ a/ina libbi, dāmu ša libbi šahî,

libbi īnišu, ša libbi

libbu (ŠÀ) 'offshoot (of date palm)' $\rightarrow$ gišimmaru, libbi elleti gišimmari

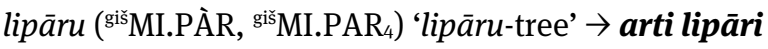

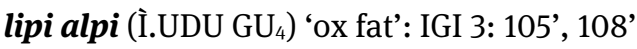

lipi eșemti (İ.UDU GİR.PAD.DU) 'bone marrow’: IGI 1: 108'

lipi eșemti arikti (İ.UDU GİR.PAD.DU GÍD.DA) 'marrow of a long bone': IGI 2: 53'

lipi eșemti immeri (İ.UDU GÌR.PAD.DU UDU) 'marrow of a sheep's bone': IGI 2: 70'

lipi eșemti kurīti (İ.UDU GÌR.PAD.DU LÚGUD.DA) 'marrow of a short bone': IGI 1: 43’

lipi eșemti kurīti immeri (İ.UDU GÌR.PAD.DU LÚGUD.DA UDU) 'marrow of a sheep's

short bone': IGI 2: 50

lipi eșemti kurīti ša immeri (İ.UDU GÌR.PAD.DA LÚGUD.DA ša UDU) 'marrow of a

short bone of a sheep': IGI 1: 6'

lipi eșemti șabīti (İ.UDU GİR.PAD.DU MAŠ.DÀ) 'gazelle bone marrow': NU 9'

lipi kaliti alpi (İ.UDU ÉLLAG GU 4 ) 'ox kidney fat': IGI 1: 95'; NK 4'

lipi kalit alpi șalmi (İ.UDU ÉLLAG GU $\mathrm{GU}_{6}$ ) 'kidney fat of a black ox': IGI 3: 6; NF 11' lipi kalīti immeri (İ.UDU ÉLLAG UDU.NÍTA) ‘sheep’s kidney fat’: IGI 1: 33’; IGI 2: 53’ lipi kalīti sāmu (İ.UDU ÉLLAG SA ) 'reddish kidney fat': IGI 1: 20' 
lipi kanakti (İ.UDU šm GIG) 'kanaktu-aromatic fat': IGI 1: 32', 62'; IGI 2: 25, 66’; IGI 3: 97' lipi kursinni șabīti (İ.UDU kur-sin-ni MAŠ.DÀ) 'fat from a gazelle’s knuckle’: IGI 2: 3 lipi nēši (İ.UDU UR.MAH) 'fat from a lion': IGI 3: 52'

lipi qanî (İ.UDU GI.MES) 'pith of reeds': IGI 3: 41'

lipi sāqi șabīti (İ.UDU sa-a-qí MAŠ.DÀ) 'fat of a gazelle’s thigh’: IGI 2: 147’

lipi șallamti (İ.UDU MUŠ.GE ) 'fat from a black snake’: IGI 3: 51’, 69'

lipištu (syllabic) 'fleshy substance’: IGI 2: 16

lippu (syllabic) 'wad, tuft: IGI 1: 180'; NU 15'

lipû (İ.UDU) 'fat': IGI 1: 11', 82'; IGI 2: 17', 22, 96', 158', 194'; IGI 3: 103’; 'bone marrow; pith of plants, aromatic fat' $\rightarrow$ lipi ...

lipû sāmu (İ.UDU SA s 'red fat': NR 3'

liqtu (syllabic) '(textual) material': IGI 2: 220'

lišāan kalbi ('EME.UR.GI ${ }_{7}$ ) 'dog's-tongue plant': IGI 1: 57' $\rightarrow$ mêe/zēr lišān kalbi

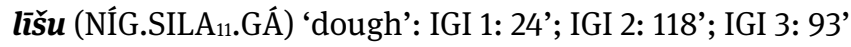

lu (syllabic) 'or’: IGI 2: 24

$l \bar{u}$... lū (syllabic) 'either ... or': IGI 3: 9, 10; NF 27’; NU 11', 12'

lubku $\rightarrow$ napšalti lubki

lulû (KÙ.GAN) 'lulû-antimony’: IGI 1: 6'; IGI 2: 70'; IGI 3: 33’

lumnu (HUL) 'evil’: NF 17’, 20’, 23’

\section{M}

$m a^{\prime} d a \rightarrow m a \bar{d} u$

ma (enclitic particle) 'and (then, thus)': IGI 1: 13', 23', 26’', 36', 38', 39', 79', 84', 85', 95', 105', 107', 118', 123', 151', 154', 163', 169', 181', 186', 192', 198'; IGI 2: 5, 6, 7, 12, 14, 28', 54', 59', 64', 79', 97', 99', 103', 117', 121', 123'-126', 131', 136', 137', 221'; IGI 3: 6, 9, 35', 36', 43’, 45’, 54', 57', 61', 65’, 67', 68', 70', 72', 82', 85', 87', 88', 90', 95’; NF 12', 14', 16', 20', 21', 23', 27', 33', 35'; NK 6', 9'; NR 3', 5', 8', 10'; NS 5’; NT 3'; NU 5', 6', 8', 12'; NV 3', 6'; NZb 1', 7'; NY 4'; NY 7a'; NZ 10'; NZa 8', 9'

enclitic particle marking nominal sentence: IGI 1: 14', 21', 25', 26'?, 30'?, 101', 126', 128', 129'?; IGI 2: 22; affirmative: IGI 1: 170-172', 178'; IGI 2: 13, 15, 199', 200', 202'; $\rightarrow$ ašar šanîmma

madāru (syllabic) 'to be rotten'

G-stem: ma-di-ra: IGI 2: 196'

mādu, ma'da (syllabic) 'many, very much': IGI 1: 23'; IGI 2: 65', 99', 117’ $\rightarrow$ mēṣa mā du magal (syllabic) 'very much, greatly’: NF 33'

magāru(ŠE.GA) 'to be favourable'

G-stem: magir(ŠE.GA): IGI 1: 183’, mahāru (syllabic) 'to face, oppose, accept'

G-stem: mu-uh/úh-ra: IGI 3: 79', 80', 85'; lim-hu-ra: IGI 3: 85’

Š-stem: tu-šam-har: IGI 3: 31' 
mahru (IGI) 'in front': NF 29' $\rightarrow$ ālik mahri

makūt gabīdi (syllabic) 'pole' of a liver': IGI 3: 81, 89

makūt gabìdi ša imēri (ma-ku-ut ga-bi-di ša ANŠE) “pole’ of a donkey liver’ IGI 3: 75’

makūtu (syllabic) 'pole': $\rightarrow$ makūt gabìdi (̌̌a imēri)

mala (syllabic) 'as much as': IGI 2: 221'

malāku (syllabic) 'to advise':

G-stem: a-ma-lik: IGI 1: 67’

malmališ (syllabic) ‘equally, in equal amounts': IGI 1: 11', 46', 83’; IGI 2: 18, 29, 51', 78',

92', 154', 155'; IGI 3: 53’

malû (syllabic and DIRI(.MEŠ)) 'to be(come) full of'

G-stem: malâ(DIRI-(a/ma)/ DIRI.MEŠ): IGI 1: 23', (malâma) 36', 45’, 68', 71', 77’,

79', 99', 200'; IGI 2: 1, 9, 19, (malâma) 22, 25, 27, 115'-117', (malâma) 121', 139', 207',

213'; IGI 3: 18'; NZa 8'; lim-la-ni-im-ma: IGI 1: 105'; mali(DIRI): IGI 2: 209'

mamma (syllabic) 'somebody', with negation 'nobody': IGI 2: 219'

manna (syllabic) 'whom': IGI 1: 102', 121'

mannu (syllabic) 'who': IGI 1: 159'

manû (ŠID(-nu)) 'to recite'

G-stem: tamannu(ŠID(-nu): IGI 1: 69', 97’, 109', 117’, 144', 154', 186’; IGI 3: 88’; NU

16 ,

maqātu (syllabic) 'to fall, drop'

Gtn-stem: mi-ta-qu-ta-ni: IGI 1: 190’

marāqu (syllabic) 'to grind'

G-stem: ta-mar-raq: IGI 1: 62'; NR 7'

marāṣu (GIG) 'to be sick'

G-stem: marșā(ma) (GIG.(GA)-(ma)): IGI 1: 10', 12', 14', 21', 23', 26', 32', 34', 35', 57',

59', 79', 201'; IGI 2: 117’, 121', 124', 126', 137’?, 204', 216’; IGI 3: 78'; NQ 7’, 8';

marșat(GIG-at): IGI 1: 159'

marat immeri (ZÍ UDU.NÍTA) ‘bile of a sheep': IGI 1: 62'; 'gall-bladder of a sheep':

NR 3'

marat raqqi (ZÍ BAL.GI ${ }^{\mathrm{ku} 6}$ ) 'gall bladder of raqqu-turtle: IGI 2: 71'

marșiš (syllabic) 'bitterly’: IGI 1: 168'

marșu (lúTU.RA) 'sick man': IGI 3: 79', 80’

marșu, (f.) maruštu (GIG.GA(.A)) 'sick': IGI 1: 96', 108', 109', 116', 118', 124', 131', 135',

149', 153', 156', 158', 162', 165', 175', 179', 181'; IGI 2: 185'

mārtu (DUMU.MUNUS) 'daughter': IGI 1: 102', 120', 121'

martu (ZÍ and uzu'ZI) 'gall bladder, bile': IGI 1: 28'; IGI 2: 72’? $\rightarrow$ marat immeri/raqqi

mašāšu (syllabic) 'to wipe off'

G-stem: ta-maš- $a \check{s}^{2}:$ IGI 2: 18

mašku (KUŠ) 'leather bag': IGI 3: 35'?

$\boldsymbol{m} \overline{\boldsymbol{a}} \mathbf{s}(\boldsymbol{t}) \boldsymbol{u}$ ('MAŠ.TAB(.BA)) 'māš(t)u-twin plant': IGI 1: 32'; IGI 3: 97', 99', 102' māšu (MAŠ.TAB.BA) 'a twin': $\rightarrow$ larsīni ša halli lahri ša šumēli ša māšš ūlidu mašmašu (MAŠ.MAŠ) 'mašmašu-healer': IGI 3: 78'-80' 
mašqītu (syllabic) 'potion'; NF 32'

maštakal ((ú)IN.NU.UŠ/ ' IN $6 . U$ UŠ) 'maštakal-plant': IGI 1: 18', 46', 57’; IGI 3: 51'; NF 17',

21'; NU 14' $\rightarrow$ mêe/zèr maštakal

maqātu (syllabic) 'to fall, drop down'

Gtn-stem: mi-ta-qu-ta-ni: IGI 1: 190’

mātu (syllabic and KUR) 'land, earth’: IGI 1: 194'; IGI 2: 217'

mațû (syllabic) 'to diminish (of eye sight)'

G-stem: ma-a-ți: IGI 3: 51'

D-stem: mu-uț-ț: NQ 11'; NS 1'

mayyālu (KI.NÂ) 'bed’: IGI 3: 70’

mazā'u (syllabic) 'to press, squeeze'

G-stem: ta-ma-za-a': IGI 2: 145'

mê bāqili (A SAR.MUNU6) 'maltster-water': NF 34'

mê bīni (A gišŠINIG) 'tamarisk sap': NU 6', 8'

mê būrti (A PÚ) 'well water': NF 21'

mê $\boldsymbol{k} a s \hat{\imath}$ (A GAZI ${ }^{\text {sar }}$ 'sap of a kasû-plant’: IGI 1: 10', 27’, 29’, 52’, 63’; IGI 2: 113'; IGI 3: 31',

72'; NK 12'; NW 2'

mê karān šēlebi (A "'GEŠTIN.KA5.A) 'fox-grape sap’: IGI 2: 100’

mê lišān kalbi (A úEME.UR.GI7) 'sap of dog's tongue-plant’: IGI 2: 100'

mê maštakal (A 'IN 6 .UŠ) 'sap of maštakal-plant': IGI 2: 100'

mê nurmî (A gišNU.ÚR.MA) 'sap of pomegranate’: IGI 3: 47’; NY 7’

mê uhūli qarnānî (A NAGA.SI) 'sap of horned uhūlu-alkali': IGI 2: 91

mer'u $\rightarrow$ merhu

merhu, mer'u (syllabic) 'merhu-kernel’: IGI 1: 197'-199'

mêsallim $\rightarrow$ emesallu

mesû (LUH(-si)) 'to wash'

G-stem: temessi(LUH-(si)): IGI 1: 15’, 22', 52’; IGI 2: 80’, 91’, 119', 153’, 154'; IGI 3: 53’;

NR 8'; NU 6'; imsû(LUH-u): IGI 1: 104 '

mēșa mādu (syllabic) 'completely (lit. little and much)': IGI 1: 67'

mešēltu (na4BAR.DÙ.E) 'mešēltu-whetstone': IGI 2: 159'

mimma (syllabic) 'anything': IGI 1: 193’

minâ (syllabic) 'what': IGI 1: 152'

minâtu $\rightarrow$ minìtu

minitu, (pl.) minâtu (syllabic) 'limbs, physique’: IGI 3: 68', 91'; NT 2’

mirqu (syllabic) 'mirqu-powder?': IGI 3: 41'

mišlu (1/2) 'half': IGI 1: 8', 41', 64'; IGI 3: 50'; NS 7'

mû (A(.MEŠ)) 'water': IGI 1: 15', 22', 31', 63’, 92', 103’; IGI 2: 2, 24, 80', 119', 153', 154', 180'; IGI 3: 34', 71'; NR 8'; NZb 8' $\rightarrow$ mê bāqili/bīni/būrti/kasî/karān šēlebi/lišāan kalbi/maštakal/nurmî/uhūli qarnānî; naqû

muhhu (UGU) 'cranium, brain, top': IGI 2: 77’, 220'; ‘top, upper part, surface': IGI 2: 108', 121', 124' $\rightarrow$ ana/ina muhhi; muhhu ša erî; muhhu ša eșemti kurīti immeri, muhhu ša rīmti, muhhu ša summati zikari 
muhhu ša erî (UGU ša TI ${ }_{8}{ }^{\text {mušen }) ~ ' b r a i n s ~ o f ~ a n ~ e r u ̂-e a g l e ': ~ I G I ~ 2: ~ 205 ' ~}$

muhhu ša eșemti kurīti immeri (UGU ša GÌR.PAD.DU LÚGUD.DA UDU) 'marrow of a short sheep-bone': IGI 3: 46'

muhhu ša rīmti (UGU ša SÚN-tì) 'wild cow’s brain': IGI 2: 77'

muhhu ša summati zikari (UGU ša TU ${ }^{\text {mušn }}$ NITA) 'brain of a male pigeon': IGI 2: 77'

mukīl rēš lemutti (mu-kíl-SAG HUL-ti) ‘supporter-of-evil': NF 26’, 32’; NU 11’ $\rightarrow$ sag-hul-

ha-za

murdinnu, amurdinnu (syllabic) 'murdinnu-bramble (as an eye condition)': IGI 1: 200';

IGI 2: 1, 9

murru ( (sim SES) 'bitter-plant': IGI 1: 86'; IGI 2: 8, 22, 25, 148’?; IGI 3: 102'; NQ 11'; NR 5'; NS 2'; NV 4'

murșu (syllabic) 'illness': IGI 1: 169'

murtappidu (syllabic) 'wandering, roving': NT 5' $\rightarrow$ ețemmu

musukkatu (syllabic and munusú.ZÚG) 'a woman in maternity’ $\rightarrow$ šizbi musukkati

mușa' 'irānu (BIL.ZA.ZA) 'muṣa" irānu-frog': IGI 1: 28' $\rightarrow$ arqu

mūṣu (syllabic) 'mūṣu-stone': IGI 3: 4, 32', 37', 42', 104'; NF 9'; NU 8'

mušālu (syllabic and ŠU.LÚ.ZABAR?) 'bronze palette, mirror’: IGI 2: 74', 75’, 78', 82’

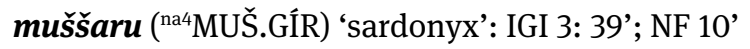

mūšu (GE6) 'night’: IGI 1: 36’; IGI 3: 28', 73’, 74'; NF 28', 31’

$\mathbf{N}$

nabalkutu (BAL-at) 'to cross over, change sides'

N-stem: tabbalakkat(BAL-at) 'you transfer': IGI 2: 86'

nabāsu ('íg HÉ.MED/ME.DA) 'red wool': IGI 1: 39'; NZ 12'

nablu (syllabic) 'flame, meteor': IGI 1: 190'

$n \bar{a} d u$ (KUŠ.EDIN) 'waterskin': IGI 1: 12'

nadû (syllabic and ŠUB) 'to put, apply, cover, set, install, recite, cast out (an

incantation), to drip tears'

G-stem: tanaddi(ŠUB(-di)): IGI 1: 20', 72'; IGI 2: 2, 73', 74', 134'; IGI 3: 30', 34',

(tanaddìma) 35', 54', (tanaddìma) 95'; NF (tanaddìma) 21'; NU 5'; nadâ(ŠUB-a): IGI

1: 92'; it-ta-di: IGI 1: 93'; i-di-ma: IGI 1: 171', 192'; li-in-na-di: NF 28'

Gtn-stem: tattanaddi(ŠUB.MEŠ): IGI 1: 12'; tattanaddǐ̌u (ŠUB.ŠUB-šú) IGI 3: 71';

tattanaddi(ŠUB.ŠUB-di): IGI 2: 78'; NF 29'; ittanaddâ(ŠUB.ŠUB-a): NK 10'; NQ 5',

10'; NS 1'

naglabu (syllabic and GÍR.ŠU.I) ‘barber’s knife, scalpel’: IGI 1: 190’; IGI 2: 201’, 203’ $\rightarrow$ șurru

nâhu (syllabic) 'to come to rest, appease, be still'

G-stem: li-ni-ih-ka: NU 16'

D-stem: a-na nu-úh-hi: IGI 2: 193'

nakāpu (syllabic) 'to push away' 
D-stem: ú-na-kap: IGI 1: 80'

nakāsu (KUD) 'to cut'

G-stem: tanakkis(KUD): IGI 1: 38'

nakkaptu (SAG.KI) 'head-temple': IGI 1: 12', 13', 29', 39', 69', 144', 146', 148', 154', 161',

186'; NV 6'; NZa 8', 10' to

naklu (syllabic) 'elaborate': IGI 2: 220'

nâlu (NÂ-al) 'to lie down' $\rightarrow$ itūlu

Š-stem: 'to pickle, cure': tušnâl(NÁ-al): IGI 2: 71'; NR 3'

namāru (ZALAG.GA) 'to be(come) bright, shine'

G-stem: namrā(ZALAG.GA): IGI 2: 204'

namru (syllabic) 'bright, clear, shining': IGI 2: 218'; IGI 3: 79', 86'

napāhu (syllabic and BÚN, MÚ(-ah)) 'to blow; to light up, ignite'

G-stem: tanappah(BÚN): IGI 1: 7', 86'-88'; IGI 2: 115'; tanappah(MÚ-ah): IGI 1: 56';

IGI 2: 132', infinitive: ina napāhi 'at sunrise': NZb 11': nap-hat: NV 6'

D-stem: nu-up-pu-ha: IGI 2: 56'; nuppuhāma(MÚ.MEŠ-ma): NK 6'

napāšu (syllabic) 'to ease off, to breathe'

G-stem: na-pa-še: IGI 1: 94’

napharu (PAP) 'total': IGI 2: 9; IGI 3: 34'

napšalti lubki (syllabic) 'softening ointment': IGI 3: 101' $\rightarrow$ lubku

napțu (İ.KUR.RA) 'naphtha': NV 3'

napû (SIM) 'to sift'

G-stem: tanappi (SIM): IGI 2: 56’; IGI 3: 72’

naqû (BAL-qí) 'to pour out (tears, water)'

G-stem: dimta inaqqi(BAL-qí): NV 6'; naq me-e: NZb 8'; i-naq? -qi?: NZb 8' $\rightarrow$ dimtu,

$m \hat{u}$

narmaktu (syllabic) 'bath': NF 32'

nāru (syllabic and 'ID) 'river': IGI 1: 113', 188',

nasāhu (syllabic and ZI) 'to tear out, remove'

G-stem: inassah(ZI): IGI 2: 5; nasāhi(ZI): IGI 2: 124'; inassaha(ZI-ha): IGI 2: 138'; i-

na-sah: IGI 2: 176'; ana nasāhīšu(ana ZI-šú): IGI 3: 11; ina nasāhī̌̌u(ina ZI-šú): NZa

10 '

N-stem: innassah: NY 4'

nașābu (syllabic) 'to suck'

G-stem: i-na-șab: IGI 1: 75'

našāku (syllabic) 'to bite'

G-stem: na-aš-ku: IGI 2: 111’ $\rightarrow$ kalīt lalî lakî ša šamma lā našku uțtata lā șabitu

- nâši (syllabic, 1 pl. dat. pron.) 'for us': IGI 1: 178'

našû (syllabic and ÍL) 'to carry, lift, + ventive to bring, carry along, pick up'

G-stem: liš-šá-(a)-ni: IGI 1: 103’, 122'; ta-aš-šâ-ni: IGI 1: 187’; taš-ša-ni: IGI 1: 189';

áš-ši: IGI 1: 192'; inašši(ÍL-(ši)): IGI 3: 70', (inaššìma) 78'

natāku (syllabic) 'to drip

D-stem: tu-na-tak/ták: IGI 1: 18', 48’; IGI 2: 76’, 89’, 148’, 158’, 169’, 175’ 
națālu G (IGI-al) 'to see'

G-stem: inatțal(IGI-al) IGI 1: 61'; ina-ța-la: IGI 3: 49’; NS 6’; i-na-aț-ṭa-la: IGI 3: 66’;

na-ța-la: NQ 10'; NS 1'

nawāru $\rightarrow$ namāru

nemsētu (syllabic) 'tub’: IGI 2: 111'

nêšu (syllabic) 'to get better'

G-stem: ina-eš: IGI 1: 25’, 26’, 118’, 154’, 181’, 186’; IGI 2: 7; IGI 3: 6, 90’; NF 12’, 16’;

NY 7a'

nēšu (UR.MAH) 'lion’ $\rightarrow$ lipi nēši

- ni (syllabic) 'us': IGI 1: 178'

- ni (syllabic) 'me': IGI 1: 74'

nignakku (NÍG.NA) 'incense burner': IGI 3: 77'

nikiptu (syllabic) 'nikiptu-plant': NU 2' $\rightarrow$ zikaru, sinnišțu

nikiptu zikar u sinniš (ni-kip-tu 4 NÍTA $u$ MUNUS) 'male and female nikiptu-plant': IGI

1: 46'; NF 18'

niksu (syllabic) 'cut off': IGI 1: 91'; NW 4'

- ni $(m) \rightarrow-a(m)$

nīnû ('KUR.RA) 'nīnû-mint': IGI 1: 32’, 61'; IGI 2: 22, 25; IGI 3: 41', 97’, 99’, 102'; NK 3’;

NQ 12'; NR 6'; NS 3'

niqqu (syllabic) 'fig seed': IGI 1: 114'

niqû (UDU.SISKUR) 'offering': IGI 3: 45'

Nisannu $\rightarrow$ Month Names

nisqu (syllabic) 'high level': IGI 2: 218'

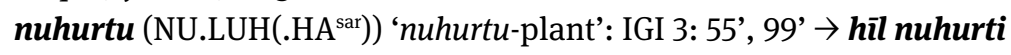

nurmû (gišNU.ÚR.MA) 'pomegranate' $\rightarrow$ hașab/qilip/mê nurmî

nūru (syllabic) 'lamp(-light)': IGI 2: 90’; IGI 3: 9

\section{$\mathbf{P}$}

pa'āṣu (syllabic) 'to crush'

G-stem: ta-pa-a-aṣ: IGI 2: 102’

D-stem: tu-pa-áṣ: NZa 10'

pa'șu (syllabic) 'crushed': IGI 2: 96'

pahāru (syllabic) 'to bring together, gather'

D-stem: ú-pa-har: IGI 1: 197’; ina pu-hu-ri-šú: IGI 1: 197’; tu-pa-har: IGI 3: 82’

palāsu (syllabic) 'to notice'

N-stem: ip-pa-lis-su-ma: IGI 1: 151'

pānu (IGI, IGI.MEŠ) 'front, face'; 'top': IGI 2: 64'; NZa 8'; 'in front': NF 19'; pāna pāni:

IGI 1: 59'; $\rightarrow$ ana pān, ikil pāni, ina pāni, pānūšu ișșanundū ( $\rightarrow$ șâdu), șulum pāni,

šār pāni, šatti pāna

papparhû (BABBAR.HI ${ }^{\mathrm{sar}}$ ) 'papparhû-purslane': IGI 1: 19' 
parāku (syllabic) 'to block, lie across, park'

G-stem: pa-rik: IGI 1: 101', (parikma) 126'

parāsu (KUD) 'to cease, stop'

G-stem: paris(KUD-is): NW 4'

N-stem: ipparras (KUD-as): NZ 3'

parzillu (AN.BAR) 'iron'; NT 9'

passu (ZA.NA) 'collyrium stick (lit. game disk)': IGI 1: 63'; IGI 2: 24; IGI 3: 53'

pašāru (syllabic) 'be released, saved':

N-stem: na-ap-ši-ir: IGI 1: 66'

pašāšu (ŠÉŠ) 'to smear, anoint'

G-stem: tapaššaš(ŠÉŠ(-aš)) 'you smear’: IGI 2: 79'; IGI 3: 35’, 37’, 39’;

(tapaššassuma(ŠÉŠ-su-ma)) NF 20’, 23’; NU 5’; tapaššassu(ŠÉŠ-su): NV 3' patālu, petēlu (syllabic) 'to twist'

G-stem: te-pe-til: IGI 1: 160’, patānu (syllabic) 'to eat' $\rightarrow l \bar{a}(m)$ patān

patāqu 'to build (a mud wall)'

G-stem: pat-qat: IGI 1: 93', 120', 127'

patar siparri (GÍR.ZABAR) ‘bronze knife’: IGI 1: 14’, 38’; IGI 2: 72’, 79’, 195’

pațāru $\left(\mathrm{DU}_{8}\right)$ 'to loosen'

G-stem: tapațtaršuma(DU8-šú-ma) IGI 1: 38'; IGI 2: 7; pu-țur: IGI 1: 130'; lā

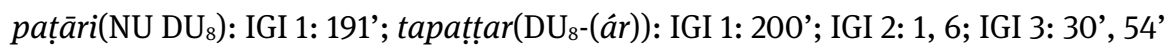

pel äribi (NUNUZ UGA ${ }^{\text {mušen }) ~ ' r a v e n ' s ~ e g g ': ~ I G I ~ 2: ~ 79 ' ~}$

pelû (NUNUZ) 'egg' $\rightarrow$ pel āribi

pēmtu (NE) 'charcoal': IGI 1: 33’; IGI 2: 74’; IGI 3: 64', 100', 103'; NK 5’; NU 8'; NY 4' peșû (BABBAR) 'white': $\rightarrow$ dišpu peșû, iškūru peșû, šammu peșû, šīpātu peșâtu petēlu $\rightarrow$ patālu petû (BAD-(te)) 'to open'

G-stem: ipetti(BAD): IGI 1: 23’; IGI 2: 117'; tepette(BAD-te) 84'; i-pet-tu: NF 30’ pillû zikaru ( ${ }^{\text {išs }}$ AM.TAR NÍTA) 'male pillû-plant': IGI 2: 130' $\rightarrow$ arti pillî zikari piqannu (A.GAR.GAR) 'droppings' $\rightarrow$ piqan șabìti piqan șabīti (A.GAR.GAR MAŠ.DÀ) 'gazelle droppings’: IGI 2: 177’; IGI 3: 72' pitiltu (ŠU.SAR) 'string': IGI 1: 76' pitiqtu (syllabic) 'a mud wall’: IGI 1: 93', 120', 127’, pû (syllabic and KA) 'mouth, opening': IGI 1: 160'; NY 7a' pursimìtu $\rightarrow$ pursìt dāmi pursindu $\rightarrow$ pursìt dāmi pursītu ( ${ }^{\mathrm{dug}}$ BUR.ZI) 'pursītu-bowl': IGI 1: 3'; IGI 2: 156' $\rightarrow$ pursìt dāmi pursìt dāmi (pur-si-it / pur-si-in-di / pur-si-mi-it MÚD / da-a-mi) 'blood vessel in the eye':

IGI 1: 91', 112', 136', (pursindi dāmi) 176', (pursimìt dāmi) 187' pūṣu (BABBAR) 'white spot': IGI 2: 124' pūtu (SAG.KI) 'forehead': IGI 3: 8'; NF 46' 


\section{Q}

qablu (MURUB ${ }_{4}$ ) 'middle': IGI 1: 180'

qabû (syllabic and $\mathrm{DUG}_{4 . \mathrm{GA}}$ ) 'to say, speak'

G-stem: taqabbi(DUG 4 .GA): IGI 1: 31'; qấ-bu-ú šá: IGI 1: 100', 134'; i-qab-bi: IGI 3:

79', 80'; NF 14'; i-qab-bu-u: IGI 3: 82', 83'; liq-bi; NF 13', (NT 4')

qadūtu šikāni (IM.GÚ.EN.NA) 'river silt': IGI 2: 8

qalû (syllabic, BIL-la, BIL-lu, BíL,) 'to roast'

G-stem: ta-qal/qàl-lu: IGI 1: 33’; IGI 2: 111’, 112'; IGI 3: 103’; taqallu(BÍL): IGI 2: 198’;

taqallu(BíL-lu): NK 7'; taqallu(BIL-lu): NZ 24'

qalû (BÍL) 'roasted': IGI 2: 68'

qanānu, kanānu (syllabic) 'to roll up'

D-stem: ú-qa-an-na-an: IGI 1: 75’

qanû (GI) 'reed' $\rightarrow$ gūra ša qanî, lipi qanî, qanû țābu

qanû țābu (GI DÜG.GA) 'sweet reed': IGI 2: 113'

qaqqad șurāri (SAG.DU EME.ŠID) 'lizard's head': IGI 2: 74'

qaqqadu (SAG.DU, SAG) 'head': IGI 1: 8', 23', 24'; IGI 2: 6, 7, 117', 118'; NV 1'; NZ 19' $\rightarrow$

qaqqad șurāri

qarnu (SI) 'horn' $\rightarrow$ uhūlu qarnānû

qatāru (syllabic) 'to fumigate'

G-stem: tu-qat-tar-šu: NK 5'; tuqattaršuma(SAR-šú-ma): NY 4'

$q \bar{a} t u$ (syllabic and ŠU) 'hand': IGI 1: 104', 160', 173'; IGI 2: 80'; IGI 3: 66', 88', (qāt

ummâni) 96', (qāt asî) 105' $\rightarrow$ asû; qāt Ištar/Šulpaea/Adad rāhiș; ummânu qāt Ištar (ŠU ' 15 , 'I $\check{s}_{8}$-târ) 'hand of Ištar': IGI 2: 63', 66'

qāt Adad rāhiṣ (ŠU dIŠKUR ra-hi-iș (gloss of Šulpaea)) 'hand of Adad, the rain maker':

IGI 2: 62'

$q \bar{a} t$ Šulpaea (ŠU d Šul-pa-è-a) 'hand of Šulpaea': IGI 2: 62'

qatû (TIL.LA) 'to come to an end, finish'

D-stem: uqatta(TIL.LA): IGI 1: 70’

qēm aban suluppī (Zİ NA 4 ZÚ.LUM.MA) 'powder of date stones': IGI 1: 27’

qēm ašnan (Zİ dŠE.TIR) 'cereal flour': IGI 2: 52'

qēm buqli (Zİ MUNU 6 ) 'malt flour': NZ 22'

qēm burāši (ZI İimLI) 'powder of juniper': NK 11'

qēm hallūri (Zİ GÚ.GAL) 'chickpea powder': IGI 1: 29'; IGI 2: 68'; NK 10', 13'

qēm kakkî (Zİ GÚ.TUR) 'powder of lentil': IGI 2: 68'; NK 10', 13'

qēm kasî (ZÍ GAZI ${ }^{\mathrm{sar}}$ ) 'powder of kasû-plant': IGI 1: 29'; IGI 2: 68'; NK 7', 12'

qēm kibti (Zİ GIG) 'wheat flour'; NZ 15', 17'

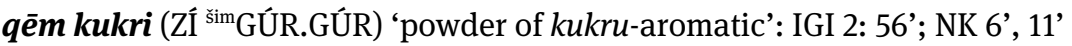

qēm labti (Zİ ŠE.SA.A) 'flour of roasted grain': IGI 1: 200'; IGI 2: 1, 11, 56'; IGI 3: 72'; NK

7', 11'

$\boldsymbol{q} \bar{e} \boldsymbol{m}$ suādi (ZI İ $\left.{ }^{\mathrm{sim}} \mathrm{MAN} . \mathrm{DU}\right)$ 'powder of suādu-aromatic': NK 12'

$\boldsymbol{q} \bar{e} \boldsymbol{e}$ šunê (Zİ giššE.NÚ.A) 'powder of šunû-tree': NK 11' 
qēmu (Zİ) 'flour, powder' $\rightarrow$ qēm aban suluppī, qēm ašnan, qēm hallūri, qēm labti, qēm kasî, qēm kukri, qēm šunê, qēm burāši, qēm buqli

qerbu (syllabic) 'interior, inside': IGI 2: 222'

qerēbu (syllabic) 'to come close:

D-stem: li-qer-ri-bu: IGI 1: 76'

qeršu (syllabic) 'stripe of meat': IGI 2: 3

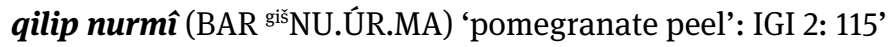

qilip šūmī (BAR SUM ${ }^{\text {sar }}$ ) 'peel of garlic': NU 14'

qilpu (BAR) 'skin of plant, fruit, peel' $\rightarrow$ qilip nurmî/šūmī

qištu (NÍG.BA) 'fee’: IGI 1: 95', 107'

qudru ('KI. ${ }^{\mathrm{d} I S ̌ K U R) ~ ' q u d r u-p l a n t ' ~} \rightarrow$ zēr qudri

qūqānu, gūqānu (syllabic) ‘qūqānu-worm': IGI 2: 27

qutāru (syllabic) 'fumigation': IGI 3: 8

qutru (syllabic) 'fume, smoke’: IGI 2: 90', 135'

\section{$\mathbf{R}$}

rabāku (syllabic) 'to make an infusion'

G-stem: tara-bak: IGI 1: 78', 200’; IGI 2: 1, 56’, 63’, 68', 84’, 99'; NK 7'

rahāșu (syllabic) 'to make rain, drench' $\rightarrow$ tahāhu

G-stem: ra-hi-iș: IGI 3: 62'

Gtn-stem: ir-ta-na-haș-ma: NF 35'

rakāsu (syllabic tara-ká/às, and KEŠDA) 'to bind, bandage body parts'

G-stem: tarakkas(KEŠDA): IGI 1: 8’, 109', (tarakkasma) 118', (tarakkasma) 154',

(tarakkasma) 181', (tarakkasma) 186'; IGI 2: 2, 3, 188'; tarakkassu(KEŠDA-su): IGI 1:

97'; 144'; NF 46'; tara-ká/às: IGI 1: (tarakkasma) 39', 59', 69'; ru-kus-ma: IGI 1: 172';

tar-ku-si: IGI 1: 178'

ramāku (syllabic and $\mathrm{TU}_{5}$ ) 'to bathe, soak'

G-stem: tara-muk: IGI 1: 81'; tarammukšu(TU ${ }_{5}$-šú): NF 19'; $\operatorname{rammuk}\left(\mathrm{TU}_{5}\right)$ : NU 6' ramānu (syllabic) 'self': IGI 1: 168'

rapādu ('ra-pa-di) 'plant for rapādu-disease' $\rightarrow$ šuruš šammi rapādi

rapšu (syllabic and DAGAL(-te/i)) 'wide, great’: IGI 1: 104'; IGI 2: 217' $\rightarrow$ tâmtu

raqqu(BAL.GI ${ }^{\mathrm{ku} 6}$ ) 'raqqu-turtle' $\rightarrow$ marat raqqi

rašû (TUK) 'to acquire, to develop (a disease)'

G-stem: iraššâ(TUK-(a)): IGI 1: 145’; NV 1'; zūta irašši(TUK): NF 34' $\rightarrow$ zūtu

rebiat (IGI.4.GÁL.LA) 'one-fourth': IGI 2: 84'; IGI 3: 50'; NS 3', 7'

rebû (4) 'fourth': IGI 2: 2, 7

rēš īnī (SAG IGI.MIN) 'upper part of the eyes or eyelids': IGI 1: 84'

ribku (syllabic) 'mixture, infusion': IGI 1: 33'; IGI 2: 7, 99'; IGI 3: 43', 98', 103'

rikibti arkabi (U $\left.\mathrm{U}_{5 .} \mathrm{ARGAB}^{\text {mušen }}\right)$ 'bat guano': IGI 1: 6', 34', 41', 44', 61', 64'; IGI 2: 8, 23,

26, 92', 122', 140', 141', 144', 152', 197'; IGI 3: 49'; NQ 11'; NS 2', 6’ 
riksu(KEŠDA) 'binding': IGI 2: 5; IGI 3: 60'

rīmtu (SÛN) 'wild cow': $\rightarrow$ muhhu ša rīmti

rūqu (syllabic and SÙ.UD.DA) 'distant': IGI 1: 164'; IGI 3: 9

rūštu (SAG) 'top quality, premium': $\rightarrow$ šaman rūšti, šikar rūšti

ru'tìtu (ÚH-díD) 'ru'titu-sulphur (lit. river spittle)': IGI 3: 94'

ru'tu (ÚH) 'spittle': IGI 1: 47'

\section{$\mathbf{S}$}

sāabu ( $\left.{ }^{\mathrm{na} 4} \mathrm{~s} a-a-b u\right)$ 'sābu-stone'; NT 10'

sahlânu (syllabic) 'sahlânu-plant': IGI 2: 122' $\rightarrow$ sahlû

sahlû (syllabic and ZÀ.HI.LI.(A) (sar) 'sahlû-plant': IGI 1: 9', 15', 29', 32', 35', 51', 200'; IGI

2: 1, 6, 22, 26, 65', 68', 98', 191'; IGI 3: 55', 94', 97’, 99', 104', 107'; NK 3’, 14'; NQ 8';

NR 6'

sâku (SÚD and syllabic) 'to pound'

G-stem: tasâk(SÚD): IGI 1: 6', 7', 9'-11', 13'-16', 18', 20', 22', 27', 34', 35', 38', 40',

41', 42', 43'-47', 54', 55', 58', 60', 64', 72', 78', 82', 87', 88'

IGI 2: 4, 16, 18, 20, 21, 23, 24, 29, 50'-53', 55', 58', 60'-62', 64', 66', 67', 69'-72', 74'-

77', 79', 83'-88', 93', 97', 99', 101'-104', 107', 108', 113', 115', 116', 119', 123', 125',

127', 129', 132', 133', 139', 140'-144', 146'-148', 154', 155', 157'-159', 161', 162', 164',

165', 167'-169', 173', 180', 183', 184', 186', 187', 195', 205'

IGI 3: (tasâkma) 6, 12, 38'-40', 48', 50', 53’, 59', 65', 67', 95’, 100', 103'; NF 5',

(tasâkma) 12', 16'; NP 2'-5'; NQ 12'; NR 2', 4', 5'; NS 4', 8'; NU 3', 5', 7', 9'; NV 2'-4';

NW 6'; NZ 2'

ta-sàk: IGI 1: 16', 19', 25’; IGI 2: 87’, 107’, 178', 186’

salā'u, šalā'u (syllabic) 'sprinkle water'

G-stem: lis-la-ni-im-ma: IGI 1: 105'

samānat (8) 'eight': IGI 1: 33'; IGI 2: 9, 138'

sāmtu ( $\left.{ }^{\mathrm{na}} \mathrm{GUG}\right)$ 'carnelian': NF 9'

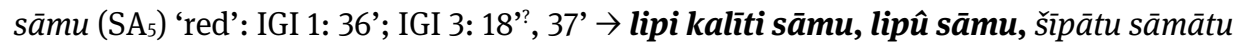
sanāqu (syllabic) 'to check, execute'

G-stem: sa-niq: IGI 1: 100', 134'; as-niq: IGI 2: 221’

sapānu (syllabic) 'to dissolve, soften, flatten'

G-stem: ta-sa-pan: IGI 1: 63'; NR 7’

sâpu (syllabic) 'to draw water'

G-stem: li-sa-pa-a-ni: IGI 1:103’

Gtn-stem: li-is-sa-pa-ni-im-ma: IGI 1: 123'

sāqu (syllabic) 'thigh' $\rightarrow$ lipi sāqi șabīti

sarāmu (syllabic) 'to cut'

G-stem: ta-sa-rim: IGI 2: 90’ 
sebe (7) 'seven’: IGI 1: 97’, 109’, 144'; IGI 2: 28, 59’, 95’, 103’, 138’; IGI 3: 27’, 54’, 78’, 87’, 102'; NF 44', NS 3'; NU 14'

sebet $u$ sebet (7 u 7) 'seven and seven': IGI 1: 117', 154', 186'

sekēru (ÚŠ) 'to heat'

G-stem: tesekker(ÚŠ-ker/er): IGI 3: 71'; NF 35'

sekru (syllabic) 'warmed-up': IGI 2: 113'

sêru (syllabic) G-stem 'to smear, spread onto'

G-stem: te-ser: IGI 1: 12'

sīhu ( gišsi-ha) 'sīhu-artemisia': IGI 1: 19'; NV 7'

sikillu ('SIKIL) 'sikillu-plant': NF 18', 45'

sikkat șēli immeri (GAG.TI.TI UDU) 'lamb chop': IGI 2: 75'

sīkti labti (si-ik-ti ŠE.SA.A) 'powder of roasted grain': IGI 2: 146', 206'

sillurmû, Sîn-lurmâ (syllabic and d30-lu-ur-ma-a) 'night/day blindness': IGI 3: 73'-75', 81 '

simmu (syllabic) 'wound, sore': IGI 1: 163', 165'

Sîn-lurmâ $\rightarrow$ sillurmû

sinništu (MUNUS) 'female': IGI 1: 46’, 67'; NF 18', 27' $\rightarrow$ nikiptu

siparru (ZABAR) 'bronze' $\rightarrow$ patar siparri, uppi siparri

suādu (šmMAN.DU) 'suādu-aromatic': IGI 2: $2 \rightarrow \boldsymbol{q} \bar{e} \boldsymbol{m}$ suādi

suluppu (ZÚ.LUM.MA) 'date’: IGI 3: 22', 51' $\rightarrow$ qēm aban suluppī

summatu (TU ${ }^{\text {mušen }}$ ' 'pigeon': $\rightarrow$ muhhu ša summati zikari

sumuktu (syllabic) 'sumuktu-dirt': IGI 1: 139'

supālu (syllabic and úZA.BA.LAM) 'supālu-juniper': su-pa-la: IGI 1: 21'

sūqu (SILA) ‘street': IGI 1: 138’, 188’

\section{S}

șabātu (syllabic and DAB) 'to seize, grasp, afflict'

G-stem: șa-bi-tú: IGI 2: 111'; iṣbassu(DAB-su): IGI 3: (ișbassuma) 9, 10; NF 26', 32’;

NU (ișbassuma) 12'; NZa (ișbassuma) 9'

șabìtu (MAŠ.DÁ) 'gazelle' $\rightarrow$ lipi kursinni șabīti, lipi sāqi șabīti, piqan șabīti, lipi

eșemti șabīti

șâdu (syllabic) 'to turn around, with pānu 'to have a vertigo'

Gtn-stem: pānūšu i-șa-nun-du: NZa 9'

șahātu (SUR(-at)) 'to press out, draw liquid into vessel'

G-stem: taṣahhat(SUR(-at)): IGI 1: 3', 81'; IGI 2: 156'; NU 7'

D-stem: șu-uh-hu-ta: IGI 2: 63'

șalālu (syllabic and NÂ) 'to sleep'

G-stem: i-șa-lal-ma: IGI 1: 36'; iṣallal(NÁ): IGI 3: 68'

șalāpu (syllabic) 'to cut open, dissect'

G-stem: ta-șa-lip: IGI 1: 28' 
Št-stem: šu-te-eș-li-pa-a-tu: IGI 1: 112’ șallāmtu (MUŠ.GE6) 'black snake' $\rightarrow$ lipi șallāmti șallu (syllabic) 'sleeping, resting': NF 31' șalmu (GE6) 'dark, black': IGI 3: 40’ $\rightarrow$ dām kurșipti alpi șalmi, lipi kalit alpi șalmi,

șurru șalmu

șamādu (LAL(-id)) 'to bandage'

G-stem: tașammid(LAL(-id)): IGI 1: 10', 13', 15’, 18', 19', 22', 29', 35', 37', 48', 78',

200'; IGI 2: 1, 4, 6, 56', 68', 145', 181'; IGI 3: 56', 72'; NK 7’, 12', 15'; NP 6’; NV 5’; NZ

5', (6', 7'), (tașammidma) 10'

G-stem: tașșanammid(LAL.MEŠ): IGI 2: 114'

D-stem: ú-șa-am-mad': NZa 10'

șehru ((lú)TUR) 'a chick': IGI 2: 106'; 'a child': IGI 3: 82'

șētu (UD.DA) 'sun-heat’: IGI 1: 20'; IGI 2: 9; IGI 3: 49’; NS 6’

șillû ('sišDÁLA) 'needle’: IGI 2: 105', 166'

șillu (syllabic and GISSU) 'shadow/film (on/in the eye)': IGI 1: 23', 80', 92'; IGI 2: 117',

121', 199', 200', 202', 207', 209'

șimdu (syllabic) 'bandage': IGI 1: 76'

șirihtu (syllabic) 'inflammation': IGI 1: 105'; IGI 2: 209'

șit šamši ('UTU.È) 'sunrise': IGI 3: 29'

șubātu (TÚG) 'cloth': IGI 2: 28, 174'; NF 23'

șulum pāni (syllabic) 'dark of face, anger’: IGI 1: 130'

șumlalû ('sim GAM.MA) 'ṣumlalû-aromatic'; NZ 9'

șupru (UMBIN) '(toe-)nail’: IGI 2: 220’

șurār igāri (EME.ŠID É.GAR 8 ) 'wall-lizard': IGI 2: 74'

șurāru (EME.ŠID/DIR) 'lizard' $\rightarrow$ qaqqad șurāri, șurār igāri, zê șurāri

șurru (syllabic and na4ZÚ) 'flint (knife)': IGI 1: 190'; $\rightarrow$ șalmu, naglabu

șurru șalmu ( ${ }^{\mathrm{na}}{ }^{2} \mathrm{ZU}$ GE 6 ) 'black flint = obsidian': IGI 3: 40’

\section{$\check{\mathbf{S}}$}

ša (syllabic) 'her, its': IGI 1: 104', 114', 121', 173'

ša (indirect genitive particle) 'of, from': IGI 1: 6', 33', 61', 92', 100', 102', 105', 113', 114', 121', 134', 146', 148', 188', 191', 193', 199'; IGI 2: 10, 11, 18, 26, 27, 57', 77', 90', 108', 124', 135'; IGI 3: 96', 98', 101', 103'; NF 13', 14'; NK 8'; NR 5'; NZ 11'; NZa 9'; 'because': IGI 1: 137': 'regarding': IGI 1: 166'; 'against': IGI 3: 32', 43', 46' $\rightarrow$ baltu ša țābti, dāmu ša libbi šahî, gūru ša qanî, gurušgaraš ša šahî, kalìt lalî lakî ša šamma lā našku utțata lā șabitu, larsīni ša halli lahri ša šumēli ša māšī ūlidu, kammu ša aškāpi, makūt gabīdi ša imēri, muhhu ša erî, muhhu ša eșemti kurīti immeri, muhhu ša rīmti, muhhu ša summati zikari, ša libbi, šuhtu ša aškāpi, šuhtu ša tangussi 
ša (syllabic) 'which, (the one) who, whose, whatever, what': IGI 1: 83', 97', 99', 104', 109', 152', 166', 169', 174’; IGI 2: 62', 64', 106', 217’, 219'; IGI 3: 78', 83’’; NU 8'; NW 4'

ša libbi (ša ŠÀ) 'from out': IGI 1: 199'

šadādu (syllabic) 'to extend, stretch (over body parts)'

G-stem: šad-du-ma: IGI 3: 70’

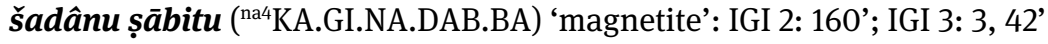

šadû (IM.3) 'east wind': IGI 1: 115'

šadû (KUR-(ú)) 'mountain': IGI 1: 101', 126' $\rightarrow$ dišpu/uqni šadê

šaharru, šuharru (syllabic) 'porous': IGI 1: 91', 136', 176', 187'

šahātu (syllabic) 'to rinse'

G-stem: ta-áš-hu-tu: IGI 1: 83’

šahhû (túgŠÀ.HA) ‘šahhû-cloth': IGI 3: 76’, 77’

šahû (ŠAH) 'pig’: IGI 1: 74' $\rightarrow$ dāmu ša libbi šahî, gurušgaraš ša šahî, zappi šahî

šakāku (syllabic and È-ak) 'to thread (on a string); to twist'

G-stem: ta-šak-kak: IGI 3: 76'; tašakkak (È-ak): NF 44'; NU 13’

šakānu (syllabic and GAR(-an)) 'to put, place, present'

G-stem: tašakkan(GAR-(an)): IGI 1: 59', 84'; IGI 2: 71', 74', 75’, 78', 82', 119', 157'; IGI

3: (tašakkanma) 35', 64', 76', 77’, (tašakkanma) 88'; NS 4'; NU 4', 10';

tašakkanu(GAR-nu): IGI 3: 93'; NU (tašakkanuma) 8'; šak-na: IGI 1: 61'; taš-ku-ni:

IGI 1: 76'; iš-ta-kan: IGI 1: 163', 165'

Gtn-stem: ištanakkan(GAR.GAR-an): IGI 3: 10

šalā'u $\rightarrow$ salā'u

šalāmu (SILIM) 'to be safe'

G-stem: išallim(SILIM-im): IGI 3: 35'; i-šal-lim: IGI 3: 36'

šalāš (3) 'three': IGI 1: 64', 78'; NZb 2'

šalāšišu (3-šú) 'three times': IGI 1: 24', 31'; IGI 2: 2, 3, 5, 118', 144'; NF 13'; NU 6' $\rightarrow$ ištiššu

šinī̌su šalāšišu

šalmu (syllabic) 'healing, healthy': $\rightarrow$ tēqìtu šalimtu

šalû(syllabic) 'to emerge'

G-stem: i-šal-lu: IGI 2: 64'

šamāhu (syllabic) 'to grow, flourish'

G-stem: šam-ha: IGI 2: 199’; šamhat': IGI 2: 200', 202’

šaman asi (I İim GÍR) 'oil of asu-aromatic': NF 20'

šaman ballukki (I šmBAL) 'oil of ballukku-aromatic': NF 22'

šaman erēni (İ(+GIS) EREN) ‘cedar oil’: IGI 2: 24, 51’, 85’, 123’, 125’, 194’; IGI 3: 48’

šaman ișșūr hurri labīri? (İ.GIŠ MUŠEN.HABRUD LIBIR.RA) 'fat from an old

partridge?': IGI 3: 42'

šaman kurkî (İ KUR.GI ${ }^{\text {mušen }) ~ ' g o o s e ~ f a t ' ~ I G I ~ 3: ~ 40 ' ~}$

šaman pūri (İ.GIŠ.BUR) 'pūru-oil': IGI 2: 99'

šaman rūšti (İ.GIŠ.SAG) 'first class sesame oil': IGI 1: 83'

Šamaš $\rightarrow$ see Divine Names 
šamaškillu (SUM.SIKIL) 'šamaškillu-onion': IGI 1: 26'

šamātu (syllabic) 'to tear down, strip away'

G-stem: ta-šam-ma-aț: IGI 2: 95'

šammi aš̂̂ (Ú $a$-ši-i) 'ašû-disease-plant (against 'ašû-disease')': IGI 1: 51'; IGI 2: 192'; IGI 3: 41', 94'; NQ 3', 12'; NS 3'

šammu (Ú) 'plant, drug': IGI 1: 33’, 62’, 64’; IGI 2: 7, 9, 26, 57’, 106', 109', 123’; IGI 3: 5,

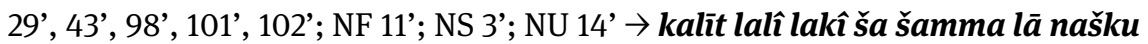
utțata lā șabitu, šammi ašî, šammu ēdu, šammu peṣ̂, šuruš šammi rapādi šammu èdu (Ú.DILI) 'single plant'; NU 3', 14'

šammu peșû (Ú.BABBAR) 'white plant': IGI 1: 22', 34', 41', 42', 44', 56', 58', 61', 82', 86', 87', 88'; IGI 2: 8, 10, 23, 25, 57', 58', 66', 70', 73', 92', 113', 114', 122', 145', 196'; IGI 3: 50', 52', 104'; NK 8'; NQ 11'; NR 6'; NS 2', 7'; NV 2' $\rightarrow$ hașbi šammi peșî

šamnu (İ(.GIŠ) 'oil': IGI 2: 61', 62', 71', 74', 86', 88', 93', 116', 162', 184', 193'; IGI 3; NF 5'; NU 2', 9'; NV 2'; NW 6' $\rightarrow$ šaman asi, šaman ballukki, šaman erēni, šaman ișșūr hurri labīri', šaman kurkî, šaman pūri

šamnu (İ.GIŠ, İ+GIŠ) 'sesame oil': IGI 1: 8', 12', 26’, 44', 54’; IGI 2: 64’, 73', 77’, 84’; IGI 3: 35', 83', 100'; NU 5'; NV 2'; NZ 2', 21' $\rightarrow$ rēštû

šamû (syllabic and AN(-e)) 'sky/heaven': IGI 1: 102', 121', 163', 164'

šanā'u (syllabic) 'to block (off)'

G-stem: še-en- $a$ : IGI 1: 91’

D-stem: šu-un-nu-'a: IGI 1: 40'

Dt-stem: ul-ta-ta-ni-'a: IGI 1: 79'

šanîš (MIN) 'alternatively’: IGI 1: 87’, 88', 183’; IGI 2: 140', 141'; 'ditto': IGI 2: 13, 133', 140f.', 163', 195'

šanû (syllabic) 'second': IGI 2: 216'; 'deputy of Ištar': NV 7'

šapāhu (syllabic) 'to sprinkle'

G-stem: ta-ša-pah: IGI 3: 6; NF 11'

šapāku (DUB(-ak)) 'to pour'

G-stem: tašappak(DUB(-ak)) IGI 1: 8’, 83’; IGI 2: 52’; ta-šap-pak-ma: NY 7a’ šapāru (syllabic) 'to send'

G-stem: lu-uš-pur: IGI 1: 102', 121'; ta-šap-pár: IGI 1: 160’

šaplānu (KI.TA-nu) ‘below’: IGI 1: 127’

šaptu (NUNDUM) 'lip': IGI 3: 90’

šaqālu (syllabic) 'to weigh'

G-stem: ta-ša-qàl: IGI 2: 151'

šaqu (NAG) 'to give to drink' $\rightarrow$ šatû

šarāku (syllabic) 'to present, give'

G-stem: iš-ru-ku-šú: IGI 2: 217’

šarāpu (syllabic) 'to burn'

G-stem: ta-šár-rap: IGI 2: 135'

šarmadu ('GÚR.UŠ) 'šarmadu-plant': IGI 2: 112'

šarru (LUGAL and 20) 'king': IGI 2: 217', 219’ 
šārtu (SÍG) ‘hair’: IGI 2: 17

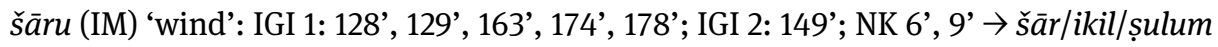

pāni

šār pāni (syllabic) 'head wind, resistance': IGI 1: 130'

šasû (syllabic and GÙ-si) 'to call, cry out'

G-stem: al-si-ki-na-ši: IGI 1: 114', 115’; i-šâ-as-si: IGI 1: 151'; išassi(GÙ-si): IGI 2: 12, 14

Gtn-stem: ši-ta-si-ia: IGI 2: 222'

šâšu (syllabic) 'of him': IGI 2: 107'

šâšunu (syllabic) 'those': IGI 2: 123’

šatti pāna (syllabic) 'spring': IGI 1: 183' $\rightarrow$ pānu

šatû (NAG) 'to drink'

G-stem: išatti(NAG): IGI 1: 26', 57'; IGI 3: 35', 72'

šâti $\rightarrow$ šuātu

šațāru (syllabic) 'write'

G-stem: áš-țùr: IGI 2: 221'

$\check{s} e^{\prime} u$ (ŠE) 'grain' $\rightarrow$ habbur še'i

šediš (6) 'six': IGI 1: 29'

šēlu (syllabic) 'sharp': IGI 2: 201'

šemû (syllabic) 'to hear'

G-stem: še-me-ma?: IGI 3: 85’; liš-ma-a: IGI 3: 85’

šer'ānu (SA(-a-nu)) 'sinew/tendon': IGI 1: 75'

šerhu (syllabic) 'furrow': IGI 1: 195'

šèrtu (syllabic) 'morning': NZb 11'

šêru (syllabic and UZU) 'flesh': IGI 1: 74'; IGI 2: 19, 22, 25, 26; IGI 3: 45', 75'

NT 2'

šêru (syllabic and Á.GÚ.ZI.GA) 'morning’: IGI 1: 38', 81'; IGI 3: 29’, 76’; NF 19’, 22’

šeššet $\rightarrow$ šediš

šibru (syllabic) 'broken': IGI 2: 67' $\rightarrow$ akalu

šihiltu (syllabic) 'filtered liquid': IGI 2: 154'

šikaru (KAŠ) 'beer’: IGI 1: 15’, 18’, 26’, 200’; IGI 2: 1, 56’, 181'; IGI 3: 35’, 72'; NK 7’; NR 7

šikar rūšti(KAŠ.SAG) 'premium beer': NQ 9'

šiknu (syllabic) '(blood) deposit (in the eye)': IGI 1: 61'

šilu (syllabic) 'perforation of the eye(lid)': IGI 3: 68', 70'

šimbizidû (ŠIM.BI.ZI.DA and šim-bi-zi-dam) 'šimbizidû-galena': IGI 1: 47’; IGI 3: 39'

-šina (syllabic) 'them, their, whom': IGI 1: 93', 101', 103', 120', 122', 126'-129', 177’; IGI 3:

68 '

šina (syllabic) 'two': IGI 1: 101', 126', 177'

šināti amēlūti (KÀŠ a-me-lu-ti) 'human urine' NF 34'

šinātu (KÀŠ) 'urine’: IGI 2: 189'

šinī̌su (2-šú) ‘twice, two-ply’: IGI 1: 185' $\rightarrow$ ištiššu šinišu šalāšišu

šīpātu peșâtu (SÍG BABBAR) ‘white wool’: IGI 1: 39', 97', 117’, 118', 143’, 154', 180', 181';

NU 13'; NZb 3' 
šīpātu sāmātu (SÍG SA $)$ 'red wool’: IGI 1: 109', 117’, 118', 143’, 154', 180', 181'; NF 44'; NZb 3'

šipru (syllabic and KIN) 'effect': IGI 2: 54'; 'skill': IGI 2: 219'

šiptu (syllabic and ÉN) 'incantation': IGI 1: 30’, 65’, 69', 70, 73’, 89', 94', 95', 97’, 106', 107', 109', 110', 115', 117', 119', 125', 132', 136', 144', 145', 147', 150', 154', 155', 157', 159', 163', 171', 176', 182', 186', 187', 191', 194'; IGI 3: 24', 85', 87'; NP 1'; NU 16'; NZb 6', 13'

šīpu (ŠIM.BI.KÙ.GI) ‘šīpu-paste’: IGI 1: 7’, 13’

šiqlu (GÍN) 'shekel’: IGI 1: 29’, 41', 64’; IGI 2: 55’, 92’, 140’, 141'; IGI 3: 49’, 50’; NK 13’, 14'; NS 6', 7'

šišitu (syllabic) 'membrane': IGI 2: 121', 124', 139'

šitta (syllabic and 2) 'two': IGI 1: 101', 120', 126'

šittu (syllabic) 'remnant, rest': IGI 2: 135'

šizbi enzi (GA UD $)_{5}$ 'goat's milk': IGI 1: 78'

šizbi musukkati (GA munusU.ZÚG) 'milk from a woman in maternity': IGI 1: 37’, 47’; IGI 2: 155', 205’: IGI 3: 39’;

šizbi musukkati ša zikara ūlidu (GA munuś.ZÚG šâ NITA Ù.TU) 'milk from a woman in maternity, who gave birth to a male': IGI 2: 148'? NR 5'

šizbu (GA) 'milk': IGI 1: 19', 35'; IGI 2: 65', 69', 192'; NQ 8'; NZ 10'

šu 'his, its, him' (pronominal suffix): IGI 1: 7', 8', 10'-15', 17', 18', 20'-26', 28', 29', 31'42', 44'-46', 48'-50', 56', 57', 59', 61', 63', 64', 68', 69', 71', 72', 77'-80', 84'-88', 93', 97', 105', 109', 114', 118', 144', 146', 148', 154', 166'-168', 174', 181', 184'-186', 197', 200', 201'; IGI 2: 1-7, 9, 12, 16-19, 22, 24, 25, 27, 52'-56', 58', 60', 61', 63', 66', 69', 76', 78', 89', 91, 97'-99', 101', 106', 108', 115'-119', 121', 123'-127', 132', 136', 137', 139', 142', 143', 144', 145', 158', 159', 169', 175', 181', 185', 190', 191', 194', 196', 199', 200', 202', 204', 207', 208', 213', 215', 216', 217'; IGI 3: 1-3, 6, 8, 9, 11, 30', 31', 35', 37', 39', 43', 49'-51', 53', 54', 57', 61', 67', 68', 70', 71', 75', 76', 78', 81', 83', 88', 90', 103'; NF 7', 12', 13', 16', 19', 20', 30', 33', 46'; NK 5'-7', 9', 15'; NP 7'; NQ 5', 8', 10', 11'; NR 2', 3’, 5’, 6', 10'; NS 1', 4’, 6’, 8'; NT 4'; NU 4'-7', 10', 12'; NV 1', 6', 7';

NW 4'; NZb 6', 7'; NY 4', 7a'; NZ 4', 8'; NZ 19'; NZa 8'-10'

šū (syllabic and BI) 'this same, that': IGI 1: 168'; IGI 2: 54'; IGI 3: 10, 49'; NS 6' šuātu (syllabic and BI) 'that, this': IGI 1: 166', 169'; IGI 2: 54', 219'; IGI 3: 77’, 84 ’ šubû (na4ŠUBA) 'šubû-stone’: IGI 3: 4

šubultu (syllabic) 'head-of-grain': IGI 1: 196' šugidimmakku (ŠU.GIDIM.MA) 'hand of a ghost': IGI 3: 2, 9, 10, 32', 43'; NV 7' šuharru $\rightarrow$ šaharru

šuhtu (SAHAR.URUDU) 'copper patina': IGI 2: 51', 62', 64', 71', 93’, 101'-104', 147', 154', 157’, 158'; IGI 3: 53’; NW 6' $\rightarrow$ šuhtu ša aškāpi/tangussi

šuhtu ša aškāpi (šu-uh-ta ša AŠGAB) 'patina from a leatherworker': IGI 1: 12' šuhtu ša tangussi (SAHAR.URUDU ša ŠEN.TUR) 'patina of a copper tangussu-kettle’:

IGI 1: 14', 16', 61'; IGI 2: 95'

šukkulu (syllabic) D-stem: 'to wipe, rub: tu-šak-kal: IGI 2: 3 
šumēlu (GÙB) ‘left (side)': IGI 1: 148’; IGI 2: 110’ $\rightarrow$ ìn šumēlišu, larsīni ša halli lahri ša šumèli ša māšš ūlidu

šumēnu ('na4ŠU.MÌN) 'šumēnu-mineral': IGI 1: 84'

šumma (DIŠ and šum 4 -ma) 'if': IGI 1: 10', 12', 14', 16', 21', 23', 26', 32', 34'-36', 40', 45', 57', 59', 61', 71', 77', 79', 87'-88', 184', 200', 201'; IGI 2: 1, 9, 12, 16, 17, 19, 22, 25, 27 , 53', 54', 56', 58', 60', 61', 63', 76', 115'-117', 121', 124', 126', 137', 139'-141', 147'-149', 159', 191', 196', 199', 200', 202', 204', 207', 209', 213', 215', 216'; IGI 3: 1, 9, 38'-40', 49', 51', 57', 66', 68', 70', 73'-75', 81'; NF 13', 15', 21', 26', 32', 33'; NK 6', 9'; NQ 5', 8', 10'; NR 3', 5', 6', 10'; NS 1', 6'; NT 4'; NV 1', 6’; NZ 19'; NZa 8'; 'when': IGI 1: 63'; NK 14'; NR 7'

šūmū (SUM ${ }^{\text {sar }}$ ' 'garlic': IGI 1: 15' $\rightarrow$ qilip šūmī

šunnu'ā $\rightarrow$ šanā'u

šunu 'their' (3 m. pl. gen.): IGI 1: 161', 180'; IGI 2: 105', 153', 156', 166'; IGI 3: 45'

šunû (giššE.NÚ.A) 'šunû-tree’: NF 18' $\rightarrow$ arti šunê, qēm šunê

šurru (syllabic) D-stem: 'to bow down, lean over': šur-ra-ta: IGI 1: 74'

šurrû (syllabic) 'beginning': IGI 1: 194'

šuršu (SUHUŠ) 'root' $\rightarrow$ šuruš ašāgi/ēri/gišimmari zikari/šammi rapādi/urâni

šuršummū (syllabic) 'sediments/dregs of beer: IGI 1: 15'; NQ 9'

šuršurru (syllabic) ‘šuršurru-fibre?’: IGI 1: 188'

šuruš ašāgi (SUHUŠ KIŠI ${ }_{16}$ ) 'root of ašāgu-acacia': NZa 9'

šuruš èri (SUHUŠ gišMA.NU) 'root of ēru-tree': NU 13'

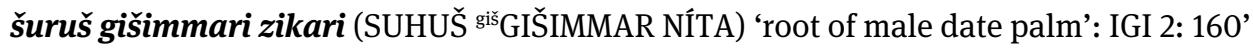
šuruš šammi rapādi (ŠUHUŠ Ú ra-pa-di) 'root of rapādu-disease-plant': IGI 1: 38' šuruš urâni (ŠUHUŠ 'HA) 'root of urânu-plant': IGI 1: 69'

šuššu (IGI.6.GÁL.LA) 'one-sixth': IGI 1: 41'

šūtu (IM.1) 'south wind': IGI 1: 115’

$\mathbf{T}$

tabālu (syllabic) 'take away, remove'

G-stem: tab-la: IGI 2: 13, 15

tābīla (syllabic) '(plant) in dry state’: IGI 1: 42’, 53’, 56’; IGI 2: 4, 6, 140’, 152'; NR 9'; NS

5'; 'eye dryness': IGI 1: 26'

tahāhu (syllabic) 'to drench' $\rightarrow$ rahāṣu

D-stem: tu-tah-ha-ah: IGI 1: 11'

tāhīzu (syllabic) 'teaching': IGI 2: 220'

takāpu (syllabic) 'to prick'

D-stem: tu-ták-kap: IGI 2: 105’, 166’

takkussu ('SAG.KUD) 'reed-straw': IGI 1: 7'; IGI 2: 115', 132'

tallu (syllabic) 'flask': IGI 1: 103'

taltallû ša gišimmari (tal-tal-lu-ú šá gišGIŠIMMAR) 'pollen of date palm’: IGI 1: 113’ 
tamāhu (syllabic) 'to grasp, seize’

N-stem: it-ta-mu-uh: NY 2'

tāmartu (syllabic) 'viewing': IGI 2: 222'

tâmtu (syllabic) ', sea, lake, ocean': IGI 1: 104' $\rightarrow$ imbu’ tâmti, rapšu

tangussu (ŠEN.TUR) ‘copper tangussu-kettle’: IGI 2: 86', 94’; NU 3’ $\rightarrow$ šuhtu ša tangussi

tarāṣu (LAL) 'to spread out (a cloth)'

G-stem: tatarraș(LAL-aș): IGI 3: 77’

tarbașu (TÙR) 'animal stall': IGI 1: 138'

tarmuš ('tar-muš, 'tar-muš) 'tarmuš-lupine': IGI 1: 46'; NU 4'

târu (GUR) 'to return, repeat'

G-stem: itūr(GUR): IGI 1: 80'; tatârma(GUR-ma): NZ 23'

D-stem: tuttanār (GUR.GUR): IGI 2: 119'

tê šipti (TU6.ÉN) 'incantation spell': IGI 1: 67', 70', 95', 107', 123', 130', 134', 145', 152',

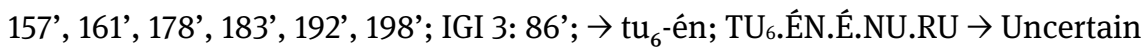

Readings and Words

tebû (syllabic and ZI) 'to rise, throb'

G-stem: it-ba-ki-na-ši: IGI 1: 115'; tebìma(ZI-ma): NZa 8'

temēru (syllabic) 'to bury'

G-stem: te-te-mer: IGI 2: 28, 59', (te-tem-mer) 103’

tēqìtu (syllabic) 'ointment': IGI 2: 26; IGI 3: 32'

tēqìtu šalimtu (syllabic) 'healing ointment': IGI 3: 96'

tibnu (IN.NU) 'straw': IGI 1: 114'

tību (ZI) 'rising (of wind)': IGI 1: 129'

tinūru (NININDU) 'tannour-oven': IGI 2: 65', 98'; IGI 3: 71'; NF 35’; NR 8'

tiše, tišît (9) 'nine': IGI 3: 44', 98', 101'

tittu (syllabic and gišPĚS̆) 'fig': IGI 1: 114'; NF 18', 21' $\rightarrow$ arti titti

tìyatu (syllabic) 'tìyatu-plant' $\rightarrow$ hīl tīyati

tû $\left(\mathrm{TU}_{6}\right)$ 'incantation': IGI 1: 75'

tubkinnu (syllabic) 'hole, refuse heap': IGI 1: 189'

talupadu (syllabic) 'talupadu-plant' $\rightarrow$ zèr talupadi

tupšarrūtu (syllabic) 'scribal art': IGI 2: 218'

tuškû ("na4)tu-uš-ka-a) 'tuškû-mineral': IGI 1: 11’, 82'; IGI 3: 104'

T

țābātu (A.GEŠTIN.NA) 'vinegar': IGI 1: 81'; IGI 2: 145’; (t. bašlāti) NZ 25’ $\rightarrow$ karpat țābāti țābtu (MUN) ‘salt’: IGI 2: 17, 71', 112’; NR 3’ $\rightarrow$ baltu ša țābti, hașab țābti țābu (DU $\mathrm{DU}_{10}$ 'sweet' $\rightarrow$ qanû țābu

țābu (syllabic) 'good, friendly': NF 19'

tâbu (syllabic) 'to be good'

G-stem: i-țàb: IGI 1: 183’ 
țamû (NU.NU) 'to spin'

G-stem: tațammi(NU.NU): IGI 1: 39', 97', 109', 117’, 143’, 154', 180', 185’; NZ 12'

tebētu $\rightarrow$ Month Names

tehû (TE) 'to be(come) near to, approach'

G-stem: țehe(TE-e): NF 17’; ițehhe(TE): NF 20', 23’; iț-hi-ka: NZb 9’, 10’

D-stem: tetehhima(TE-ma) 'you introduce': IGI 2: 98'

tênu (ÀRA) 'to grind up'

G-stem: tetên(ÀRA-en): IGI 2: 190’

tepû (syllabic and TAB) 'to apply (drugs), add (ingredients)'

G-stem: ta-țe-ep-pi: IGI 1: 53’; te-țe-ep-pí: IGI 2: 67’; te-țep-pi: NS 5’

terû (syllabic) 'to ooze'

G-stem: iț-ri: NR 3', 5', 10'; ți-ri: NR 6'

țipu (syllabic) 'drop(s)': IGI 2: 9, 10, 11, 57’, 67’; NK 8'

tuppu (syllabic and DUB) 'clay tablet': IGI 1: 201'; IGI 2: 216', 221'

țurru (DUR) 'a cord': IGI 1: 39', 97’, 109', 118', 185’; NF 44’; NU 13’; NZ 12'

țūru (šimHAB) 'țūru-plant': IGI 2: 16; IGI 3: 52'

\section{U}

u (syllabic) 'and': IGI 1: 14', 21', 36', 39', 46', 54', 70', 84', 94', 95', 106', 117', 123', 138', 152', 154', 159', 161', 178', 185', 186', 190', 192', 193', 198'; IGI 2: 9, 17, 22, 25, 58', 61', 64', 77', 93', 98', 138', 214', 217', 221'; IGI 3: 8, 34', 50', 67', 83', 90', 100', 103'; NF 13', 14', 18', 31', 33', 34'; NK 10', 15'; NQ 5', 10'; NS 1', 8'; NT 2', 3’; NV 6'; NW 6' $\bar{u}$ (syllabic) 'or': IGI 2: 5 (gloss)

ubān hašê (ŠU.SI ha-še-e) 'lobe of the lung': IGI 3: 87'

ubāni-ași (syllabic) 'ubāni-ași plant': IGI 2: 153'

ubānu (ŠU.SI) 'finger’: IGI 1: 84’; IGI 2: 73'; IGI 3: 95'

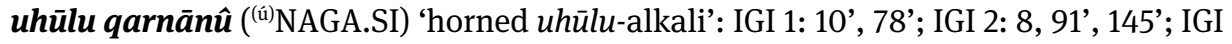
3: 52', 53', 55', 94', 104'; NK 2'; NR 6'; NU 14'; NV 1' $\rightarrow$ mê uhūli qarnānî

ul (syllabic and NU) 'no, not': IGI 1: 67’, 94’, 106', 115’, 121', 152'; IGI 2: 149’; IGI 3: 30’,

54'; NF 30'

ummânu (UM.ME.A) 'expert, sage’: IGI 3: 96’ $\rightarrow$ qātu

ummātu (syllabic and É.MEŠ, AMA.MEŠ) ‘summer’: IGI 1: 52’, 63’; (NR 8’)

ummu I (AMA) 'mother': IGI 1: 177'

ummu II (KÚM) 'ummu-fever (lit. heat, summer)': IGI 1: 23', 105'; IGI 2: 117’; NF 33’

ūmu (UD-(me/mi)) ‘day’: IGI 1: 23', 24'; IGI 2: 7, 28, 59', 95', 103', 117’, 118'; IGI 3: 44', 73', 74' $\rightarrow$ kala ūme

uppi siparri (MUD ZABAR) 'a bronze tube': IGI 1: 86’-88'

upputtu (syllabic) 'upputtu-insect' $\rightarrow$ zê upputte

uqnâtu ('ZA.GìN.NA) 'uqnâtu-blue-plant': IGI 1: 69'

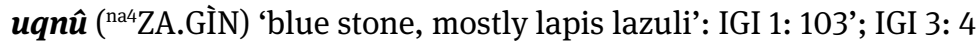




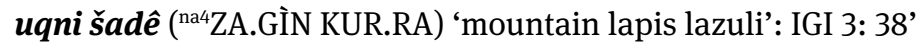

urânu ('HA) 'urânu-plant': NV 2' $\rightarrow$ šuruš urâni

urnû ('úr-né-e) 'urnû-plant': IGI 1: 88'; IGI 2: 87'

urru (syllabic) 'day': IGI 1: 36'

urruru (syllabic) 'to parch'

D-stem: tur-âr: IGI 1: 9', 16', 24', 27', 37'; IGI 2: 67', 70', 75', 102', 118', 146'

ūru (ÙR) 'roof'; IGI 3: 28'; NF 27'

ušû ('ESI) 'ušû-plant': IGI 3: 53'

utțatu (ŠE and ŠE.BAR / KU.KU) 'grain (also as a measure)': IGI 1: 44', 78'; IGI 2: 139',

141'; IGI 3: 27’; NT 9’ $\rightarrow$ kalìt lalî lakî ša šamma lā našku uțtata lā ṣabitu

uznu (GEŠTU) 'ear, understanding': IGI 2: 217'; NK 15'

uzun lalî ('UR.TÁL.TÁL) 'young goat's-ear plant': IGI 2: 145’

\section{W}

wabālu $\rightarrow$ abālu

walādu $\rightarrow$ alādu

warā $d u \rightarrow$ arā $d u$

wardatu $\rightarrow$ ardatu

wașû $\rightarrow$ așû

$\mathbf{Y}$

$y a \rightarrow \bar{\imath}$

yā'uttun (syllabic) 'mine': IGI 1: 94', 106'

\section{Z}

zagindurû ( ${ }^{\text {na4ZZA.GÌN.DURU }}$ ) 'zagindurû-lapis lazuli': IGI 1: 122'

zakû (syllabic) 'to become clear'

D-stem: ú-za-ku-ú: IGI 2: 119'

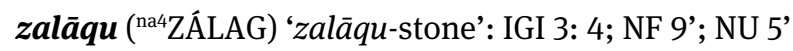

zanānu (syllabic) 'to rain'

G-stem: zu-un-na-ni: IGI 1: 189’

zappi šahî (zap-pí ŠAH) 'pig’s bristle'; NZ 20'

$z \hat{a} q u$ (syllabic) 'to blow'

G-stem: i-zi-qam-ma: IGI 1: 163’

zarû (syllabic and MAR(-ru)) 'to scatter, spatter, sprinkle'

G-stem: tazarru(MAR(-ru)) IGI 1: 26’, 42'; IGI 2: 4, 6, 140'; NZ 24'; ta-zar-ri: IGI 1: 50’ 
$\boldsymbol{z} \hat{\boldsymbol{e}}$ șurāri (ŠE $\mathrm{E}_{10}$ EME.ŠID) 'lizard's excrement': IGI 1: 43', 78'

zê upputte (ŠE $\mathrm{E}_{10}$ up-pu-te) 'excrement of upputte-insect': IGI 2: 109'

zēer ašli (NUMUN ${ }^{(\mathfrak{u})} a$ ášli) 'seed of rush': IGI 3: 5, 7; NY 4'

zēr ašli zikari (NUMUN áš-li NITA) 'seed of male rush': IGI 3: 5

zēr bīni (NUMUN ŠINIG) 'seed of tamarisk': IGI 2: 16; IGI 3: 5, 7; NF 10', 45'; NT 11'

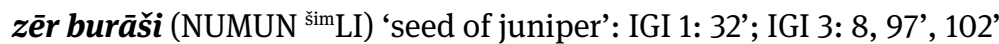

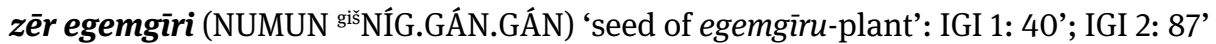

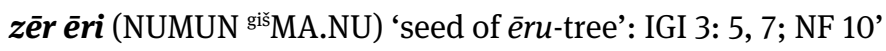

zēr GI.ZÚ.LUM.MA (NUMUN GI.ZÚ.LUM.MA) 'seeds of GI.ZÚ.LUM.MA-plant’: IGI 2: 20, 196', 206'

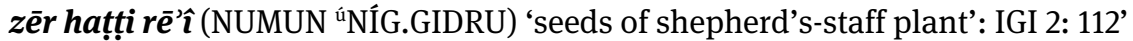

zēer lišān kalbi (NUMUN 'EME.UR.GI 7 ) 'seeds of dog's-tongue plant': IGI 2: 112', 122'

zēer maštakal (NUMUN úIN.ÚŠ) 'seeds of maštakal-plant': IGI 1: 24'; IGI 2: 118'

zēr qudri (NUMUN úKI. 'IŠKUR) 'seeds of qudru-plant': IGI 2: 5

zēer šammi daqqiqi (NUMUN Ú da-aq-qí-qí) 'tiny' plant seeds': IGI 2: 109'?

zēr talupadi (NUMUN úta-lu-pa-di) 'seeds of talupadu-plant': IGI 2: 109'

$z \bar{e} r u$ (NUMUN) 'seed(s)': $\rightarrow$ zēr ...

zību (syllabic and GAMUN.GE6) 'black cumin': IGI 1: 82'; IGI 3: 47’, 52', 107'

zidubdubb̂̂ (Zİ.DUB.DUB.BU) 'a heap of flour': NF 29'

zikaru (NÍTA, NITA) 'man, male': NF 27'; NR 5' $\rightarrow$ arti pillî zikari, muhhu ša summati

zikari, nikiptu zikar u sinniš, pillû zikaru, šizbi musukkati ša zikara ūlidu, šuruš gišimmari zikari, zēr ašli zikari

$z$ û (ŠE $\left.\mathrm{E}_{10}\right)$ 'excrement' $\rightarrow$ zê șurāri

zumru (SU) 'body': NF 33'

$z$ ūtu (IR) ‘sweat'; NF 34' $\rightarrow$ rašû 


\section{Sumerian Words}

a 'water': IGI 1: 150'

-a 'in': IGI 1: 164', 165', 174'; 'with' IGI 1: 173’

a-ga 'back': IGI 1: 73', 155'

a-lal-la 'shaduf': IGI 1: 150'

abzu 'underground water': IGI 1: 171'

ad-da 'father': NF 40'

an 'sky': IGI 1: 164'

bàn-da 'small/tiny': IGI 1: 155'

bar 'open': IGI 1: 89', 90', 98', 99', 110', 111', 119', 125', 132', 133’

bar-bar 'staring': IGI 1: 89', 98', 110', 119', 125', 132'

bar-ra 'opened': IGI 1: 89', 98', 110', 119', 125', 133'

-bi 'its, this, his': IGI 1: 157', 166'-169', 173', 174', 182'; NF 36'-39', 42'

da 'weak': IGI 1: 90', 99', 111'

di-ku 'judge': NF 42'

dumu 'son': NF 40'

dumu-sag 'first-born son': NF 40'

è 'to leave': IGI 1: 174'

én 'incantation': NF 36'

ér 'tears': IGI 1: 150'

ér šěš-šéš 'to pour out (tears), weep’: IGI 1: 168'

gar 'to place/cause': IGI 1: 164', 165'

gaz-gá 'crushed': IGI 1: 170'

geštu 'ear': IGI 1: 73'

gi 'reed': IGI 1: 155'

gi-na 'to be firm/true, to be in order': IGI 1: 73', 133', 155', 157'

gig 'sick, bitter': IGI 1: 168', 175', 179'

gig-ga 'sore, lesion, wound': IGI 1: 163'-165'

$\operatorname{gin}_{7}$ 'like': IGI 1: 150'

gù dé 'to say, talk, speak': NF 40'

gub 'to stay, stand, be present': IGI 1: 65', 150'

hé-pà 'may adjure'; NF 36'-39'

hul 'evil, harmed': IGI 1: 90', 99', 111', 119'

hul-hul 'malevolent': 119'

huš 'reddish': IGI 1: 89', 98', 110', 157'

huš-huš 'crimson: IGI 1: 89', 98', 110', 132'

igi (and syllabic i-gi) 'eye’: IGI 1: 73', 89', 90', 96', 98'-99', 108', 110', 111', 116', 119',

124', 125', 131'-133', 135', 149', 155', 158', 162-167', 169', 172-175', 179', 182'; NF 36'-

39' (in rubrics, it is uncertain if not realized in Akkadian)

igi bar 'to see' NF 41

igi sì 'to see, look': IGI 1: 169' 
im 'wind': IGI 1: 163', 174'

im ri 'to blow wind' IGI 1: 163', 164'

im sù-sù 'to blow': IGI 1: 174

kešda 'to bind': IGI 1: 172'

kimin 'ditto': $\rightarrow$ ašar šanîmma

kù-ga 'clean': IGI 1: 173'

kum 'crushed': IGI 1: 170'

kún 'shining': IGI 1: 73': 155'

lá 'winnowed': IGI 1: 182'

lal 'diminished': IGI 1: 182'

lú 'a man': IGI 1: 163’, 164', 166', 169', 172'-174'

lú-u ur $_{18}-\mathrm{lu}:$ IGI 1: 168'

lúa-zu 'asû-physician' NF 42' $\rightarrow$ asû

lù 'troubled': IGI 1: 166'

min 'ditto': IGI 1: 182'

-mu 'my': NF 40', 41'

ná 'to be drowsy': IGI 1: 90', 99', 111'

nam-tar 'to make decision': NF 41'

-ni 'his': IGI 1: 169', 173'

ní-te-a-ni-šè 'by himself': IGI 1: 168'

níg-gi-na-bi 'its firm thing, in truth': IGI 1: 157'

nu 'not': IGI 1: 132'

numun 'seed': IGI 1: 65'

pà 'adjure' $\rightarrow$ hé-pà

pa-la 'robe': IGI 1: 157'

ri $\rightarrow$ im ri

sag-hul-ha-za 'supporter-of-evil': NF 36'-39', 43’ $\rightarrow$ mukīl rēš lemutti

sì $\rightarrow$ igi sì

sì $\rightarrow \mathrm{tu}_{6}-\mathrm{tu}_{6}$ sì

sù-da 'distant': IGI 1: 164'

sù-sù 'to inflate, blow into': IGI 1: 174'

sùh 'blurred': IGI 1: 125'

sùh-sùh 'to blurr a lot': IGI 1: 125', 167'

sùh-sùh 'very blurred': IGI 1: 125'

še 'grain': IGI 1: 182'

šéš-šéš $\rightarrow$ ér šéš-šéš

šu 'hand': IGI 1: 173' $\rightarrow$ tag, ti

šu tag 'to touch': IGI 1: 173'

šu ti 'to take: IGi 1: 170'

ta 'from'; IGI 1: 171', 174'

tag $\rightarrow$ šu tag

ti $\rightarrow$ šu ti 
ti-la 'healthy': IGI 1: 73', 155'

tu-ra 'illness; the sick': IGI 1: 169'

tu 6 -én 'incantion spell': IGI 1: 147', 155', 157'; NF 42'; NY 6'

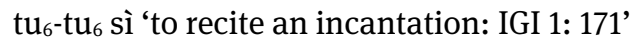

ú-hu-ur-sag-gi-na 'the real mountain plant': IGI 1: 73'

ur-sag 'hero': IGI 1: 155'; NF 36'-39'

zu 'your': IGI 1: 155' 


\section{Logograms with Cuneiform Signs}

\section{Notes:}

- Cuneiform signs follow Neo-Assyrian forms as reflected in Nineveh.

- Logograms are also designated by the name and the number appearing in Borger 2010, Mesopotamisches Zeichenlexikon - in round brackets: e.g. (ŠU, no. 567). In case the logogram is missing in Borger 2010, it is marked with 'not in MZ.'

\section{A}

A.GA.ÁŠ.GA (not in MZ) $\rightarrow$ agašgû 'novice (physician)'

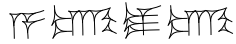

A.GAR.GAR (A, no. 839) $\rightarrow$ piqannu 'droppings (of gazelle)'

TF PO

A.GAR $_{5}(\mathrm{~A}$, no. 839) $\rightarrow$ abāru 'lead'

FF

A.GEŠTIN.NA (A, no. 839) țābātu 'vinegar' $\rightarrow$ DUG A.GEŠTIN.NA

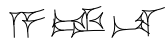

Á.GÚ.ZI.GA (Á, no. 560) $\rightarrow$ šēru 'morning'

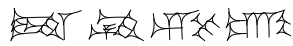

A.GÚB.BA.A (A, no. 839) $\rightarrow$ agubbû 'holy water vessel'

FF N W W

A.HA.TAR.DU $=$ GİRIMA (A, no. 839, Kap. II $) \rightarrow{ }^{\mathrm{d}}$ Nin-girima

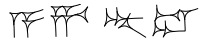

$\mathrm{A}(. \mathrm{MEŠ})(\mathrm{A}$, no. 839) $\rightarrow$ mû 'water'

FF( KLAB)

AMA.MEŠ (not in MZ) $\rightarrow$ ummātu 'summer'

W KAH

A.ZU-ti (not in MZ) $\rightarrow$ asûtu 'medical skill'

Fि UT UF

AN.BAR (AN, no. 10) $\rightarrow$ parzillu 'iron'

YFH

AN.ŠÁR ${ }^{k i}(A N$, no. 10) $\rightarrow$ Aššur 'Assyria'

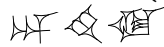

$\mathrm{AN}(-e)(\mathrm{AN}$, no. 10) $\rightarrow$ šamû 'sky/heaven'

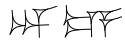

ANŠE (ANŠE, no. 353) $\rightarrow$ imēru 'donkey'

AA

AN.ZAH.GE 6 (AN, no. 10) $\rightarrow$ kutpû 'black frit (bead)'

H कर 
ÀRA (HAR, no. 644) $\rightarrow$ tênu 'to grind up'

多等

$\mathrm{ARGAB}^{\text {mušen }}$ (NÍG, no. 859) arkabu 'bat' $\rightarrow \mathrm{U}_{5} . \mathrm{ARGAB}^{\text {mušen }}$

एW WAT

AŠ (A ̌s, no. 1) $\rightarrow$ ina 'in, on'

$\succ$

\section{B}

BABBAR (UD, no. 596) $\rightarrow$ peșû 'white'; pūșu 'white spot' \&े

BABBAR.HI ${ }^{\text {sar }}$ (UD, no. 596) $\rightarrow$ papparhû 'papparhû-purslane'

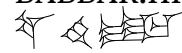

BAD (BAD, no. 113) $\rightarrow$ petû 'to open'<smiles>C1=CCCC1</smiles>

BAL (BAL, no. 5) $\rightarrow$ naqû 'to pour out (tears, water)'

nabalkutu 'to cross over, change sides'

BAL.GI ${ }^{\mathrm{ku} 6}(\mathrm{BAL}$, no. 5) $\rightarrow$ raqqu 'turtle'

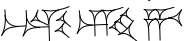

BAR (BAR, no. 121) $\rightarrow$ ahû 'stranger, non-canonical (material)'; mišlu 'half'

LF qilpu 'skin of plant, fruit, peel'

$\mathrm{BI}(\mathrm{BI}, \mathrm{no} .358) \rightarrow$ šù 'this same, that'; šuātu 'that, this'

$<4$

BIL (NE, no. 313) $\rightarrow$ qalû 'to roast'

ar

BÍL (BÍL, no. 312) $\rightarrow$ qalû(BIL)

हैA

BIL.ZA.ZA (NE, no. 313) $\rightarrow$ mușa"irānu 'muṣa"irānu-frog'

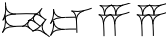

BÚN (BÚN, no. 54) $\rightarrow$ napāhu 'to blow; to light up, ignite' 这然

\section{D}

DAB (DIB, no. 813) $\rightarrow$ șabātu 'to seize, grasp, afflict'

kullu 'to hold, contain, keep, retain'

DADAG (UD, no. 596) $\rightarrow$ ebbu 'pure'

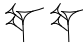

${ }^{\mathrm{d}} \mathrm{AG}(\mathrm{AG}$, no. 127) $\rightarrow$ Nabû 'god Nabû'

UF十 
DAGAL (AMA, no. 392) $\rightarrow$ rapšu 'wide, great'

WA

DIDA (BI, no. 358) $\rightarrow$ billatu 'beer mash'

犁

DÍLIM A.BÁR (LIŠ, no. 591) $\rightarrow$ itqūr abāri 'lead spoon(salve)'

Y F F

DIRI (DIR, no. 207) $\rightarrow$ malû 'to be(come) full of'

tim

DIŠ (DIŠ, no. 748) $\rightarrow$ ana 'to, for, on, onto (also in compound prep.)'; šumma 'if'

Y

${ }^{\mathrm{d} N i n-g i r i m a}(\mathrm{NIN}$, no. 887) $\rightarrow$ Ningirimma 'mistress of incantations'

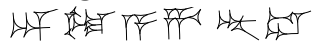

dŠE.TIR (ŠE, no. 579) $\rightarrow$ ašnan 'grain'

YF सूस्था

DU (DU, no. 350) $\rightarrow$ alāku 'to go (+ ventive to come)'

àliku 'moving, loose tissue in the eyes'

DÙ.A.BI (GAG, no. 379) $\rightarrow$ kalû 'everything, the whole of'

G度 4

DÙ.DÙ.BI (GAG, no. 379) $\rightarrow$ dudubi 'its ritual, medical application'

存席

DÙ (GAG, no. 379) $\rightarrow$ epēšu 'to do, make’

4

$\mathrm{DU}_{10}(\mathrm{HI}$, no. 631) $\rightarrow$ țābu 'sweet'

$\Delta$

$\mathrm{DU}_{8}(\mathrm{GAB}$, no. 298) $\rightarrow$ pațāru 'to loosen'

这

DUB (DUB, no. 242) $\rightarrow$ šapāku 'to pour'

sm

DUG (DUG, no. 499) $\rightarrow$ karpatu 'a vessel'

部众

DUG A.GEŠTIN.NA (DUG, no. 499) $\rightarrow$ karpat țābāti 'vinegar vessel'

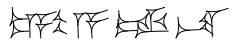

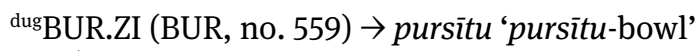

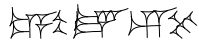

DUG $_{4 . G A}$ (KA, no. 24) $\rightarrow$ qabû 'to say, speak'

斯度

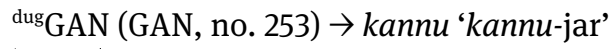

cris

DUGUD (DUGUD, no. 704) $\rightarrow$ kabātu 'to become heavy'

4

DUL (DUL, no. 720) $\rightarrow$ katāmu 'to cover objects, close the eye'

樯 
DUMU.MUNUS (TUR, no. 255) $\rightarrow$ mārtu 'daughter'

\section{年}

DUR (DUR, no. 178) $\rightarrow$ țurru 'a cord'

度

'UTU.È (UD, no. 596) $\rightarrow$ șìt šamši ‘sunrise’

4 क्षे

\section{E}

É (É, no. 495) $\rightarrow$ bitu 'house'

Exiा

È (È, no. 596) $\rightarrow$ așû 'to go, come out'; šakāku 'to twist, thread (on a string)'

姲牙

É.GAL (É, no. 495) $\rightarrow$ ēkallu 'palace'

प्राm

É.GAR $(\hat{E}$, no. 495) $\rightarrow$ igāru 'wall'

Tiा

É.MEŠ (É, no. 495) $\rightarrow$ ummātu 'summer'

TIIIS

É.NU.RU (É, no. 495) $\rightarrow$ TU6.ÉN.É.NU.RU

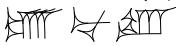

EGIR (EGIR, no. 356) $\rightarrow$ arki 'after(wards)'

供

ÉLLAG (BIR, no. 643) $\rightarrow$ kalitu 'kidney'

然

EME.DIR/ŠID (EME, no. 61) $\rightarrow$ șurāru 'lizard'

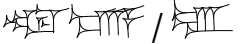

ÉN (ÉN, no. 870) $\rightarrow$ šiptu 'incantation'

还F

EN (EN, no. 164) $\rightarrow$ adi 'until'; bèltu 'mistress'

HU

EN.TE.NA (EN, no. 164) $\rightarrow$ kūṣu 'winter'

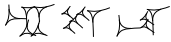

ÉR (A, no. 839) $\rightarrow$ dimtu 'tear(s)'

F⿸广 低

EREN (EREN, no. 818) $\rightarrow$ erēnu 'cedar tree'

$\Rightarrow$ W

ESIR (A, no. 839) $\rightarrow$ ițtû 'liquid bitumen’

TF 


\section{G}

GA (GA, no. 491) $\rightarrow$ šizbu 'milk'

牙部

GAB.LÀL (GAB, no. 298) $\rightarrow$ iškūru 'wax'

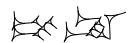

GAG.TI.TI (GAG.TI, no. 379) $\rightarrow$ sikkat șēli ‘(lamb) chop

प्राA

GAMUN.GE $_{6}$ (DIN, no. 119) $\rightarrow$ zībû 'black cumin'

雨

GAR (NÍG, no. 859) $\rightarrow$ šakānu 'to put, place, present'

एक

GAZ (GAZ, no. 340) $\rightarrow$ hašālu 'to crush'

应

$\mathrm{GAZI}^{\mathrm{sar}}\left(\mathrm{SILA}_{4}\right.$, no. 408) $\rightarrow$ kasû 'kasû-plant'

屎

$\mathrm{GE}_{6}(\mathrm{MI}$, no. 681) $\rightarrow$ mūšu 'night'; șalmu 'dark, black'

t

GEŠTIN.NA (GEŠTIN, no. 212) $\rightarrow$ karānu 'wine'

प⿺辶冋子

GEŠTU (PI, no. 598) $\rightarrow$ uznu 'ear, understanding'

很

GI (GI, no. 141) $\rightarrow$ qanû 'reed'

所政

GIDIM (GIDIM, no. 830) $\rightarrow$ ețemmu 'ghost'

II)

GI.ZÚ.LUM.MA (GI, no. 141) $\rightarrow$ Uncertain Readings and Words

舟度证在

GÍD.DA (BU, no. 580) $\rightarrow$ arku 'long (of sheep's bones)'

स्थ 很

GIG (GIG, no. 705) $\rightarrow$ marāṣu 'to be sick'; kibtu 'wheat'

解

GIG.GA (GIG, no. 705) $\rightarrow$ marșu 'sick'

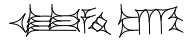

GIG.GIR (GIR, no. 558) $\rightarrow$ kurāru 'kurāru-pustle’

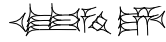

GIN (DU, no. 350) $\rightarrow$ kânu 'to establish, deposit, to set up'

प्र

GÍN (GÍN, no. 836) $\rightarrow$ šiqlu 'shekel'

mer

$\mathrm{GIN}_{7}(\mathrm{GIM}$, no. 686) $\rightarrow$ kìma 'like, as'

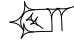


GÍR (GÍR, no. 6) $\rightarrow$ patru 'knife' $\checkmark$ QTा

GÌR.PAD.DU/DA (GÍR, no. 6) $\rightarrow$ eșemtu 'bone' 佂

GÍR.ŠU.I (not in MZ) naglabu 'barber's knife, scalpel'

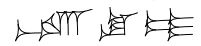

GÌRIMA $\rightarrow$ A.HA.TAR.DU

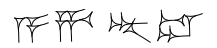

GISSU (GIŠ, no. 469) $\rightarrow$ șillu 'shadow/film (on/in the eye)'

许

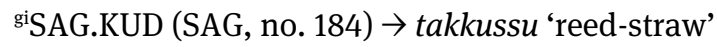

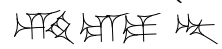

giš DÁLA (IGI, no. 724) $\rightarrow$ șillû 'needle'

प्र याद

giš́́RIN (GIŠ, no. 469) $\rightarrow$ gišrinnu 'scale'

प्र 4 if

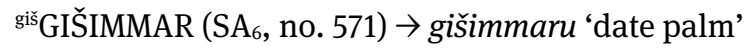

प्र

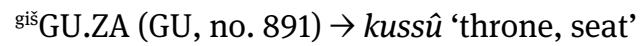

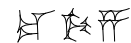

giš GÚR.GÚR (GAM, no. 576) $\rightarrow$ kukru 'kukru-aromatic'

可以出

gišHAB (LAGAB, no. 755) $\rightarrow$ hūratu'dyeing sumac'

tro

gišHAŠHUR (HAŠHUR, no. 270) $\rightarrow$ hašhūru 'apple'

许透

gišIG (IG, no. 136) $\rightarrow$ daltu 'door'

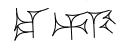

giš MA.NU (MA, no. 552) $\rightarrow$ ēru 'ēru-tree'

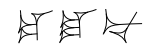

giš MAŠ.HUŠ (MAŠ, no. 120) $\rightarrow$ kalbānu 'kalbānu-plant (lit. dog-like plant)'

प्र Y Q

gišMI.PÀR/PAR 4 (MI, no. 681) $\rightarrow$ lipāru 'lipāru-tree'

प्र यदि /

gišNÁ (GIŠ, no. 469) $\rightarrow$ eršu 'bed'

पर

gišNAM.TAR (NAM, no. 134) $\rightarrow$ pillû 'male pillû-plant'

प्र

gišNÍG.GÁN.GÁN (NÍG, no. 859) $\rightarrow$ egemgīru 'egemgīru-plant'

प्रे

gišsU.ÚR.MA (NU, no. 112) $\rightarrow$ nurmû 'pomegranate'

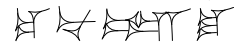


gišPĚŠ (MA, no. 552) $\rightarrow$ tittu 'fig'

谋草

gišŠE.NÚ.A (ŠE, no. 579) $\rightarrow$ šunû 'šunû-tree'

प्र

giššINIG (ŠINIG, no. 162) $\rightarrow$ bīnu 'tamarisk'

和产

${ }_{\text {giš KIŠI }}{ }_{16}(\mathrm{U}$, no. 490) $\rightarrow$ ašāgu 'ašāgu-acacia'

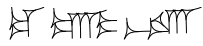

GÚ (GÚ, no. 176) $\rightarrow$ kišā du 'neck'

政

GÚ.GAL (GÚ, no. 176) $\rightarrow$ hallūru 'chickpea'

压

GÚ.TUR (GÚ, no. 176) $\rightarrow$ kakkû 'lentil'

两

$\mathrm{GU}_{4}\left(\mathrm{GU}_{4}\right.$, no. 472) $\rightarrow$ alpu 'ox'

原

$\mathrm{GU}_{7}\left(\mathrm{GU}_{7}\right.$, no. 65) $\rightarrow$ akālu 'to eat, consume, hurt, cause pain'

से

GUB (DU, no. 350) $\rightarrow$ izuzzu 'to stand, be present'

प्र

GÙB (KAB, no. 148) $\rightarrow$ šumēlu 'left (side)'

trá

GUR (GUR, no. 180) $\rightarrow$ târu 'to return, repeat'

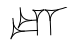

GURUN (GURUN, no. 503) $\rightarrow$ inbu 'fruit'

虹公

GURUŠ (KAL, no. 496) $\rightarrow$ eṭlu 'young man, lad'

êtr

\section{H}

HÂD.DU (UD, no. 596) $\rightarrow$ abālu 'to dry'

年 प्रत

HE.HE (HI, no. 631) $\rightarrow$ balālu 'to mix'

\&

HÉNBUR (ŠE.GAG, no. 579) $\rightarrow$ habburru 'sprout'

乐席

HUL (HUL, no. 733) $\rightarrow$ lemnu 'evil'

या山्य 
İ(.GIŠ), İ+GIŠ (NI, nos. 380, 436) $\rightarrow$ šamnu ‘sesame oil’ (t),

Borger 2010: 325 transliterates İ.ŠÉŠ mistakenly for İ.ŠEŠ

İ.GIŠ.BUR (BUR, no. 559) $\rightarrow$ šaman pūri 'pūru-oil'

WU

İ.KUR.RA (NI, no. 380) $\rightarrow$ napțu 'naphtha'

出较

İ.NUN(.NA) (NI, no. 380) $\rightarrow$ himētu 'ghee'

(1)

İ.UDU (NI, no. 380) $\rightarrow$ lipû 'fat'

蕞

IGI (IGI, no. 724) $\rightarrow$ inu 'eye'; națālu 'to see'; pānu 'front, face'; mahru 'in front' या amāru 'to see'

IGI.4.GẤL.LA (IGI, no. 724) $\rightarrow$ rebiat 'one-fourth'

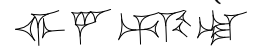

IGI.6.GÂL.LA (IGI, no. 724) $\rightarrow$ šuššu 'one-sixth’

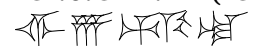

IGI.DU $_{8}(\mathrm{IGI}$, no. 724) $\rightarrow$ amāru 'to see'

याए

IGI.KÁR (IGI, no. 724) $\rightarrow$ barû 'to collate (a tablet); to check (a remedy)'

या

IGI.SIG.SIG7 (IGI, no. 724) $\rightarrow$ amurriqānu 'jaundice'

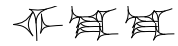

ÍL (ÍL, no. 493) $\rightarrow$ našû 'to carry, lift, + ventive to bring, carry along, pick up'

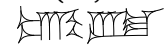

ILLU (A, no. 839) $\rightarrow$ hìlu 'resin'

庐校

IM (IM, no. 641) $\rightarrow$ šāru 'wind'

Sर्या

IM.1 (IM, no. 641) $\rightarrow$ šūtu 'south wind'

S这个

IM.2 (IM, no. 641) $\rightarrow$ iltānu 'north wind'

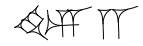

IM.3 (IM, no. 641) $\rightarrow$ šadû 'east wind'

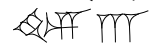

IM.4 (IM, no. 641) $\rightarrow$ amurru 'west wind'

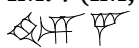


IM.GÚ.EN.NA (IM, no. 641) $\rightarrow$ qadūtu šikāni 'river silt'

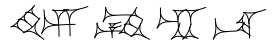

IM.SAHAR.BABBAR.KUR.RA (IM, no. 641) $\rightarrow$ alluharu 'alluharu-mineral'

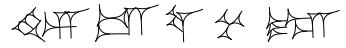

IM.SAHAR.NA4.KUR.RA (IM, no. 641) $\rightarrow$ gabû 'gabû-alum'

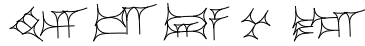

imKAL (KAL, no. 496) $\rightarrow$ kalû 'kalû-paste'

Q

imKAL.GUG (KAL, no. 496) $\rightarrow$ kalgukku 'kalgukku-red paste'

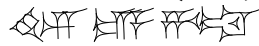

IN.NU (IN, no. 261) $\rightarrow$ tibnu 'straw'

\&

IN.NU.UŠ (IN, no. 261) $\rightarrow$ maštakal 'maštakal-plant'

\&

IR (IR, no. 437) $\rightarrow z \bar{u} t u$ 'sweat'

TIR

ITI (ITI, no. 20) $\rightarrow$ arhu 'month'

(T)

itiBÁRA (ITI, no. 20) $\rightarrow$ Nisannu 'month Nisannu'

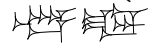

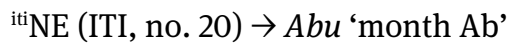

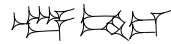

${ }^{\text {iti }} \mathrm{AB}$ (ITI, no. 20) $\rightarrow$ Tebètu 'month Tebet'

制

ITI.1.KÁM $\rightarrow$ ITI.1.KAM in Uncertain Readings and Words

Y

IZI (IZI, no. 313) $\rightarrow$ išātu 'fire’

tor

K

KÁ (KÁ, no. 222) $\rightarrow$ bābu 'entrance-door'

tैi़

KA (KA, no. 24) $\rightarrow$ pû 'mouth, opening'

(A)

KA.A.AB.BA (KA, no. 24) $\rightarrow$ imbu' tâmti 'algae’

TA TF V V V

KA.INIM.MA (KA, no. 24) $\rightarrow$ ka'inimma 'invocation'

KA

KA.KEŠDA (KA, no. 24) $\rightarrow$ kișru 'knots'

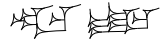


KALA.GA (KAL, no. 496) $\rightarrow$ dannu 'strong'

Uति

KÀŠ (KÀŠ, no. 384) $\rightarrow$ šīnātu 'urine'

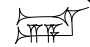

KAŠ (BI, no. 358) $\rightarrow$ šikaru 'beer'

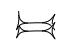

KEŠDA (EZEN, no. 271) $\rightarrow$ kașāru 'to tie, knot'

W rakāsu 'to bind, bandage body parts'

KI (KI, no. 737) $\rightarrow$ itti 'with’

陵

KI.NÁ (KI, no. 737) $\rightarrow$ mayyālu 'bed'

䧟

KÌD.KÌD.BI (AG, no. 127) $\rightarrow$ kidkidbi 'its ritual'

$\checkmark$,

KIN (KIN, no. 815) $\rightarrow$ šipru 'effect'; 'skill'

KIS

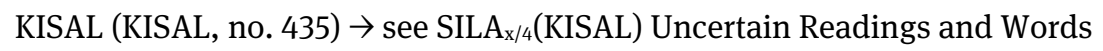
त्र

KÙ (KUG, no. 745) $\rightarrow$ ellu 'clean, holy'

侻

KÙ.BABBAR (KUG, no. 745) $\rightarrow$ kaspu 'silver'

य年

KÙ.GAN (KUG, no. 745) $\rightarrow$ lulû 'lulû-antimony'

仰

KUG.GI (KUG, no. 745) $\rightarrow$ hurāṣu 'gold'

所用家

KU.KU (KU, no. 808) $\rightarrow$ utțatu 'grain (also as a measure)'

这应

$\mathrm{KU}_{4}\left(\mathrm{KU}_{4}\right.$, no. 87) $\rightarrow$ erēbu 'enter, go down, set (for sun)'

H

KUD (TAR, no. 9) $\rightarrow$ nakāsu 'to cut'; parāsu 'to cease, stop'

此

KÚM (NE, no. 313) $\rightarrow$ ummu 'ummu-fever (lit. heat, summer)'; emmu 'hot'

然

$\mathrm{KUN}_{4}(\mathrm{I}$, no. 252) $\rightarrow$ askuppu 'threshold'

\section{等}

KUR (KUR, no. 578) $\rightarrow$ mātu 'land, earth'; šadû 'mountain'

¿s

KUR.GI'mušn (KUR, no. 578) $\rightarrow$ kurkû 'goose'

से मार्या

KUŠ (SU, no. 16) $\rightarrow$ mašku 'leather bag' ver 
KUŠ.EDIN (not in MZ) $\rightarrow$ nādu 'waterskin'

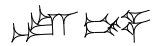

$\mathbf{L}$

LAL (LAL, no. 750) $\rightarrow$ șamādu 'to bandage'; tarāṣu 'to spread out (a cloth)'

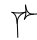

LÀL (LÀL, no. 170) $\rightarrow$ dišpu 'honey’

LA

LÀL.KUR.(RA) (LÀL, no. 170) $\rightarrow$ dišip šadê/̂̂ 'mountain honey'

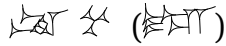

LI.DUR/TAR (LI, no. 85) $\rightarrow$ abukkatu 'abukkatu-plant'

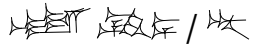

LIBIR.RA (LIBIR, no. 732) $\rightarrow$ labìru 'old'

या प्रा

LÚ (LÚ, no. 514) $\rightarrow$ amēlu ‘a man'

(1)

lúA.ZU (A, no. 839) $\rightarrow$ asû 'physician'

诰

lúA ŠGAB (AŠGAB, no. 173) $\rightarrow$ aškāpu 'leatherworker’

借

LUGAL (LUGAL, no. 266) $\rightarrow$ šarru 'king'

(1)

LÚGUD.DA (LAGAB, no. 755) $\rightarrow$ kurû 'short'

छे

LUH (LUH, no. 494) $\rightarrow$ mesû 'to wash'

trms

lúTU.RA (TU, no. 86) $\rightarrow$ marșu 'sick man'

त्र

lúTUR (TUR, no. 255) $\rightarrow$ șehru 'a chick'; 'a child'

看

LÚ.U19.LU (LÚ, no. 514) $\rightarrow$ amēlu 'a man'

证

\section{M}

20 (MAN, no. 708) $\rightarrow$ šarru 'king'

4

MAR (MAR, no. 483) $\rightarrow$ eqû 'to daub'; zarû 'scatter, spatter, sprinkle'

the 
MAŠ.DÀ (MAŠ, no. 120) $\rightarrow$ șabìtu 'gazelle'

पF

MAŠ.MAŠ (MAŠ, no. 120) $\rightarrow$ mašmašu 'mašmašu-healer'

HF

MAŠ.TAB.BA (MAŠ, no. 120) $\rightarrow$ māšu 'a twin'

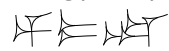

MÁŠ.TUR (MÁŠ, no. 130) $\rightarrow$ lalû 'a kid, young goat'

Vद⿱

MIN (not in MZ) $\rightarrow$ šanîš ‘alternatively’

M

MÚ (SAR, no. 541) $\rightarrow$ napāhu 'to blow; to light up, ignite'

\section{U}

MÚD (BAD, no. 113) $\rightarrow$ dāmu 'blood'

$\succ 4 \quad$ MÚD not ÚŠ, see the syllabic spelling in IGI 1: 118' (NB iii 3)

MUD (MUD, no. 137) $\rightarrow$ uppu 'tube'

布度

MUL (MUL, no. 247) $\rightarrow$ kakkabu 'a star'

FUF

MUN (MUN, no. 168) $\rightarrow$ țābtu 'salt'

逊

MUNU $_{6}$ (BÙLUG, no. 94) $\rightarrow$ buqlu 'malt'

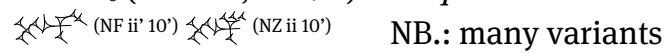

MUNUS (MUNUS, no. 883) $\rightarrow$ sinništu 'female'

L

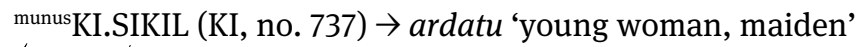

佂

munus'́.ZÚG (Ú, no. 490) $\rightarrow$ musukkatu ‘a woman in maternity’

स्य

MURUB $_{4}$ (MÚRU, no. 545) $\rightarrow$ qablu 'middle'

过位

MUŠ.GE 6 (MUŠ, no. 585) $\rightarrow$ șallāmtu 'black snake'

स्सिA th

\section{N}

NÁ (NÁ, no. 689) $\rightarrow$ șalālu 'to sleep, lie down'

2

NA (NA, no. 110) $\rightarrow$ amēlu 'a man'

$\checkmark$

NÁ (NÁ, no. 689) $\rightarrow$ itūlu 'to lie down'; nâlu 'to lie down' 蜘 
$\mathrm{NA}_{4}\left(\mathrm{NA}_{4}\right.$, no. 385) $\rightarrow$ abnu 'stone, bead'

ये

$\mathrm{NA}_{4}$ BAL (not in MZ) $\rightarrow$ aban tašrīti 'tašrītu-stone'

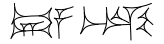

${ }^{\text {na4} A N . N A ~(A N, ~ n o . ~ 10) ~} \rightarrow$ annakku 'tin'

WF十

${ }^{n a 4}$ AN.ZAH (AN, no. 10) $\rightarrow$ anzahhu 'anzahhu-glass (bead)'

WF HF

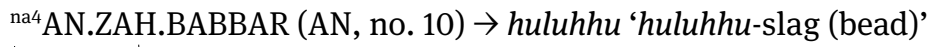

AF

${ }^{\text {na4 AN.ZAH.GE }}$ (AN, no. 10) $\rightarrow$ kutpû black frit (bead)'

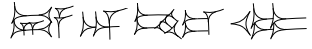

${ }^{n a}{ }^{4}$ ÁŠ.GI ${ }_{4} \cdot \mathrm{GI}_{4}(\mathrm{~A} \check{S}$, no. 548) $\rightarrow$ ašgikû 'ašgikû-stone'

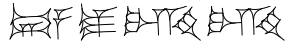

${ }^{n a}$ BAR.DÙ.E (not in MZ) $\rightarrow$ mešēltu 'mešēeltu-whetstone'

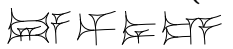

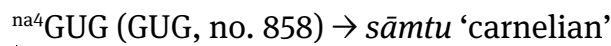

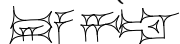

${ }^{n a}{ }^{4}$ KA.GI.NA.DAB.BA (KA, no. 24) $\rightarrow$ šadânu șābitu 'magnetite'

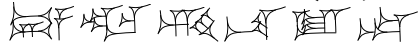

${ }^{\text {na4} M U S ̌ . G I ́ R ~(M U S ̌, ~ n o . ~ 585) ~} \rightarrow$ muššaru 'sardonyx'

UYFAMATH

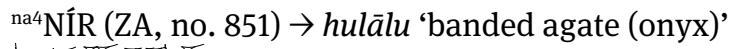

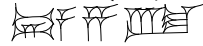

na4ŠU.MÌN (not in MZ) $\rightarrow$ šumēnu ‘šumēnu-mineral'

伊造 A

na4ŠUBA (ZA, no. 851) $\rightarrow$ šubû ‘šubû-stone’

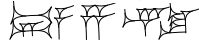

na4ZA.GÌN (ZA, no. 851) $\rightarrow$ uqnû 'blue stone, mostly lapis lazuli' क्ये से

${ }^{\text {na4ZA.GÌN.DURU }} 5$ (ZA, no. 851) $\rightarrow$ zagindurû 'zagindurû-lapis lazuli' W कि से

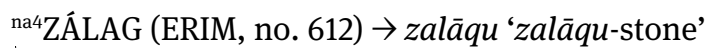

बर्ष

${ }^{n a}{ }^{4} Z U ́$ (KA, no. 24) $\rightarrow$ șurru 'flint (knife)'

伎

NAG (NAG, no. 64) $\rightarrow$ šaqû 'to give to drink'; šatû 'to drink'

锖

NAGA.SI (NAGA, no. 293) $\rightarrow$ uhūlu qarnānû 'horned uhūlu-alkali'

㑔

NAM.LÚ.U19.LU (NAM, no. 134 and 514) $\rightarrow$ amēlūtu 'human'

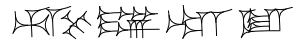


NE (NE, no. 313) $\rightarrow$ pēmtu 'charcoal'

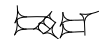

NÍG.BA (BA, no. 14) $\rightarrow$ qištu 'fee'

ए ver

NÍG.NA (NÍG, no. $859 \rightarrow$ nignakku 'incense burner'

ए广

NÍG.SILA ${ }_{11}$ GÁ (NÍG, no. 859) $\rightarrow$ lǐšu 'dough'

एक क्षा

NIGIN (NIGIN, no. 804) $\rightarrow$ lamû 'wrap up'

C广5

NIM.GÍR (NIM, no. 690) $\rightarrow$ berqu 'lighting'

सt 4 बतr

NINDA (NÍG, no. 859) $\rightarrow$ akalu 'loaf of bread'

एP

NININDU (LAGAB×NÍG, no. 801) $\rightarrow$ tinūru 'tannour oven'

(E)

NITA (UŠ, no. 381) $\rightarrow$ zikaru 'man, male'

W

NÍTA (ARAD, no. 18) $\rightarrow$ zikaru 'man, male'

tr

$\mathrm{NU}$ (NU, no. 112) $\rightarrow$ lā 'no, not, without'; ul 'no, not'

$\nLeftarrow$

NU.LUH.HA ${ }^{\text {sar }}$ (NU, no. 112) $\rightarrow$ nuhurtu 'nuhurtu-plant'

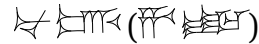

NU.NU (NU, no. 112) $\rightarrow$ țamû to spin’

$\forall$

NUMUN (NUMUN, no. 117) $\rightarrow$ zèru 'seed(s)'

$\checkmark 4$

NUNDUM (NUNDUM, no. 31) $\rightarrow$ šaptu 'lip'

(1)

NUNUZ (NUNUZ, no. 614) $\rightarrow$ pelû 'egg'

新

\section{$\mathbf{P}$}

PA (PA, no. 464) $\rightarrow$ artu 'foliage, leafs'; kappu 'eyelid' \&A

PAP (PAB, no. 92) $\rightarrow$ naphar 'total'

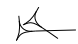

PIŠ ${ }_{10}$.'ID (KI, no. 737) $\rightarrow$ kibrītu 'kibrītu-sulphur'

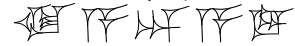


PÚ (TÚL, no. 786) $\rightarrow$ būrtu 'well'

这

$\mathbf{S}$

SA (SA, no. 172) $\rightarrow$ šer'ānu 'sinew/tendon'

SA

$\mathrm{SA}_{5}$ (DIR, no. 207) $\rightarrow$ sāmu 'red'

sm?

SAG (SAG, no. 184) $\rightarrow$ qaqqadu 'head'; rūštu 'first class, premium'

T्रT rēšu 'upper part'

SAG.DU (SAG, no. 184) $\rightarrow$ qaqqadu 'head'

许席

SAG.KI (SAG, no. 184) $\rightarrow$ nakkaptu 'head-temple'; pūtu 'forehead'

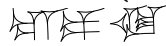

SAG.UŠ (SAG, no. 184) $\rightarrow$ kayyamānu 'regularly, again and again' प्रा

SAHAR (IŠ, no. 357) $\rightarrow$ eperu 'dust'

tor

SAHAR.KÙ.GI (not in MZ) $\rightarrow$ lēru 'lēru-paste'

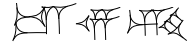

SAHAR.URUDU (IŠ, no. 357) $\rightarrow$ šuhtu 'copper patina'

BT U⿺辶万

SAR (SAR, no. 541) $\rightarrow$ galābu 'to shave'

SA

SAR.MUNU $_{6}$ (not in MZ) $\rightarrow$ bāqilu 'malster'

络

SED (SED, no. 155) $\rightarrow$ kașû 'cold'

एकिए

SI (SI, no. 181) $\rightarrow$ qarnu 'horn'

tri

SÍG (SÍG, no. 816) $\rightarrow$ šārtu 'hair’; šīpātu 'wool’

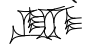

$\mathrm{SIG}_{5}\left(\mathrm{SIG}_{5}\right.$, no. 729) $\rightarrow$ damqu 'good'

या这

$\mathrm{SIG}_{7}\left(\mathrm{SIG}_{7}\right.$, no. 564) $\rightarrow$ arqu 'yellow-green'; arqūtu 'freshness'

选

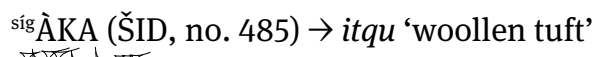

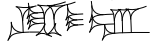

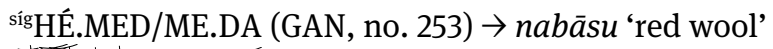

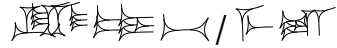


SILA (TAR, no. 9) $\rightarrow$ sūqu 'street'

放

SILAx $_{x}($ KISAL, no. 435) $\rightarrow$ see SILA $($ KISAL) Uncertain Readings and Words trit

SILA $_{4}\left(\right.$ SILA $_{4}$, no. 408) $\rightarrow$ see SILA4!(KISAL) Uncertain Readings and Words 蛋

SILA $_{11}($ ŠID, no. 485) $\rightarrow$ lâšu 'to knead'

in

SILIM (DI, no. 736) $\rightarrow$ šalāmu 'to be safe'

偻

SIM (NAM, no. 134) $\rightarrow$ napû 'to sift'

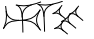

SU (SU, no. 16) $\rightarrow$ zumru 'body'

Ner

SÚD (ŠìTA, no. 139) $\rightarrow$ sâku 'to pound'

$\forall$ YK

SUHUŠ (SUHUŠ, no. 351) $\rightarrow$ šuršu 'root'

近

SUM.SIKIL (SUM, no. 292) $\rightarrow$ šamaškillu ‘šamaškillu-onion’

位

$\mathrm{SUM}^{\mathrm{sar}}(\mathrm{SUM}$, no. 292) $\rightarrow$ šūmū 'garlic'

格在

SUMUN (BAD, no. 113) $\rightarrow$ labāru 'to be(come) old, long-lasting'; labīru 'old'

$\checkmark 1$

SÚN (GUL, no. 682) $\rightarrow$ rīmtu 'wild cow'

यद

SUR (SUR, no. 151) $\rightarrow$ șahātu 'to press out, draw liquid into vessel'

ए阝

\section{$\check{\mathbf{s}}$}

ŠÀ (ŠÀ, no. 599) $\rightarrow$ libbu 'middle, midst of'; 'internal organ/heart'

类 'offshoot (of date palm)'

ŠAH (̌̌AH, no. 23) $\rightarrow$ šahû 'pig’

W

ŠE (ŠE, no. 579) $\rightarrow$ še'u 'grain'; utțatu 'grain (also as a measure)'

\&

ŠE.BAR (ŠE, no. 579) $\rightarrow$ uțtatu 'grain (also as a measure)'

SसL

ŠE.GA (ŠE, no. 579) $\rightarrow$ magāru 'to be favourable'

达许公 
ŠE.SA.A (ŠE, no. 579) $\rightarrow$ labtu 'roasted grain'

स्स Pि

$\check{S}_{10}(K U$, no. 808) $\rightarrow z \hat{u}$ 'excrement'

应

ŠEG6(.GA) (NE, no. 313) $\rightarrow$ bašālu 'to be cooked'; bašlu 'boiled'

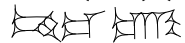

ŠEN.TUR (ŠEN, no. 17) $\rightarrow$ tangussu 'copper tangussu-kettle'

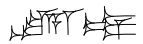

ŠÉŠ (ŠÉŠ, no. 821) $\rightarrow$ pašāšu 'to smear, anoint'

ST

ŠEŠ (ŠEŠ, no. 535) $\rightarrow$ annû 'this, these'

年

ŠID (ŠID, no. 485) $\rightarrow$ manû 'to recite'

피

ŠIKA (LA, no. 89) $\rightarrow$ hașbu 'potsherd, sherd, peel, flake'

N

šmBAL (BAL, no. 5) $\rightarrow$ ballukku 'ballukku-aromatic'

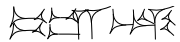

ŠIM.BI.KÙ.GI (ŠIM, no. 362) $\rightarrow$ šīpu ‘šīpu-paste’

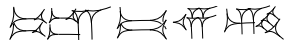

ŠIM.BI.ZI.DA (ŠIM, no. 362) $\rightarrow$ šimbizidû ‘šimbizidû-galena'

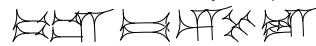

šmBULUH (HAL, no. 3) $\rightarrow$ baluhhu 'baluhhu-aromatic'

ty

šm GAM.MA (GAM, no. 576) $\rightarrow$ șumlalû 'ṣumlalû-aromatic plant'

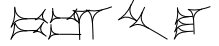

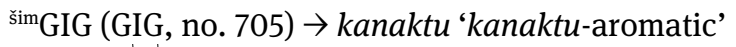

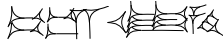

šmGIIR (GÍR, no. 6) $\rightarrow$ asu 'asu-aromatic'

परT

šmGÚR.GÚR (GAM, no. 576) $\rightarrow$ kukru 'kukru-aromatic'

政 出

šmHAB (LAGAB, no. 755) $\rightarrow$ țūru 'țūru-plant'

का

šmLI (LI, no. 85) $\rightarrow$ burāšu 'juniper'

पर्या

šmMAN.DU (MAN, no. 708) $\rightarrow$ suādu 'suādu-aromatic'

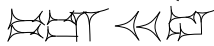

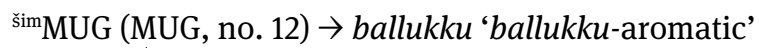
प⿺辶⿻丷木斤

šmŠE.LI (ŠE, no. 579) $\rightarrow$ kikkirânu 'kikkirânu-aromatic'

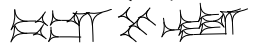


šmSES (ŠEŠ, no. 535) $\rightarrow$ murru 'bitter-plant' Sथि

ŠU (ŠU, no. 567) $\rightarrow$ qātu 'hand'

定

ŠÚ (ŠÚ, no. 869) $\rightarrow$ kiššatu 'world'

K

ŠU.GIDIM.MA (ŠU, no. 567) $\rightarrow$ šugidimmakku 'hand of a ghost'

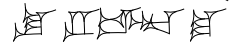

ŠU.LÚ.ZABAR (not in MZ) $\rightarrow$ mušālu 'bronze palette, mirror' 每

ŠU.SAR (ŠU, no. 576) $\rightarrow$ pitiltu 'string'

店结

ŠU.SI (ŠU, no. 567) $\rightarrow$ ubānu 'finger'

许

ŠU.TI (ŠU, no. 567) $\rightarrow$ leqû 'to take, accept, + ventive to bring' U

ŠUB (RU, no. 111) $\rightarrow$ nadû 'to put, apply...'

近

ŠURUN $\left(\mathrm{U}_{8}\right.$, no. 766) $\rightarrow$ kabūtu 'dung'

䧟后

$\mathbf{T}$

TAB (TAB, no. 209) $\rightarrow$ tepû 'to apply (drugs), add (ingredients)'

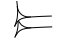

TE (TE, no. 589) $\rightarrow$ tehû 'to be(come) near to, approach'

还

TI (TI, no. 118) $\rightarrow$ balāṭ 'to live, heal, recover'; bulțu 'life' $\checkmark \sqrt{A}$

TI.LA (TI, no. 118) $\rightarrow$ balātu 'to live, heal, recover'; balțu 'healthy' MAV V

TI (TI, no. 118) $\rightarrow$ leqû 'to take, accept, + ventive to bring' Uाब

$\mathrm{TI}_{8}{ }^{\text {mušen }}(\hat{\mathrm{A}}$, no. 560) $\rightarrow$ erû 'erû-eagle'

WA

$\mathrm{TU}_{5}($ ŠU, no. 567) $\rightarrow$ ramāku 'to bathe, soak'

年

$\mathrm{TU}_{6}\left(\mathrm{TU}_{6}\right.$, no. 26) $\rightarrow$ tû 'incantation'

速

$\mathrm{TU}_{6 .}$ ÉN $\left(\mathrm{TU}_{6}\right.$, no. $\left.^{26}\right) \rightarrow$ tê šipti

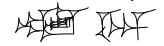


TU6.ÉN.É.NU.RU $\rightarrow$ Uncertain Readings and Words

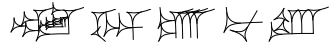

TÚG (TÚG, no. 809) $\rightarrow$ șubātu 'cloth'

㹣

túš̌̀̀.HA (TÚG, no. 809) $\rightarrow$ šahhû ‘šahhû-cloth’

迹䣄前

TUK (TUK, no. 827) $\rightarrow$ rašû 'to acquire, to develop a disease'

莼

TUmušen (TU, no. 86) $\rightarrow$ summatu 'pigeon'

TAT

TÙR (TÙR, no. 146) $\rightarrow$ tarbașu 'animal stall'

AMPTY

TUR (TUR, no. 255) $\rightarrow$ șehru 'small, young'

W

\section{U}

Ú (Ú, no. 490) $\rightarrow$ šammu 'plant, drug'

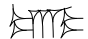

Ú.BABBAR (Ú, no. 490) $\rightarrow$ šammu peșû 'white plant'

पme so

Ú.DILI (AŠ, no. 1) $\rightarrow$ šammu èdu 'single plant'

角在

Ù.TU (Ù, no. 731) $\rightarrow$ alādu 'to give birth'

या

$\mathrm{U}_{5 .} \mathrm{ARGAB}^{\text {mušen }}\left(\mathrm{U}_{5}\right.$, nos. 133 and 859$) \rightarrow$ rikibti arkabi 'bat guano'

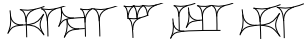

$\mathrm{U}_{8}\left(\mathrm{U}_{8}\right.$, no. 766$) \rightarrow$ lahru 'ewe'

啮

UD (UD, no. 596) $\rightarrow$ ūmu 'day'; enūma 'when'

\&

UD.1/3/9/14/15/20/21.KAM/KÁM/KAM ${ }^{\star} \rightarrow$ Uncertain Readings and Words

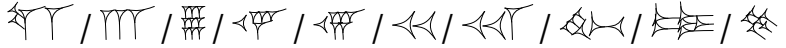

UD.DA (UD, no. 596) $\rightarrow$ șētu 'sun heat'

4 更

$\mathrm{UD}_{5}$ (ÙZ, no 203) $\rightarrow$ enzu 'goat'

Tm

UDU (LU, no. 812) $\rightarrow$ immeru 'sheep'

田

UDU.NÍTA (LU, no. 812) $\rightarrow$ immeru 'male sheep'

严 
UDU.SISKUR (SISKUR, no. 696) $\rightarrow$ niqû 'offering'

证

úEME.UR.GI 7 (not in MZ) $\rightarrow$ lišān kalbi 'dog's tongue plant'

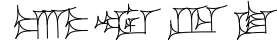

úESI (KAL, no. 496) $\rightarrow$ ušû 'ušû-plant'

का

"GAMUN (DIN, no. 119) $\rightarrow$ kamūnu ‘cumin'

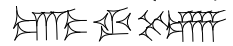

$\mathrm{UGA}^{\text {mušen }}(\mathrm{UGA}$, no. 490) $\rightarrow$ āribu 'raven'

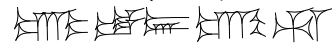

"GEŠTIN.KA 5 .A (not in MZ) $\rightarrow$ karān šēlebi 'fox-vine'

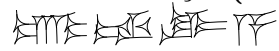

UGU (UGU, no. 663) $\rightarrow$ eli 'on, over, above’

muhhu 'cranium, brain, top'; 'top, upper part, surface'

úGÚR.UŠ (not in MZ) $\rightarrow$ šarmadu 'šarmadu-plant'

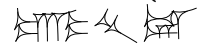

ÚH (ÚH, no. 611) $\rightarrow$ ru'tu 'spittle'

妨出个

ÚH-díD (ÚH, no. 611) $\rightarrow$ ru'tītu 'ru'tītu-sulphur (lit. river spittle)'

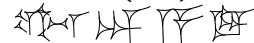

úHA (HA, no. 856) $\rightarrow$ urânu 'urânu-plant'

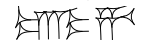

úHAR.HAR (HAR, no. 644) $\rightarrow$ hašû 'hašû-thyme'

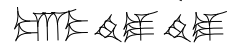

úIGI-lim (IGI, no. 724) $\rightarrow$ imhur-lìm 'imhur-lìm-plant'

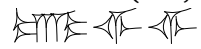

'IGI-NIŠ (IGI, no. 724) $\rightarrow$ imhur-ešrā 'imhur-ešrāa-plant'

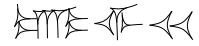

"IN.NU.UŠ (IN, no. 261) $\rightarrow$ maštakal 'maštakal-plant'

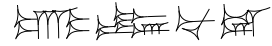

'IN 6 .ÚŠ (AŠ, no. 1) $\rightarrow$ maštakal 'maštakal-plant'

trmers

'KI. 'dŠKUR (KI, no. 737) $\rightarrow$ qudru 'qudru-plant'

到在证 UF

'KUR.KUR (KUR, no. 578) $\rightarrow$ atā'išu 'atā'išu-plant'

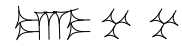

'KUR.RA (Ú, no. 490) $\rightarrow$ nīnû 'nīnû-mint'

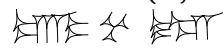

"KUR4.GI.RIN.NA (not in MZ) $\rightarrow$ kurkānû 'kurkānû-plant'

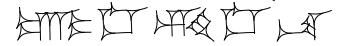
(LAGAB in ND ii 41 )

UL (UL, no. 698) $\rightarrow$ kakkabu 'a star' 原 
úMAŠ.TAB.BA (MAŠ, no. 120) $\rightarrow m \bar{a} \check{s}(t) u$ ' $m \bar{a} \check{s}(t) u$-twin plant'

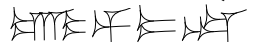

UMBIN (UMBIN, no. 160) $\rightarrow$ șupru '(toe-) nail'

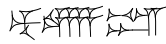

UM.ME.A (UM, no. 248) $\rightarrow$ ummânu 'wise man'

铈原商

úNAGA.SI (NAGA, no. 293) $\rightarrow$ uhūlu qarnānû 'horned uhūlu-alkali'

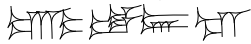

úNÍG.GIDRU (PA, no. 464) $\rightarrow$ hațti rē'̂̀ 'shepherd's-staff plant'

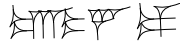

UR.BI (UR, no. 828) $\rightarrow$ ištēniš 'together'

DAY $\&$

ÙR (ÙR, no. 411) $\rightarrow$ ūru 'roof'

mar

$\mathrm{UR} \mathrm{GI}_{7}(\mathrm{UR}$, no. 828) $\rightarrow$ kalbu 'dog'

位

UR.MAH (UR, no. 828) $\rightarrow$ nēšu 'lion'

DE

URUDU (URUDU, no. 230) $\rightarrow$ erû 'erû-copper'

UT

'SIKIL (EL, no. 899) $\rightarrow$ sikillu 'sikillu-plant'

出在界

ÚŠ (BAD, no. 113) $\rightarrow$ sekēru 'to heat'

$\downarrow 4$

úUKUŠ.LAGAB (HÚL, no. 877) $\rightarrow$ errû 'errû-colocynth'

पा לै।

úUR.TÁL.TÁL (UR, no. 828) $\rightarrow$ uzun lalî 'young goat's-ear plant'

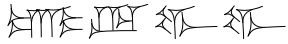

úZA.BA.LAM (ZA, no. 851) $\rightarrow$ supālu ‘supālu-juniper'

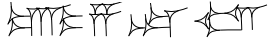

úZA.GÌN.NA (ZA, no. 851) $\rightarrow$ uqnâtu 'uqnâtu-blue-plant'

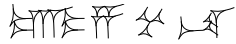

UZU (UZU, no. 311) $\rightarrow$ šēru 'flesh'

tor

uzuzí (ZÍ, no. 259) $\rightarrow$ martu 'gall bladder'

at

Z

ZÀ.HI.LI(.A $\left.{ }^{\text {sar }}\right)(Z A G$, no. 540) $\rightarrow$ sahlû 'sahlû-plant'

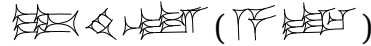


ZA.NA (ZA, no. 851) $\rightarrow$ passu 'collyrium stick (lit. game disk)' 落头

ZABAR (UD, no. 596) $\rightarrow$ siparru 'bronze' \& 5 \&

ZAG (ZAG, no. 540) $\rightarrow$ imittu 'right side' 1

ZALAG.GA (not in MZ) $\rightarrow$ namāru 'to be(come) bright, shine'

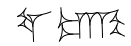

ZI (ZI, no. 140) $\rightarrow$ nasāhu 'to tear out, remove'; tebû 'to rise, throb' भfoke tìbu 'rising (of wind)'

ZÍ (ZÍ, no. 259) $\rightarrow$ martu 'bile'

है।

Zİ (ŠÈ, no. 810) $\rightarrow$ qēmu 'flour, powder' 这

Zİ.DUB.DUB.BU (ŠÈ, no. 810) $\rightarrow$ zidubdubbû 'a heap of flour'

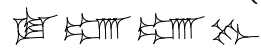

Zİ.KUM (ŠÈ, no. 810) $\rightarrow$ isqūqu 'coarse flour'

造全

ZÚ.LUM.MA (KA, no. 24) $\rightarrow$ suluppu 'date’ 计 